\title{
ASYMPTOTIC BEHAVIOR OF BV FUNCTIONS AND SETS OF FINITE PERIMETER IN METRIC MEASURE SPACES
}

\author{
SYLVESTER ERIKSSON-BIQUE, JAMES T. GILL, \\ PANU LAHTI, AND NAGESWARI SHANMUGALINGAM
}

\begin{abstract}
In this paper, we study the asymptotic behavior of BV functions in complete metric measure spaces equipped with a doubling measure supporting a 1-Poincaré inequality. We show that at almost every point $x$ outside the Cantor and jump parts of a BV function, the asymptotic limit of the function is a Lipschitz continuous function of least gradient on a tangent space to the metric space based at $x$. We also show that, at co-dimension 1 Hausdorff measure almost every measure-theoretic boundary point of a set $E$ of finite perimeter, there is an asymptotic limit set $(E)_{\infty}$ corresponding to the asymptotic expansion of $E$ and that every such asymptotic limit $(E)_{\infty}$ is a quasiminimal set of finite perimeter. We also show that the perimeter measure of $(E)_{\infty}$ is Ahlfors co-dimension 1 regular.
\end{abstract}

Key words and phrases: Bounded variation, finite perimeter, asymptotic limit, doubling measure, Poincaré inequality, least gradient function.

Mathematics Subject Classification (2010): 30L99, 26A45, 31E05, 43A85.

\section{INTRODUCTION}

The classical notion of differentiability for a function $f$ on a subset of Euclidean space $\Omega \subset \mathbb{R}^{n}$ at a point $x \in \Omega$ is that the graph of $f$ should, at $(x, f(x)) \in \mathbb{R}^{n} \times \mathbb{R}$, asymptotically approach an $n$-dimensional hyperplane in $\mathbb{R}^{n+1}$ as we zoom in. In other words, the function $f$ behaves asymptotically like an affine function. This notion has been extended to mappings between domains in Riemannian manifolds in the study of differential geometry.

The seminal work of Cheeger [11] extended the above notion of affine approximation to the realm of metric measure spaces, with generalized linear functions defined on measured Gromov-Hausdorff tangent spaces playing the role of affine functions. It was shown there that, if the space is complete, the measure is doubling, and the space supports a $p$-Poincaré inequality for some $1 \leq p<\infty$, then every Lipschitz function $f$ on the metric space is asymptotically generalized linear at almost every point $x$ in the space. More specifically, we have the following: let $X_{\infty}$ be obtained as a pointed measured Gromov-Hausdorff limit of scaled versions $\left(X_{n}, d_{n}, x, \mu_{n}\right)$ of the metric measure space

The first author was supported by NSF grant \#DMS-1704215. The third author was partially supported by the Finnish Cultural Foundation. The fourth author was partially supported by the NSF grant \#DMS-1500440 (U.S.). The motivation for this research came from a recommendation by Tatiana Toro to study asymptotic behavior of sets of finite perimeter, during the fourth author's visit to University of Washington at Seattle in 2010; the authors wish to thank her for the valuable comments that motivated this research. Part of the research was done during the visit of the second and third authors to University of Cincinnati, and during the period the first, third, and fourth authors were visiting Linköping University supported by a departmental grant from the Wallenberg Foundation; they wish to thank those institutions for their kind hospitality. We also wish to thank Tapio Rajala for valuable discussions that lead to Example 6.13. We are grateful to the referee, whose kind comments helped improve the exposition of the paper. 
$(X, d, \mu)$ with $x \in X$. In considering corresponding scaled versions $f_{n}: X_{n} \rightarrow \mathbb{R}$ of $f: X \rightarrow \mathbb{R}$, where

$$
f_{n}(y):=\frac{f(y)-f(x)}{r_{n}}
$$

with $\left\{r_{n}\right\}_{n \in \mathbb{N}}$ a sequence of positive numbers decreasing to zero which form the scales associated with the metric $d_{n}:=r_{n}^{-1} d$ in the Gromov-Hausdorff limit, the sequence of functions $f_{n}$ converges to a limit function $f_{\infty}: X_{\infty} \rightarrow \mathbb{R}$ (after passing to a subsequence if necessary). Cheeger proved that this asymptotic limit function $f_{\infty}$ is a generalized linear function on $X_{\infty}$. Here by a generalized linear function, the paper [11] means a function that is $p$-harmonic on $X_{\infty}$ with a constant function as its minimal $p$-weak upper gradient.

In this paper, we extend the study of asymptotic behavior of Lipschitz functions in [11] to functions of bounded variation in complete metric measure spaces equipped with a doubling measure supporting a 1-Poincaré inequality. The following theorems give a summary of the principal results of this paper; the precise versions can be found in the statements of the corresponding theorems in Sections $4-6$.

Theorem A (Theorems 4.8 and 4.9). Suppose that the measure $\mu$ on the complete metric space $(X, d)$ is doubling and supports a 1-Poincaré inequality. Let $u$ be a function of bounded variation on $X$. For $\mu$-a.e. $x \in X$ and any tangent space $\left(X_{\infty}, d_{\infty}, x_{\infty}, \mu_{\infty}\right)$ of $X$ based at $x$, any limit function $u_{\infty}$ as described above is 1-harmonic (also known as function of least gradient) and has quasi-constant minimal 1-weak upper gradient.

The definitions of doubling and Poincaré inequality can be found in (2.1) and (2.4) below.

The most fundamental BV functions are characteristic functions of sets of finite perimeter. For these functions, the most interesting behavior happens solely at their jump points. Here the study of asymptotics is different, see e.g. [12, Theorem 5.13] in the Euclidean setting. Similarly, for general BV functions $u$, the approach of scaling the function as described above works well when considering points in $X$ that asymptotically see neither the Cantor nor the jump parts of the variation measure $\|D u\|$ of $u$, but it is not helpful in the study of asymptotic behavior of $u$ at points in its jump set $S_{u}$. Instead, an approach based on weak* limits of measures, which can also be used to define the limit function $u_{\infty}$ as in Theorem 5.5, is more in line with studying the behavior of $u$ at points in the jump set $S_{u}$ of $u$ and gives an alternative approach to Theorem A. This measure-theoretic approach is applied to characteristic functions of sets of finite perimeter in Sections 5 and 6 and the main conclusions are described in Theorems B and $\mathrm{C}$ below.

Theorem B (Theorem 5.5). Suppose that the measure $\mu$ on the complete metric space $(X, d)$ is doubling and supports a 1-Poincaré inequality. Let $E \subset X$ be of finite perimeter $P(E, \cdot)$. Then for $P(E, \cdot)$-a.e. point, appropriately scaled versions of $P(E, \cdot)$ converge to a measure on $X_{\infty}$ that is comparable to the co-dimension 1 Hausdorff measure restricted to the measure-theoretic boundary of a set $(E)_{\infty}$ of locally finite perimeter in $X_{\infty}$.

In $\mathbb{R}^{n}$, the corresponding limits are not just $(n-1)$-dimensional, but are hyperplanes that are boundaries of sets whose characteristic functions are functions of least gradient, that is, of minimal boundary surface. In the metric setting, we obtain an analogue with quasiminimal sets playing the role of hyperplanes and minimal boundary surfaces.

Theorem C (Theorem 6.3). Suppose that the measure $\mu$ on the complete metric space $(X, d)$ is doubling and supports a 1-Poincaré inequality. Let $E \subset X$ be of finite perimeter. Then, with respect to the co-dimension 1 Hausdorff measure, almost every point $x$ on the measure-theoretic boundary 
of $E$ satisfies the following properties: fixing a (pointed) tangent space $\left(X_{\infty}, d_{\infty}, x_{\infty}, \mu_{\infty}\right)$ arising as a Gromov-Hausdorff limit of the scaled sequence $\left(X_{n}, d_{n}, x, \mu_{n}\right)$, and by passing to a subsequence if necessary we obtain that

- the sequence of measures $\chi_{E} d \mu_{n}$ on $X_{n}$ converges weakly* to a measure $\mu_{\infty}^{E}$ on $X_{\infty}$,

- this limit measure is absolutely continuous with respect to $\mu_{\infty}$,

- there is a set $(E)_{\infty} \subset X_{\infty}$ of finite perimeter such that $d \mu_{\infty}^{E}=\chi_{(E)_{\infty}} d \mu_{\infty}$,

- the set $(E)_{\infty}$ is of quasiminimal boundary surface (see Definition 6.2 or [25]), and

- the measure described in Theorem $B$ is supported on the boundary of $(E)_{\infty}$, and is comparable to the perimeter measure $P\left((E)_{\infty}, \cdot\right)$ of $(E)_{\infty}$.

Thus, beginning with extensions of the Cheeger's Rademacher theorem for doubling metric spaces with a Poincaré inequality to the functions of bounded variation, we recover important aspects of the classical theory of the boundaries of finite perimeter sets in $\mathbb{R}^{n}$.

The class of BV functions considered here is based on the notion first proposed by Miranda Jr. [33], and was further developed in [1, 5, 2]. The corresponding notion of a function of least gradient was studied in $[25,18,28]$. Just as [11] related asymptotic limits of Lipschitz functions to generalized linear functions (which are a priori $p$-harmonic for the indices $p>1$ for which $X$ supports a $p$-Poincaré inequality), we relate asymptotic limits of BV functions to functions of least gradient when the point of asymptoticity does not lie in the set where the jump and Cantor parts of the variation measure live. Additionally, at almost every point with respect to the co-dimension 1 Hausdorff measure in the measure-theoretic boundary of the set of finite perimeter, we relate the asymptotic limit of that set to sets of finite perimeter that have a quasiminimal boundary surface as in $[25]$.

In the setting of Heisenberg groups (perhaps the simplest non-Riemannian example of the type of metric measure spaces studied here), more is known of the asymptotic behavior of BV functions; the key papers to study this setting are those of Magnani [32], Franchi, Serapioni and Serra-Cassano [13], and Ambrosio, Ghezzi and Magnani [4]. It is shown in [13, Theorem 4.1] that asymptotic limits of sets of finite perimeter in a Heisenberg group, based at a reduced boundary point of that set, are Euclidean (vertical) half-spaces with the boundary plane parallel to the non-horizontal direction. Studies of asymptotic limits of sets of finite perimeter in more general step-2 Carnot groups can be found in [14], and for more general Carnot groups in [15]. While the Heisenberg groups are topologically Euclidean, there are more sets of finite perimeter in the Heisenberg sense than in the Euclidean sense, see [13, Proposition 2.15]. The papers [13, 14, 15] rely on the group structure on the Carnot groups, and so they do not address the case of more general Carnot-Carathéodory spaces. Carnot-Carathéodory spaces can be realized as Riemannian manifolds equipped with a vector field that satisfies a Hörmander-type condition, called the horizontal vector field, see [7] for more on these spaces.

Carnot-Carathéodory spaces are (locally) doubling metric measure spaces supporting a 1-Poincaré inequality, and hence the results of the present paper also apply there. Note that tangent spaces of Carnot-Carathéodory spaces are topological groups equipped with dilation operations, and if the tangent space is based at a regular point of the Carnot-Carathéodory space, then it is a nilpotent group equipped with a dilation, see [34, 7, 30]. Under further assumptions on the CarnotCarathéodory space (which lead to knowing that the tangent spaces are all Carnot groups), a similar asymptoticity study is undertaken in [4]. We point out here that the results in the current paper are applicable to all Carnot-Carathéodory spaces of topological dimension at least 2.

If $\nu$ is a Radon measure on $X$ and $x \in X$, then for almost every $r>0$ we know that $\nu(\bar{B}(x, r) \backslash$ $B(x, r))=0$. If $X$ is a geodesic space and $\mu$ is a doubling measure, then $\mu(\bar{B}(x, r) \backslash B(x, r))=0$ 
for each $r>0$ and $x \in X$, see [9, Corollary 2.2]. In this paper, we will assume that $X$ is geodesic in order to simplify many of the proofs (by avoiding the discussion of having to slightly adjust the radius $r$ in order to ensure that $\mu(\bar{B}(x, r) \backslash B(x, r))=0)$, but our results hold also in spaces that are not geodesic by an easy (but notationally cumbersome) modification discussed in Section 2 below.

The structure of this paper is as follows. In Section 2 we give the basic definitions necessary for the study of sets of finite perimeter and functions of bounded variation on metric measure spaces. In Section 3 we discuss pointed measured Gromov-Hausdorff limits. In Section 4 we show the results stated above regarding that asymptotic limits of BV functions converge to a function of least gradient (1-harmonic) in the tangent space, see Theorem 4.9. In Section 5 we discuss asymptotic limits of a set of finite perimeter, and show that for co-dimension 1 almost every point on the measure-theoretic boundary of that set we have a tangential behavior of the set; more specifically, there is a Gromov-Hausdorff type limit $(E)_{\infty}$ of the set $E$ at such a point, and this limit is a set of (locally) finite perimeter; this is the content of Theorem 5.5. We also verify certain geometric structural regularity of these limit sets, see Theorem 5.4. The final section of this paper is devoted to the discussion on asymptotic minimality for sets of finite perimeter. In Theorem 6.3 we show that these limit sets $(E)_{\infty}$ are sets of quasiminimal boundary surfaces.

\section{Notation AND DEFinitions}

Here we lay out the main definitions and assumptions for this paper. Much of the terminology will be similar to that used in $[1,5,33]$.

We assume that $(X, d, \mu)$ is a complete metric measure space with diam $X>0$, that is, $X$ consists of at least two points. We use the notation $B(x, r)$ for the open ball centered at $x \in X$ and of radius $r>0$. If we wish to be specific that the ball is in the metric space $X$, we write $B_{X}(x, r)$. Given a ball $B=B(x, r)$, we sometimes denote $\operatorname{rad} B:=r$; note that in metric spaces, a ball (as a set) does not necessarily have a unique center and radius, but we understand these to be prescribed for all balls that we consider. We will always assume that $\mu$ is doubling: there is a constant $C_{d} \geq 1$ such that for all $x \in X$ and $r>0$,

$$
0<\mu(B(x, 2 r)) \leq C_{d} \mu(B(x, r))<\infty .
$$

By iterating the doubling condition, we obtain for any $x \in X$ and any $y \in B(x, R)$ with $0<r \leq$ $R<\infty$ that

$$
\frac{\mu(B(y, r))}{\mu(B(x, R))} \geq \frac{1}{C_{d}^{2}}\left(\frac{r}{R}\right)^{Q}
$$

where $Q>1$ only depends on the doubling constant $C_{d}$.

When a property holds outside a set of $\mu$-measure zero, we say that it holds for $\mu$-a.e. $x \in X$. As complete doubling metric spaces are proper, every closed and bounded set is compact, see for instance [21, Lemma 4.1.14]. Given an open set $W \subset X$, we take $\operatorname{Lip}_{\text {loc }}(W)$ to be the space of functions on $W$ that are Lipschitz on every closed and bounded subset of $W$, and $L_{\text {loc }}^{1}(W)$ to be the space of functions integrable with respect to $\mu$ on every closed and bounded subset of $W$. We say that a sequence of functions $f_{k}$ converges to a function $f$ in $L_{\mathrm{loc}}^{1}(W)$ if for every closed and bounded subset $K$ of $W$ we have that $\lim _{k \rightarrow \infty} \int_{K}\left|f_{k}-f\right| d \mu=0$. It is not difficult to see that $\operatorname{Lip}_{\text {loc }}(W)$ is a dense subclass of $L_{\text {loc }}^{1}(W)$.

Given a rectifiable curve $\gamma:[0,1] \rightarrow X$, we define the length of $\gamma$ to be

$$
\ell(\gamma):=\sup \sum_{i} d\left(\gamma\left(t_{i}\right), \gamma\left(t_{i+1}\right)\right)
$$


where the supremum is taken over all finite partitions $\left\{t_{i}\right\}$ of $[0,1]$. We will always assume that $X$ is a geodesic space: for all $x, y$ in $X$,

$$
d(x, y)=\min \ell(\gamma)
$$

where the minimum is taken over all curves $\gamma$ joining $x$ to $y$ and is achieved.

Given a function $u: X \rightarrow \mathbb{R}$, an upper gradient $g$ of $u$ is a nonnegative Borel function such that for every $x, y \in X$ and every rectifiable curve $\gamma$ containing $x$ and $y$, we have the inequality

$$
|u(x)-u(y)| \leq \int_{\gamma} g d s,
$$

where $d s$ is arc length (see [21, Sections 6.2 and 6.3] for more information and standard results about upper gradients).

We say that a family of rectifiable curves $\Gamma$ is of zero $p$-modulus, for $1 \leq p<\infty$, if there is a nonnegative Borel function $\rho \in L^{p}(X)$ such that for all curves $\gamma \in \Gamma$, the curve integral $\int_{\gamma} \rho d s$ is infinite. If $g$ is a nonnegative $\mu$-measurable function on $X$ and (2.3) holds for all curves apart from a family with zero $p$-modulus, we say that $g$ is a $p$-weak upper gradient of $u$. It is known that if a function $u$ on $X$ has an upper gradient in $L^{p}(X)$, then there exists a minimal $p$-weak upper gradient of $u$, denoted by $g_{u}$, satisfying $g_{u} \leq g$ a.e. for any $p$-weak upper gradient $g \in L^{p}(X)$ of $u$, see [8, Theorem 2.25].

We always assume that the space $X$ supports a 1-Poincaré inequality. We say that $X$ supports a $p$-Poincaré inequality, for $1 \leq p<\infty$, if there is a constant $C_{P}>0$ so that for every $u \in \operatorname{Lip}_{\text {loc }}(X)$, every upper gradient $g$ of $u$, and every ball $B=B(x, r)$,

$$
f_{B}\left|u-u_{B}\right| d \mu \leq C_{P} r\left(f_{B} g^{p} d \mu\right)^{1 / p}
$$

where

$$
u_{B}:=f_{B} u d \mu:=\frac{1}{\mu(B)} \int_{B} u d \mu .
$$

We will sometimes suppress the "1-" when discussing the inequality. We will denote by $C \geq 1$ a generic constant that only depends on the doubling and Poincaré constants $C_{d}, C_{P}$, and whose precise value may change even in the same line.

We now wish to discuss functions of bounded variation and sets of finite perimeter in the metric space $(X, d, \mu)$. The definitions are quite different than those typically used for $X=\mathbb{R}^{n}$; see [33] for discussion relating these to the classical definitions. Many results from [33] and [1] will be used (and cited) in what follows. For $u \in \operatorname{Lip}_{\text {loc }}(X)$, we define

$$
\operatorname{lip} u(x):=\liminf _{r \rightarrow 0} \frac{\sup _{y \in B(x, r)}|u(y)-u(x)|}{r},
$$

often known as the lower Lipschitz constant of $u$ at $x$. It is well known that lip $u$ is an upper gradient of $u$ (see [33, Section 2], for example). We also define

$$
\operatorname{Lip} u(x):=\limsup _{r \rightarrow 0} \frac{\sup _{y \in B(x, r)}|u(y)-u(x)|}{r},
$$

the upper Lipschitz constant of $u$ at $x$. 
Since $\operatorname{Lip}_{\text {loc }}(X)$ is dense in $L_{\text {loc }}^{1}(X)$, we define the total variation of $u \in L_{\text {loc }}^{1}(X)$ on an open set $W \subset X$ as

$$
V(u, W):=\inf \left\{\liminf _{i \rightarrow \infty} \int_{W} \operatorname{lip} u_{i} d \mu: u_{i} \in \operatorname{Lip}(W), u_{i} \rightarrow u \text { in } L_{\mathrm{loc}}^{1}(W)\right\} .
$$

A function $u \in L_{\mathrm{loc}}^{1}(X)$ is said to be of locally bounded variation if $V(u, W)$ is finite for all bounded open $W \subset X$. A function $u$ is said to be of bounded variation if $V(u, X)$ is finite. Let $B V(X)$ denote the set of functions of bounded variation. For an arbitrary set $A \subset X$, we define

$$
V(u, A):=\inf \{V(u, W): A \subset W, W \subset X \text { is open }\} .
$$

If $V(u, X)<\infty$, then $V(u, \cdot)$ is a Radon measure on $X$ by [33, Theorem 3.4], called the variation measure. In much of current literature on BV functions in metric setting, $V(u, A)$ is also denoted $\|D u\|(A)$. In a significant part of the current literature on BV functions in metric spaces a slightly different notion of $V(u, W)$ is used, where instead of infimum over $\int_{W}$ lip $u_{i} d \mu$ the infimum of the integrals $\int_{W} g_{u_{i}} d \mu$ is considered, where $g_{u_{i}}$ is the minimal 1-weak upper gradient of $u_{i}$, see for example [25]. It follows from [2] that these notions all give the same BV class as well as the same BV energy $V(u, W)$ for open sets $W$ (and hence all Borel sets). Thus we can equivalently define

$$
V(u, W):=\inf \left\{\liminf _{i \rightarrow \infty} \int_{W} g_{u_{i}} d \mu: u_{i} \in \operatorname{Lip}_{\mathrm{loc}}(W), u_{i} \rightarrow u \text { in } L^{1}(W)\right\} .
$$

Let $E \subset X$ and let $\chi_{E}$ denote the characteristic function of $E$. If $\chi_{E}$ is of locally bounded variation we say that $E$ is of locally finite perimeter and if $\chi_{E}$ is of bounded variation, we say that $E$ is of finite perimeter. We use $P(E, \cdot):=V\left(\chi_{E}, \cdot\right)$ for the perimeter measure.

The following coarea formula is proven in [33, Proposition 4.2]: if $u \in B V(X)$ and $W \subset X$ is a Borel set, then

$$
V(u, W)=\int_{-\infty}^{\infty} P(\{u>t\}, W) d t .
$$

Applying the 1-Poincaré inequality to approximating functions, we get for any $\mu$-measurable set $E \subset X$ and any ball $B=B(x, r)$ the relative isoperimetric inequality

$$
\min \{\mu(B(x, r) \cap E), \mu(B(x, r) \backslash E)\} \leq 2 C_{P} r P(E, B(x, r)),
$$

see e.g. [27, Theorem 3.3].

The 1-Poincaré inequality implies the so-called Sobolev-Poincaré inequality, see e.g. [8, Theorem 4.21], from which we get the following BV version: for every ball $B(x, r)$ and every $u \in L_{\text {loc }}^{1}(X)$, we have

$$
\left(f_{B(x, r)}\left|u-u_{B(x, r)}\right|^{Q /(Q-1)} d \mu\right)^{(Q-1) / Q} \leq C r \frac{V(u, B(x, 2 r))}{\mu(B(x, 2 r))}
$$

where $Q$ is the exponent from (2.2).

Moreover, we have the following Poincaré inequality for functions vanishing outside a ball. For any ball $B(x, r)$ with $0<r<\frac{1}{4} \operatorname{diam} X$ and any $u \in L^{1}(B(x, r))$ with compact support in $B(x, r)$, we have

$$
\int_{B(x, r)}|u| d \mu \leq C r V(u, B(x, r))
$$

this again follows by applying the analogous inequality for Lipschitz functions (see [8, Theorem 4.21, Theorem 5.51]) to an approximating sequence. 
For a set $E \subset X$, the measure-theoretic boundary is defined as the set of points of positive upper density for $E$ and $X \backslash E$ :

$$
\partial^{*} E:=\left\{x \in X: \limsup _{r \rightarrow 0} \frac{\mu(B(x, r) \cap E)}{\mu(B(x, r))}>0 \quad \text { and } \quad \limsup _{r \rightarrow 0} \frac{\mu(B(x, r) \backslash E)}{\mu(B(x, r))}>0\right\} .
$$

We will also be interested in co-dimension 1 Hausdorff measures on $X$. Recall that $\mu$ is Ahlfors $s$ regular for $s>0$ if there is some constant $C_{A} \geq 1$ such that whenever $x \in X$ and $0<r<\frac{1}{2} \operatorname{diam} X$,

$$
\frac{r^{s}}{C_{A}} \leq \mu(B(x, r)) \leq C_{A} r^{s} .
$$

If $\mu$ is Ahlfors $s$-regular, then the co-dimension 1 Hausdorff measure defined below is just (comparable to) the $(s-1)$-dimensional Hausdorff measure. We do not wish to always assume Ahlfors regularity, however. We define the co-dimension 1 Hausdorff measure of a set $E \subset X$ by

$$
\mathcal{H}(E):=\sup _{\delta>0} \mathcal{H}_{\delta}(E)
$$

where for $\delta>0$,

$$
\mathcal{H}_{\delta}(E):=\inf \left\{\sum_{i \in I} \frac{\mu\left(B_{i}\right)}{r_{i}}: B_{i}=B\left(x_{i}, r_{i}\right), r_{i} \leq \delta, E \subset \bigcup_{i \in I} B_{i}\right\} .
$$

The following density results can be proved similarly as in [6, Theorem 2.4.3].

Lemma 2.1. Let $\nu$ be a Radon measure on $X$, let $A \subset X$, and let $t>0$. Then the following hold:

$$
\text { if } \limsup _{r \rightarrow 0} \frac{\nu(B(x, r))}{\mu(B(x, r))} \geq t \quad \text { for all } x \in A \text {, then } \quad \nu(A) \geq t \mathcal{H}(A)
$$

and

$$
\text { if } \limsup _{r \rightarrow 0} r \frac{\nu(B(x, r))}{\mu(B(x, r))} \leq t \quad \text { for all } x \in A, \text { then } \quad \nu(A) \leq C_{d} t \mathcal{H}(A) .
$$

Let $E \subset X$ be a set of finite perimeter. We know that for any Borel set $A \subset X$,

$$
P(E, A)=\int_{\partial^{*} E \cap A} \theta_{E} d \mathcal{H},
$$

where $\theta_{E}: X \rightarrow\left[\alpha, C_{d}\right]$ with $\alpha=\alpha\left(C_{d}, C_{P}\right)>0$, see [1, Theorem 5.3] and [5, Theorem 4.6]. Furthermore, let

$$
\Sigma_{\gamma} E:=\left\{x \in X: \liminf _{r \rightarrow 0} \min \left\{\frac{\mu(B(x, r) \cap E)}{\mu(B(x, r))}, \frac{\mu(B(x, r) \backslash E)}{\mu(B(x, r))}\right\} \geq \gamma\right\}
$$

for a constant $\gamma \in(0,1 / 2]$ depending only on $C_{d}, C_{P}$. Note that $\Sigma_{\gamma} E \subset \partial^{*} E$; by [1, Theorem 5.4] we know that conversely,

$$
\mathcal{H}\left(\partial^{*} E \backslash \Sigma_{\gamma} E\right)=0 .
$$

Lemma 2.2. Let $E \subset X$ be a set of finite perimeter. Then for $\mathcal{H}$-a.e. $x \in \partial^{*} E$ (and thus $P(E, \cdot)$-a.e. $\left.x \in \partial^{*} E\right)$,

$$
\frac{\gamma}{2 C_{P}} \leq \liminf _{r \rightarrow 0} \frac{P(E, B(x, r))}{\mu(B(x, r)) / r} \leq \limsup _{r \rightarrow 0} \frac{P(E, B(x, r))}{\mu(B(x, r)) / r} \leq C_{d}
$$


Proof. The first inequality holds for every $x \in \Sigma_{\gamma} E$ by the relative isoperimetric inequality (2.6). To show the second inequality, note that if $A \subset \partial^{*} E$ and $\varepsilon>0$ are such that

$$
\limsup _{r \rightarrow 0} r \frac{P(E, B(x, r))}{\mu(B(x, r))} \geq C_{d}+\varepsilon
$$

for all $x \in A$, then by the first part of Lemma 2.1 and by $(2.10)$, we have $P(E, A) \geq\left(C_{d}+\varepsilon\right) \mathcal{H}(A)$. However, according to (2.10), we have $P(E, A) \leq C_{d} \mathcal{H}(A)$. Thus we must have $\mathcal{H}(A)=0$.

The lower and upper approximate limits of a function $u$ on $X$ are defined respectively by

$$
u^{\wedge}(x):=\sup \left\{t \in \mathbb{R}: \lim _{r \rightarrow 0} \frac{\mu(B(x, r) \cap\{u<t\})}{\mu(B(x, r))}=0\right\}
$$

and

$$
u^{\vee}(x):=\inf \left\{t \in \mathbb{R}: \lim _{r \rightarrow 0} \frac{\mu(B(x, r) \cap\{u>t\})}{\mu(B(x, r))}=0\right\} .
$$

The jump set $S_{u}$ is defined to be the set where $u^{\wedge}<u^{\vee}$.

By [5, Theorem 5.3], the variation measure of a $B V$ function can be decomposed into the absolutely continuous and singular part, and the latter into the Cantor part and jump part, as follows. Given $u \in B V(X)$, we have for any Borel set $A \subset X$

$$
\begin{aligned}
V(u, A) & =V_{a}(u, A)+V_{s}(u, A) \\
& =V_{a}(u, A)+V_{c}(u, A)+V_{j}(u, A) \\
& =\int_{A} g d \mu+V_{c}(u, A)+\int_{A \cap S_{u}} \int_{u^{\wedge}(x)}^{u^{\vee}(x)} \theta_{\{u>t\}}(x) d t d \mathcal{H}(x),
\end{aligned}
$$

where $g \in L^{1}(X)$ is the density of the absolutely continuous part and the functions $\theta_{\{u>t\}}$ are as in $(2.10)$.

We denote by $B V_{c}(X)$ the class of BV functions with compact support in $X$.

Definition 2.3. We say that $u \in B V(X)$ is a function of least gradient if for all $\varphi \in B V_{c}(X)$,

$$
V(u, \operatorname{supp} \varphi) \leq V(u+\varphi, \operatorname{supp} \varphi) .
$$

Remark 2.4. We end this section by gathering together all the assumptions regarding the metric measure space $(X, d, \mu)$ assumed throughout this paper. We assume that $X$ is a complete, geodesic metric space and that $\mu$ is a doubling measure on $X$, supporting a 1-Poincaré inequality. One of the consequences of assuming that $X$ is complete and $\mu$ is doubling, is that $X$ is then proper, that is, closed and bounded subsets of $X$ are compact. See Remark 6.15 at the end of this paper, regarding the relaxation of some of these assumptions.

\section{Pointed measured Gromov-Hausdorff limits}

In this section we consider tangent spaces of a metric space at a given point. For this, we first need to specify what is meant by the convergence of metric spaces. Existing literature has some slightly different definitions and diverging terminology; here we describe them and provide brief explanation on how these are equivalent. All metric spaces considered here are assumed to be proper. 
Definition 3.1. We say that the sequence of pointed metric spaces $\left(Y_{n}, d_{n}, y_{n}\right)$ converges in the pointed Gromov-Hausdorff distance to the space $\left(Y_{\infty}, d_{\infty}, y_{\infty}\right)$ if for each positive integer $n$ there is a map $\phi_{n}: Y_{\infty} \rightarrow Y_{n}$ so that $\phi_{n}\left(y_{\infty}\right)=y_{n}$, and for each $R>0$ and $\epsilon>0$ there is a positive integer $N_{\epsilon, R}$ such that whenever $k \geq N_{\epsilon, R}$, we have

(1) $\sup _{x, y \in B_{Y_{\infty}}\left(y_{\infty}, R\right)}\left|d_{Y_{k}}\left(\phi_{k}(x), \phi_{k}(y)\right)-d_{Y_{\infty}}(x, y)\right|<\epsilon$

(2) $B_{Y_{k}}\left(y_{k}, R-\epsilon\right) \subset \bigcup_{y \in \phi_{k}\left(B_{Y_{\infty}}\left(y_{\infty}, R\right)\right)} B_{Y_{k}}(y, \epsilon)$.

Note that these maps are not required to be continuous, or even measurable. It is possible to modify $\phi_{n}$ to be measurable, but this is technical, and not necessary for our presentation below.

Remark 3.2. The above definition is compatible with those of [10, 24]. In [10, Definition 8.1.1] and [21, Chapter 11] the following definition of pointed Gromov-Hausdorff convergence was considered: For all $r>0$ and all $0<\epsilon<r$ there exists an $n_{0}=n_{0}(r, \epsilon)$ such that for all $n \geq n_{0}$ there exist functions $\phi_{n}^{\epsilon}: B_{Y_{\infty}}\left(y_{\infty}, r\right) \rightarrow Y_{n}$ with

(1) $\phi_{n}^{\epsilon}\left(y_{\infty}\right)=y_{n}$,

(2) $\left|d_{n}\left(\phi_{n}^{\epsilon}(x), \phi_{n}^{\epsilon}(y)\right)-d_{\infty}(x, y)\right|<\epsilon$ for all $x, y \in B_{Y_{\infty}}\left(y_{\infty}, r\right)$

(3) $B_{Y_{n}}\left(y_{n}, r-\epsilon\right) \subset \bigcup_{y \in \phi_{n}^{\epsilon}\left(B_{Y_{\infty}}\left(y_{\infty}, r\right)\right)} B_{Y_{n}}(y, \epsilon)$.

See [22] for more on pointed Gromov-Hausdorff convergence. To see the compatibility between these two definitions we note that the scales $R$ and $\epsilon$ play the role of localizing the convergence of the tangent spaces. Thus, the second notion is implied by the first, as seen by the choice $\phi_{n}^{\epsilon}:=\phi_{n}$. Conversely, given $\phi_{n}^{\epsilon}$, choosing a sequence of $R_{n}$ monotonically increasing to $\infty$ and $\epsilon_{n}$ monotonically decreasing to 0 , we can even choose $\phi_{n}^{\epsilon}$ to be independent of $\epsilon$ and $r$; hence the equivalence of the notion of [10] with ours. However, in proofs it is often easier to work with the localized versions $\phi_{n}^{\epsilon}$, since it avoids this additional diagonal argument. Where we wish to use globally defined functions, we use $\phi_{n}$. These are interchangeable.

The notion considered in [24] is also equivalent to the above. Since this notion of [24, Definition 2 and Definition 7] is also useful in this paper, especially in defining notions of weak convergence of measures to tangent spaces, we now provide that definition as well. According to [24], the sequence $\left(Y_{n}, d_{n}, y_{n}\right)$ converges to a proper space $\left(Y_{\infty}, d_{\infty}, y_{\infty}\right)$ if there is a proper metric space $\left(Z, d_{Z}\right)$ and a point $z_{0} \in Z$, an isometric embedding $\iota: Y_{\infty} \rightarrow Z$, and for each $n \in \mathbb{N}$ there is an isometric embedding $\iota_{n}: Y_{n} \rightarrow Z$, such that $\iota\left(y_{\infty}\right)=z_{0}=\iota_{n}\left(y_{n}\right)$ and for each $R>0$,

(1) $\lim _{n \rightarrow \infty} \sup _{y \in B_{Y_{n}}\left(y_{n}, R\right)} \operatorname{dist}_{Z}\left(\iota_{n}(y), \iota\left(Y_{\infty}\right)\right)=0$,

(2) $\lim _{n \rightarrow \infty} \sup _{z \in B_{Y_{\infty}}\left(y_{\infty}, R\right)} \operatorname{dist}_{Z}\left(\iota(z), \iota\left(Y_{n}\right)\right)=0$.

From this definition we see that whenever $R, \varepsilon>0$ there is some positive integer $N_{\varepsilon, R}$ such that whenever $n>N_{\varepsilon, R}$, for each $x, y \in B_{Y_{n}}\left(y_{n}, R\right)$ we can find $\widehat{x}, \widehat{y} \in B_{Y_{\infty}}\left(y_{\infty}, R+\varepsilon\right)$ such that

$$
\max \left\{d_{Z}\left(\iota_{n}(x), \iota(\widehat{x})\right), d_{Z}\left(\iota_{n}(y), \iota(\widehat{y})\right)\right\}<\varepsilon, \quad\left|d_{Y_{n}}(x, y)-d_{Y_{\infty}}(\widehat{x}, \widehat{y})\right|<3 \varepsilon .
$$

We also have that for $R>0$ and $\varepsilon>0$ there is some positive integer $N_{\varepsilon, R}$ such that for $n>N_{\varepsilon, R}$, whenever $x, y \in B_{Y_{\infty}}\left(y_{\infty}, R\right)$ there exist $x_{n}, y_{n} \in B_{Y_{n}}\left(y_{n}, R+\varepsilon\right)$ such that

$$
\max \left\{d_{Z}\left(\iota_{n}\left(x_{n}\right), \iota(x)\right), d_{Z}\left(\iota_{n}\left(y_{n}\right), \iota(y)\right)\right\}<\varepsilon, \quad\left|d_{Y_{n}}\left(x_{n}, y_{n}\right)-d_{Y_{\infty}}(x, y)\right|<3 \varepsilon .
$$

This shows that the definition of [24] implies our definition above. The fact that our definition implies the one of [24] comes from the construction of the ambient space $Z$ found in [22], where the 
space $Z$ should be considered to be the completion of the "disjoint union" space $Y$ found in $[22$, Section 4.1.1].

Indeed, we can construct the maps $\iota_{n}$ and $\iota$ from the maps $\phi_{n}$ and vice versa so that the following compatibility condition between these two classes of maps is satisfied: For all $r>0$,

$$
\lim _{n \rightarrow \infty} \sup _{y \in B_{Y_{\infty}}\left(y_{\infty}, r\right)} d_{Z}\left(\iota_{n} \circ \phi_{n}(y), \iota(y)\right)=0 .
$$

For simplicity, and avoiding modifying the space $Z$, as well as the approximating maps $\phi_{n}$, we will generally fix them throughout the exposition below. In order to define other notions, such as convergence of points, curves and functions, passing to a subsequence in $n$ may be necessary. However, this subsequence will be of $n$ and will not require coming up with new $\phi_{n}$ or embedding space $Z$.

In the light of the above discussion, we can say that a sequence, $z_{n} \in Y_{n}$, converges to $z \in Y_{\infty}$ if $\lim _{n \rightarrow \infty} d_{Z}\left(\iota_{n}\left(z_{n}\right), \iota(z)\right)=0$, and see that every $z \in Y_{\infty}$ is a limit of a sequence $z_{n} \in Y_{n}$ as here. By a not-terrible abuse of notation we denote this by

$$
\lim _{n \rightarrow \infty} z_{n}=z .
$$

Next, we define pointed measured Gromov-Hausdorff convergence. For this, we use the embeddings described in the above remark. First consider a sequence of Borel measures $\nu_{n}$ on a metric space $Z$. The measures $\nu_{n}$ converge weakly* to a Borel measure $\nu$ on $Z$ if

$$
\int_{Z} \phi d \nu_{n} \rightarrow \int_{Z} \phi d \nu
$$

as $n \rightarrow \infty$ for all boundedly supported continuous functions $\phi$ on $Z$. We denote this convergence by $\nu_{n} \stackrel{*}{\rightarrow} \nu$.

To define measured Gromov-Hausdorff convergence, we consider the push-forward measures

$$
\iota_{n, *} \nu_{n}(A):=\nu_{n}\left(\iota_{n}^{-1}(A)\right) \text {. }
$$

We say that the sequence of Radon measures $\nu_{n}$ on $Y_{n}$ converges to a Radon measure $\nu_{\infty}$ on $Y_{\infty}$, denoted $\nu_{n} \stackrel{*}{\rightarrow} \nu_{\infty}$, if $\iota_{n, *} \nu_{n} \stackrel{*}{\rightarrow} \iota_{*} \nu_{\infty}$ on $Z$.

Definition 3.3. We say that a sequence of pointed metric measure spaces $\left(Y_{n}, d_{n}, y_{n}, \nu_{n}\right)$ converges pointed measured Gromov-Hausdorff to a space $\left(Y_{\infty}, d_{\infty}, y_{\infty}, \nu_{\infty}\right)$, if the sequence converges in the pointed Gromov-Hausdorff sense, and

$$
\nu_{n} \stackrel{*}{\rightarrow} \nu_{\infty}
$$

Since $Z$ is a proper metric space, it follows that whenever $\sup _{n} \nu_{n}\left(\iota_{n}^{-1}(Z)\right)<\infty$, there is a subsequence $\nu_{n_{k}}$ and a Radon measure $\widehat{\nu_{\infty}}$ on $Z$ such that $\iota_{n_{k}, *} \nu_{n_{k}} \stackrel{*}{\rightarrow} \widehat{\nu_{\infty}}$ in $Z$. This limit measure must have support in $\iota\left(Y_{\infty}\right)$, since the support of $\nu_{\infty}$ is contained in the limit of the supports of $\nu_{n_{k}}$. Indeed, given $\varepsilon>0$ and a radius $R>0$, we know that for large $n$ the set $\iota_{n}\left(B_{Y_{n}}\left(y_{n}, R\right)\right)$ is in an $3 \varepsilon$-neighborhood of $\iota\left(Y_{\infty}\right)$. Recall that $Y_{\infty}$ is a proper metric space. We call such measures $\nu_{\infty}$ limit measures of the sequence $\nu_{n_{k}}$, and they may depend on the choice of the subsequence; the full sequence $\nu_{n}$ may not converge to $\nu_{\infty}$. In the proofs below, we will always pass to the subsequence where this limit holds. The discussion in this paragraph proves the following lemma.

Lemma 3.4. In the situation described in the above paragraph, $\widehat{\nu_{\infty}}\left(Z \backslash \iota\left(Y_{\infty}\right)\right)=0$, and hence there is a Radon measure $\nu_{\infty}$ on $Y_{\infty}$ such that $\widehat{\nu_{\infty}}=\iota_{*} \nu_{\infty}$. 
Definition 3.5. Let $x \in X$ and let $r_{n}>0$ with $r_{n} \rightarrow 0$. Define the sequence of scaled metrics $d_{n}$ on $X$ by

and the scaled measures

$$
d_{n}(y, z):=\frac{d(y, z)}{r_{n}}
$$

$$
\mu_{n}:=\frac{1}{\mu\left(B\left(x, r_{n}\right)\right)} \mu .
$$

If the sequence $\left(X_{n}, d_{n}, x, \mu_{n}\right):=\left(X, d_{n}, x, \mu_{n}\right)$ converges to $\left(X_{\infty}, d_{\infty}, x_{\infty}, \mu_{\infty}\right)$ in the pointed measured Gromov-Hausdorff sense, then we say that $X_{\infty}$ is a tangent space to $X$ at $x$, with tangent measure $\mu_{\infty}$.

We know that if $\mu$ is doubling and $X$ is a complete geodesic space, then by passing to a subsequence of $\left(X, d_{n}, x, \mu_{n}\right)$ if necessary, we will always have a tangent metric measure space as above, which is also geodesic, see [16, Section 6] or the discussion in [21, Section 11]. Note that points of distance less than $r_{n}$ from $x$ in $(X, d)$ are, in the space $X_{n}$, at distance less than 1 from $x$, and that the ball $B\left(x, r_{n}\right)$ has $\mu_{n}$-measure 1 . The tangent space may be non-unique, and depends on the subsequence chosen.

From the work of [24] we know that if $\mu$ is doubling and supports a 1-Poincaré inequality, then for every $x \in X$, all the corresponding tangent spaces have the tangent measure be doubling and support a 1-Poincaré inequality, with the doubling and Poincaré constants depending quantitatively only on the corresponding constants for $X$, see also [21]. A proof of this first appeared in the work [24] of Keith, but he reports in [24] that it was independently found by himself, Koskela, and Cheeger.

We will fix the following notion of a limit of functions.

Definition 3.6. We say that a function $u_{\infty}$ on $X_{\infty}$ is a limit of $u_{n}$ (with $u_{n}$ a function on $X_{n}$ ) if there exists some subsequence $n_{k}$ and $\epsilon_{k} \searrow 0$ such that for all $r>0$

$$
\lim _{k \rightarrow \infty}\left\|u_{\infty}-u_{n_{k}} \circ \phi_{n_{k}}^{\epsilon_{k}}\right\|_{L^{\infty}\left(B_{X_{\infty}}\left(x_{\infty}, r\right)\right)}=0 .
$$

This is equivalent to the following definition of limits using globally defined maps $\phi_{k}$ :

$$
\lim _{k \rightarrow \infty}\left\|u_{\infty}-u_{n_{k}} \circ \phi_{k}\right\|_{L^{\infty}\left(B_{X_{\infty}}\left(x_{\infty}, r\right)\right)}=0 .
$$

Given a sequence of scaled metric measure spaces $\left(X_{n}, d_{n}, x_{n}, \mu_{n}\right)$ pointed Gromov-Hausdorff converging to $\left(X_{\infty}, d_{\infty}, x_{\infty}, \mu_{\infty}\right)$, and $L>0$ such that $u_{n}$ is an $L$-Lipschitz function on $\left(X, d_{n}\right)$, and if $M \geq 0$ such that for each $n \in \mathbb{N}$ we have $\left|u_{n}\left(x_{n}\right)\right| \leq M$, then we can obtain a subsequence of functions $u_{n_{k}}$ on $\left(X_{n_{k}}, d_{n_{k}}\right)$ that converges to an $L$-Lipschitz function on $X_{\infty}$. This is easier to see from the point of view of the definition of [24] as well. For each $n$ we can find a Lipschitz extension $\widehat{u_{n}}$ of $u_{n} \circ \iota_{n}^{-1}$ from $\iota_{n}\left(X_{n}\right)$ to $Z$. We refer the interested reader for a detailed treatment of such an extension in [21, Proposition 11.6.2]. This sequence forms an equibounded and equicontinuous sequence of functions in $Z$ which, being a proper space, lends itself to an application of the Arzelà-Ascoli theorem. Thus we may find a subsequence of $\widehat{u_{n}}$ that converges locally uniformly to a Lipschitz function $\widehat{u_{\infty}}$ on $Z$. We can now choose $u_{\infty}=\widehat{u_{\infty}} \circ \iota$. That this choice of $u_{\infty}$ is a limit of $u_{n}$ follows from the compatibility condition (3.1). These are the heuristics behind the proof of [11, Theorem 10.2], a result which is used in the proof of Theorem 4.8 below.

The notion of limit of functions as given above is concordant with the notion of limit of measures. If $u_{n}$ and $u_{\infty}$ are uniformly Lipschitz and $u_{\infty}$ is a limit of $u_{n_{k}}$, then along the same subsequence $d \nu_{n_{k}}:=u_{n_{k}} d \mu_{n_{k}} \stackrel{*}{\rightarrow} d \nu_{\infty}:=u_{\infty} d \mu_{\infty}$. To see this, note that whenever $\varphi$ is a compactly supported 
Lipschitz function on $Z$ with support $B_{Z}\left(x_{\infty}, r\right)$, where the spaces $X_{n}$ and $X_{\infty}$ isometrically embed as in [22], if $u_{n_{k}} \rightarrow u_{\infty}$ in our sense, then as $\mu_{n_{k}} \stackrel{*}{\rightarrow} \mu_{\infty}$,

$$
\begin{aligned}
\left|\int_{Z} \varphi u_{n_{k}} d \mu_{n_{k}}-\int_{Z} \varphi u_{\infty} d \mu_{\infty}\right| \leq \int_{Z} \| \widehat{u_{n_{k}}} & -u_{\infty} \|_{L^{\infty}\left(B_{Z}\left(x_{\infty}, r\right)\right)}|\varphi| d \mu_{n_{k}} \\
& +\left|\int_{Z} u_{\infty} \varphi d \mu_{n_{k}}-\int_{Z} u_{\infty} \varphi d \mu_{\infty}\right| \rightarrow 0 \text { as } k \rightarrow \infty .
\end{aligned}
$$

As before, these functions depend on the subsequence chosen. In fact, there are several dependencies. The very tangent spaces $X_{\infty}$ and the tangent measures $\mu_{\infty}$ depend on the subsequence of blow ups $r_{n} \searrow 0$. Indeed, different sequences can lead to different tangent spaces that are not isometric to each other, see for instance the discussion surrounding [31, Theorem 1.4]. Once this sequence $\left(r_{n}\right)_{n}$ is chosen, every choice of maps $\iota_{n}$ and $\phi_{n}^{\epsilon_{k}}$ leads to the same isometry classes of the tangent space $X_{\infty}$ and the tangent measure $\mu_{\infty}$. However, especially when blowing up functions $u_{n}$ and measures $u_{n} d \mu_{n}$, one should be careful about this and choose a further subsequence and the maps, as a sequence of functions may converge with one choice of maps $\iota_{n}, \phi_{n}^{\epsilon_{k}}$ and not converge with another choice. We will always assume that all these choices are given to us.

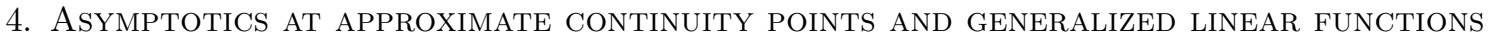

The goal of this section is to study the asymptotic behavior of BV functions at points outside the jump and Cantor parts of their variation measures. We start with the following handy lemma.

Lemma 4.1. Let $u, v \in B V(X)$. Suppose $E \subset X$ is a Borel set such that for each $x \in E$ we have

$$
\lim _{r \rightarrow 0^{+}} \frac{\mu(B(x, r) \cap E)}{\mu(B(x, r))}=1,
$$

and $u(x)=v(x)$. Then $V(u-v, E)=0$ and so for each $A \subset E$ we have $V(u, A)=V(v, A)$.

Proof. From the above, we know that for each $t \in \mathbb{R}$ the set $E \cap \partial^{*} E_{t}$ is empty, where $E_{t}=\{x \in$ $X: u(x)-v(x)>t\}$. Therefore by the coarea formula (2.5) and by (2.10), the claim follows.

In the Euclidean setting we know that for $\mathcal{L}^{n}$-a.e. $x \in \mathbb{R}^{n}$ (where $\mathcal{L}^{n}$ denotes the $n$-dimensional Lebesgue measure) a BV function converges under blow-up to a linear function (see e.g. [3, Theorem 3.83]). In the metric setting, for $p>1$ the notion of linear function is interpreted as a function that is constant or else satisfies the following two properties: (a) the image of $X$ under the function is $\mathbb{R}$, and (b) the minimal $p$-weak upper gradient of the function is constant (and given that we have a Poincaré inequality, this constant should be non-zero if the function is not the constant function); see for example [11, Section 10]. It was shown in [11, Theorem 10.2] that given a Lipschitz function, any asymptotic limit of that function at almost every point yields such a linear function on the corresponding tangent space, which we defined in Section 3. In the case $p=1$, which is the natural setting for BV functions, we will prove that the asymptotic limits are the so-called generalized linear functions on the tangent spaces. by

For $g \in L_{\text {loc }}^{1}(X)$ nonnegative and $R>0$, we define the restricted maximal function of $g$ at $x \in X$

$$
\mathcal{M}_{R} g(x):=\sup _{0<r \leq R} f_{B(x, r)} g d \mu
$$


The maximal function of a Radon measure $\nu$ is defined similarly by

$$
\mathcal{M}_{R} \nu(x):=\sup _{0<r \leq R} \frac{\nu(B(x, r))}{\mu(B(x, r))} .
$$

Recall that if $u \in B V(X)$, then

$$
d V(u, \cdot)=g d \mu+d V_{s}(u, \cdot),
$$

where $g \in L^{1}(X)$ is the Radon-Nikodym derivative of $V(u, \cdot)$ with respect to $\mu$ and $V_{s}(u, \cdot)$ is the singular part.

Proposition 4.2. Let $u \in B V(X)$. Then for $\mu$-a.e. $x \in X$ for which $g(x)>0$ there exists $R>0$ and a set $A_{x} \not \supset x$ with density 0 at the point $x$ such that $\left.u\right|_{B(x, R) \backslash A_{x}}$ is Lipschitz with constant $C g(x)$. For $\mu$-a.e. $x \in X$ for which $g(x)=0$, for each $\delta>0$ there is a set $A_{x, \delta} \not \supset x$ with density 0 at $x$ such that $\left.u\right|_{B(x, R) \backslash A_{x, \delta}}$ is Lipschitz with constant $\delta$.

Proof. We follow the proof of [21, Proposition 13.5.2]. For $\mu$-a.e. $x \in X$, we have

$$
\lim _{r \rightarrow 0} f_{B(x, r)}|g-g(x)| d \mu=0 \quad \text { and } \quad \lim _{r \rightarrow 0} \frac{V_{s}(u, B(x, r))}{\mu(B(x, r))}=0
$$

by the Lebesgue-Radon-Nikodym theorem, see e.g. [21, Section 3.4]. Fix such $x \in X$ for which also $g(x)>0$.

Let $R>0$ and let $y, z \in B(x, R)$ be Lebesgue points of $u$. For nonnegative integers $i$ we set $B_{i}:=B\left(y, 2^{-i} d(y, z)\right)$, and for negative integers $i$ we set $B_{i}:=B\left(z, 2^{i} d(y, z)\right)$. Then, by the doubling property of $\mu$ and the Poincaré inequality (2.7),

$$
\begin{aligned}
|u(y)-u(z)| & \leq \sum_{i \in \mathbb{Z}}\left|u_{B_{i}}-u_{B_{i+1}}\right| \\
& \leq C \sum_{i \in \mathbb{Z}} f_{2 B_{i}}\left|u-u_{2 B_{i}}\right| d \mu \\
& \leq C d(y, z) \sum_{i \in \mathbb{Z}} 2^{-|i|} \frac{V\left(u, 2 B_{i}\right)}{\mu\left(2 B_{i}\right)} \\
& =C d(y, z) \sum_{i \in \mathbb{Z}} 2^{-|i|}\left(f_{2 B_{i}} g d \mu+\frac{V_{s}\left(u, 2 B_{i}\right)}{\mu\left(2 B_{i}\right)}\right) \\
& \leq C d(y, z) \sum_{i \in \mathbb{Z}} 2^{-|i|}\left(f_{2 B_{i}}|g-g(x)| d \mu+\frac{V_{s}\left(u, 2 B_{i}\right)}{\mu\left(2 B_{i}\right)}\right)+C d(y, z) g(x) .
\end{aligned}
$$

For $s>0$, set

$$
\tau_{s}:=\sup _{0<r \leq s}\left(f_{B(x, r)}|g-g(x)| d \mu+\frac{V_{s}(u, B(x, r))}{\mu(B(x, r))}\right) .
$$

Note that since $\mu$ is doubling, for any $s>0$ and any Radon measure $\nu$ we have

$$
\mathcal{M}_{s} \nu(y) \leq C \mathcal{M}_{2 d(x, y)} \nu(y)+C \mathcal{M}_{2 s} \nu(x) .
$$


Applying this with $s=2 d(y, z)<4 R$ in the second inequality below, we get

$$
\begin{aligned}
|u(y)-u(z)| \leq & C d(y, z)\left[g(x)+\mathcal{M}_{2 d(y, z)}|g-g(x)|(y)+\mathcal{M}_{2 d(y, z)}|g-g(x)|(z)\right. \\
& \left.+\mathcal{M}_{2 d(y, z)} V_{s}(u, \cdot)(y)+\mathcal{M}_{2 d(y, z)} V_{s}(u, \cdot)(z)\right] \\
\leq & C d(y, z)\left[g(x)+\mathcal{M}_{2 d(x, y)}|g-g(x)|(y)+\mathcal{M}_{2 d(x, z)}|g-g(x)|(z)\right. \\
& \left.+\mathcal{M}_{2 d(x, y)} V_{s}(u, \cdot)(y)+\mathcal{M}_{2 d(x, z)} V_{s}(u, \cdot)(z)+\mathcal{M}_{8 R}[g-g(x)](x)+\mathcal{M}_{8 R} V_{s}(u, \cdot)(x)\right] \\
\leq & C d(y, z)\left[g(x)+\mathcal{M}_{2 d(x, y)}|g-g(x)|(y)+\mathcal{M}_{2 d(x, z)}|g-g(x)|(z)\right. \\
& \left.+\mathcal{M}_{2 d(x, y)} V_{s}(u, \cdot)(y)+\mathcal{M}_{2 d(x, z)} V_{s}(u, \cdot)(z)+\tau_{8 R}\right] .
\end{aligned}
$$

We only consider $R>0$ to be small enough so that $\tau_{8 R}<g(x)$ (here we need the fact that $g(x)>0)$. We choose a sequence of radii $R_{M} \searrow 0$ as $M \rightarrow \infty$ such that $2^{M} \tau_{8 R_{M}}<g(x)$ for each $M \in \mathbb{N}$. Next let $A_{M}$ be the set of all points $y \in B\left(x, R_{M}\right)$ such that for some $0<r \leq 2 d(x, y)$,

$$
f_{B(y, r)}|g-g(x)| d \mu+\frac{V_{s}(u, B(y, r))}{\mu(B(y, r))}>2^{M} \tau_{8 R_{M}} .
$$

For each $y \in A_{M}$ there is a ball $B\left(y, r_{y}\right)$ with $0<r_{y} \leq 2 d(x, y)<2 R_{M}$ such that the above inequality holds, and so the family $\left\{B\left(y, r_{y}\right)\right\}_{y \in A_{M}}$ is a cover of $A_{M}$. By the 5 -covering theorem we can extract a countable, pairwise disjoint subfamily $\mathcal{G}$ of the above family such that $A_{M} \subset \bigcup_{B \in \mathcal{G}} 5 B$. If $\tau_{8 R_{M}}=0$, then $\mu\left(A_{M}\right)=0$; else we see by the doubling property of $\mu$ that

$$
\begin{aligned}
\mu\left(A_{M}\right) & \leq C \sum_{B \in \mathcal{G}} \mu(B) \\
& \leq \frac{C}{2^{M} \tau_{8 R_{M}}} \sum_{B \in \mathcal{G}}\left(\int_{B}|g-g(x)| d \mu+V_{s}(u, B)\right) \\
& \leq \frac{C}{2^{M} \tau_{8 R_{M}}}\left(\int_{B\left(x, 4 R_{M}\right)}|g-g(x)| d \mu+V_{s}\left(u, B\left(x, 4 R_{M}\right)\right)\right) \\
& \leq \frac{C \mu\left(B\left(x, 4 R_{M}\right)\right)}{2^{M} \tau_{8 R_{M}}}\left(f_{B\left(x, 4 R_{M}\right)}|g-g(x)| d \mu+\frac{V_{s}\left(u, B\left(x, 4 R_{M}\right)\right)}{\mu\left(B\left(x, 4 R_{M}\right)\right)}\right) \\
& \leq \frac{C \mu\left(B\left(x, R_{M}\right)\right)}{2^{M} \tau_{8 R_{M}}} \tau_{4 R_{M}} \leq \frac{C \mu\left(B\left(x, R_{M}\right)\right)}{2^{M}} .
\end{aligned}
$$

We can add to each $A_{M}$ all the non-Lebesgue points of $u$ different from $x$, without adding measure. Now note that $x \notin A_{M}$ and that by (4.3), $u$ is $C g(x)$-Lipschitz in $B\left(x, R_{M}\right) \backslash A_{M}$. By choosing

$$
A_{x}:=\bigcup_{M=1}^{\infty} A_{M} \backslash B\left(x, R_{M+1}\right)
$$

we see that $u$ is $C g(x)$-Lipschitz in $B\left(x, R_{1}\right) \backslash A_{x}$. Indeed, if $y, z \in B\left(x, R_{1}\right) \backslash A_{x}$ such that $y \neq x \neq z$, then there are positive integers $M_{1}$ and $M_{2}$ such that $y \in B\left(x, R_{M_{1}}\right) \backslash B\left(x, R_{M_{1}+1}\right)$ with $y \notin A_{M_{1}}$ and $z \in B\left(x, R_{M_{2}}\right) \backslash B\left(x, R_{M_{2}+1}\right)$ with $z \notin A_{M_{2}}$. We can assume that $M_{2}>M_{1}$. It then follows from (4.3) that

$$
|u(y)-u(z)| \leq C d(y, z)\left[g(x)+2^{M_{1}} \tau_{8 R_{M_{1}}}+2^{M_{2}} \tau_{8 R_{M_{2}}}+\tau_{8 R_{M_{1}}}\right] \leq 4 C g(x) d(y, z) .
$$

Therefore $u$ is $C g(x)$-Lipschitz continuous in $B\left(x, R_{1}\right) \backslash\left(A_{x} \cup\{x\}\right)$. The fact that $u$ is approximately continuous at $x$, together with the fact that $A_{x}$ has lower density zero at $x$ (see the argument below), tells us that $u$ is $C g(x)$-Lipschitz continuous in $B\left(x, R_{1}\right) \backslash A_{x}$. 
Moreover,

$$
\frac{\mu\left(A_{x} \cap B\left(x, R_{M_{0}}\right)\right)}{\mu\left(B\left(x, R_{M_{0}}\right)\right)} \leq C \sum_{M=M_{0}}^{\infty} \frac{\mu\left(A_{M}\right)}{\mu\left(B\left(x, R_{M}\right)\right)} \leq C \sum_{M=M_{0}}^{\infty} 2^{-M} \rightarrow 0 \quad \text { as } M_{0} \rightarrow \infty .
$$

This guarantees that $A_{x}$ has lower density 0 at $x$. On the other hand, by the choice of the covering of $A_{M}$ by balls $B\left(y, r_{y}\right)$ with radius $r_{y} \leq 2 d(x, y)$, in the estimate for $\mu\left(A_{M}\right)$ obtained above we can in fact obtain for any $0<r \leq R_{M}$ that

$$
\mu\left(A_{M} \cap B(x, r)\right) \leq \frac{C}{2^{M}} \mu(B(x, 4 r)) \leq \frac{C C_{d}^{2}}{2^{M}} \mu(B(x, r)) .
$$

This guarantees that $A_{x}$ has density 0 at $x$. Choosing $R=R_{1}$, we have proved the first claim.

Finally, if $\{x \in X: g(x)=0\}$ has positive measure, then the above argument gives that for $\mu$-a.e. $x$ in this set, for every $\delta>0$ there exists $A_{x, \delta}$ with $x \notin A_{x, \delta}$ and $A_{x, \delta}$ is of density 0 at $x$ such that $\left.u\right|_{B(x, R) \backslash A_{x, \delta}}$ is $\delta$-Lipschitz.

Lemma 4.3. Let $u \in B V(X)$. For $\mu$-a.e. $x \in X$ the following holds: if $A \subset X$ has density 0 at $x$, then

$$
\lim _{r \rightarrow 0} \frac{1}{r} \frac{1}{\mu(B(x, r))} \int_{A \cap B(x, r)}|u-u(x)| d \mu=0 .
$$

Proof. Excluding a $\mu$-negligible set, we can take a Lebesgue point $x$ of $u$ such that (just as in (4.2))

$$
\lim _{r \rightarrow 0} \frac{V(u, B(x, r))}{\mu(B(x, r))}=g(x) .
$$

By Hölder's inequality,

$$
\frac{1}{r} \frac{1}{\mu(B(x, r))} \int_{A \cap B(x, r)}|u-u(x)| d \mu \leq \frac{1}{r}\left(f_{B(x, r)}|u-u(x)|^{Q /(Q-1)} d \mu\right)^{(Q-1) / Q}\left(\frac{\mu(A \cap B(x, r))}{\mu(B(x, r))}\right)^{1 / Q} .
$$

Since $x$ is a Lebesgue point of $u$, by the Sobolev-Poincaré inequality (2.7),

$$
\begin{aligned}
\left(f_{B(x, r)}|u-u(x)|^{Q /(Q-1)} d \mu\right)^{\frac{Q-1}{Q}} \leq & \left(f_{B(x, r)}\left|u-u_{B(x, r)}\right|^{Q /(Q-1)} d \mu\right)^{\frac{Q-1}{Q}} \\
& +\sum_{j=1}^{\infty}\left(f_{B(x, r)} \mid u_{B\left(x, 2^{-j+1} r\right)}-u_{\left.\left.B\left(x, 2^{-j} r\right)\right|^{Q /(Q-1)} d \mu\right)^{\frac{Q-1}{Q}}}\right. \\
\leq & C r \frac{V(u, B(x, r))}{\mu(B(x, r))}+\sum_{j=1}^{\infty}\left|u_{B\left(x, 2^{-j+1} r\right)}-u_{B\left(x, 2^{-j} r\right)}\right| \\
\leq & C r \frac{V(u, B(x, r))}{\mu(B(x, r))}+C \sum_{j=1}^{\infty} 2^{-j+1} r \frac{V\left(u, B\left(x, 2^{-j+1} r\right)\right)}{\mu\left(B\left(x, 2^{-j+1} r\right)\right)} \\
\leq & C r \mathcal{M}_{r} V(u, \cdot)(x) .
\end{aligned}
$$

Thus we get

$$
\frac{1}{r} \frac{1}{\mu(B(x, r))} \int_{A \cap B(x, r)}|u-u(x)| d \mu \leq C \mathcal{M}_{r} V(u, \cdot)(x)\left(\frac{\mu(A \cap B(x, r))}{\mu(B(x, r))}\right)^{1 / Q} .
$$


Note that by $(4.5), \lim _{r \rightarrow 0} \mathcal{M}_{r} V(u, \cdot)(x)=g(x)<\infty$, and so by the fact that $A$ has density 0 at $x$, we get the conclusion.

Definition 4.4. Let $v$ be a real-valued function on a metric space $(Z, d)$. The oscillation of $v$ in a ball $B(x, r)$ is

$$
\underset{(x, r)}{\operatorname{osc} v}:=\sup _{y \in B(x, r)} \frac{|v(y)-v(x)|}{r} .
$$

We also set

$$
\operatorname{LIP} v:=\sup _{y, z \in Z: y \neq z} \frac{|v(y)-v(z)|}{d(y, z)} .
$$

Observe that $\underset{(x, r)}{\operatorname{osc} v} \leq \operatorname{LIP} v$.

We now return to the sequence $X_{n}$ of zoomed-in versions of $X$, as defined in Section 3. For $u \in B V(X)$, we wish to study the limit of the functions

$$
u_{n}(y):=\frac{u(y)-u(x)}{r_{n}}
$$

that are defined on $X_{n}$. Suppose the point $x$ satisfies the conclusion of Proposition 4.2. Zooming in and defining $u_{n}$ as above, we note that $u$ is only known to be Lipschitz continuous on $B(x, R) \backslash A_{x}$. This poses a problem for studying the supposed limit function $u_{\infty}$. Though the set $A_{x}$ from Proposition 4.2 has zero density at point $x$, it could still be very much in the image of the functions $\phi_{n}^{\epsilon}$ used for comparing $u_{n}$ with $u_{\infty}$ (note that the $\phi_{n}^{\epsilon}$ are not necessarily continuous). This could have the effect of a limit function $u_{\infty}$ having little to do with the values of $u$ outside $A_{x}$, a set which is quantitatively marginal as we have shown. It seems prudent to search for a limit function $u_{\infty}$ that reflects the values of $u$ on $B(x, R) \backslash A_{x}$ if we want to explore any properties of this limit function. With that in mind, we make the following definition:

Definition 4.5. For $x \in X$ for which $g(x)>0$ and the conclusion of Proposition 4.2 holds, we say that the functions $\phi_{n}$ in the definition of pointed measured Gromov-Hausdorff convergence are adapted to $u$ if for each $R>0$ and $\epsilon>0$ there is a positive integer $N_{\epsilon, R}$ such that

$$
\phi_{n}^{\epsilon}\left(B_{X_{\infty}}\left(x_{\infty}, R\right)\right) \cap A_{x}=\emptyset
$$

for all $n \geq N_{\epsilon, R}$.

Thanks to the following lemma we know that whenever $\left(X_{\infty}, d_{\infty}, x_{\infty}, \mu_{\infty}\right)$ is a tangent space to $X$ at $x$ as in Definition 3.5, we can always find a subsequence of the sequence $\left(X_{n}, d_{n}, x, \mu_{n}\right)$ such that the corresponding maps $\phi_{n}$ are adapted to $u$.

Lemma 4.6. Suppose $u \in B V(X)$ and $x \in X$ is a point for which $g(x)>0$ and the conclusion of Proposition 4.2 holds. If $\left(X_{\infty}, d_{\infty}, x_{\infty}, \mu_{\infty}\right)$ is a pointed measured Gromov-Hausdorff limit of $\left(X, d_{n}, x, \mu_{n}\right)$ for some positive sequence $r_{n} \rightarrow 0$, then there exist functions $\phi_{n}$ that are adapted to $u$ at $x$.

Proof. Assume for simplicity that $R=1 / 2$ and fix $0<\epsilon \leq 1$. By the doubling condition, we have that

$$
\frac{\mu\left(B(x, 2 s) \cap A_{x}\right)}{\mu(B(x, s))} \rightarrow 0 \quad \text { as } s \rightarrow 0 .
$$


By (2.2), there exists $Q>1$ such that whenever $y \in B(x, s)$ and $0<t \leq s$,

$$
\frac{1}{C}\left(\frac{t}{s}\right)^{Q} \leq \frac{\mu(B(y, t))}{\mu(B(x, s))}
$$

It follows that if $y \in B\left(x, r_{n}\right)$ (i.e. $\left.y \in B_{n}(x, 1)\right)$, then

$$
\frac{1}{C} \epsilon^{Q} \leq \frac{\mu\left(B\left(y, \epsilon r_{n}\right)\right)}{\mu\left(B\left(x, r_{n}\right)\right)}=\mu_{n}\left(B_{n}(y, \epsilon)\right),
$$

where $B_{n}$ is the ball in the metric $d_{n}=r_{n}^{-1} d$. On the other hand, (4.6) implies that for sufficiently small $r_{n}$,

$$
\mu_{n}\left(B_{n}(x, 2) \cap A_{x}\right)=\frac{\mu\left(B\left(x, 2 r_{n}\right) \cap A_{x}\right)}{\mu\left(B\left(x, r_{n}\right)\right)}<\frac{1}{C} \epsilon^{Q} .
$$

It follows that for such $n$, the set $B_{n}(y, \epsilon) \backslash A_{x}$ has positive measure and therefore cannot be empty. That is, $X \backslash A_{x}$ is $\epsilon$-dense in $B_{n}(x, 1)$.

The points in $A_{x}$ can be easily avoided by redefining the approximating isometries $\phi_{n}$ such that points (1) and (2) of Definition 3.1 still hold, but with $3 \epsilon$ rather than $\epsilon$.

Lemma 4.7. Let $v$ be a Lipschitz function on a metric measure space $\left(Z, d_{Z}, \mu_{Z}\right)$, where $\mu_{Z}$ is doubling. Suppose that $K \subset Z$ and $z \in Z$ such that

$$
\lim _{r \rightarrow 0^{+}} \frac{\mu_{Z}(B(z, r) \cap K)}{\mu_{Z}(B(z, r))}=0 .
$$

Then

$$
\operatorname{Lip} v(z):=\limsup _{Z \ni y \rightarrow z} \frac{|v(z)-v(y)|}{d_{Z}(y, z)}=\limsup _{Z \backslash K \ni y \rightarrow z} \frac{|v(z)-v(y)|}{d_{Z}(y, z)} .
$$

Proof. Clearly

$$
\limsup _{Z \ni y \rightarrow z} \frac{|v(z)-v(y)|}{d_{Z}(y, z)} \geq \limsup _{Z \backslash K \ni y \rightarrow z} \frac{|v(z)-v(y)|}{d_{Z}(y, z)} .
$$

Let $y_{i} \in Z$ be a sequence converging to $z$ such that

$$
\frac{\left|v(z)-v\left(y_{i}\right)\right|}{d_{Z}\left(y_{i}, z\right)} \rightarrow \limsup _{Z \ni y \rightarrow z} \frac{|v(z)-v(y)|}{d_{Z}(y, z)} .
$$

If we have a subsequence of this sequence that lies in $Z \backslash K$, then we have the desired equality. So suppose without loss of generality that each $y_{i} \in K$. We claim that for each $\varepsilon>0$ there is some positive integer $N_{\varepsilon}$ such that when $i \geq N_{\varepsilon}$, we have $d_{Z}\left(w, y_{i}\right) \leq \varepsilon d_{Z}\left(z, y_{i}\right)$ for some $w \in Z \backslash K$. Indeed, if this is not the case, then there is a positive number $\varepsilon_{0}$ and a subsequence $i_{k}$ such that $B\left(y_{i_{k}}, \varepsilon_{0} d_{Z}\left(z, y_{i_{k}}\right)\right) \subset K$, in which case by the doubling property of $\mu_{Z}$ we have

$$
\limsup _{r \rightarrow 0^{+}} \frac{\mu_{Z}(B(z, r) \cap K)}{\mu_{Z}(B(z, r))} \geq \frac{1}{C_{d}^{\alpha}}>0
$$

where $\alpha$ is the real number for which $2^{\alpha} \varepsilon_{0} \geq 4$. This would violate the assumption on the density of $K$ at $z$. 
Now fixing $\varepsilon>0$, with $w_{i} \in Z \backslash K$ such that $d_{Z}\left(y_{i}, w_{i}\right) \leq \varepsilon d_{Z}\left(z, y_{i}\right)$, we have

$$
\begin{aligned}
\frac{\left|v(z)-v\left(y_{i}\right)\right|}{d_{Z}\left(y_{i}, z\right)} \leq \frac{\left|v(z)-v\left(w_{i}\right)\right|}{d_{Z}\left(z, w_{i}\right)} \frac{d_{Z}\left(z, w_{i}\right)}{d_{Z}\left(y_{i}, z\right)}+\frac{\left|v\left(w_{i}\right)-v\left(y_{i}\right)\right|}{d_{Z}\left(y_{i}, z\right)} & \leq \frac{\left|v(z)-v\left(w_{i}\right)\right|}{d_{Z}\left(z, w_{i}\right)} \frac{d_{Z}\left(z, w_{i}\right)}{d_{Z}\left(y_{i}, z\right)}+L \varepsilon \\
& \leq \frac{\left|v(z)-v\left(w_{i}\right)\right|}{d_{Z}\left(z, w_{i}\right)}[1+\varepsilon]+L \varepsilon
\end{aligned}
$$

where $L$ is a Lipschitz constant of $v$. Letting $i \rightarrow \infty$ followed by $\varepsilon \rightarrow 0^{+}$gives the desired identity.

Now we wish to speak about functions $u_{\infty}$ that are limits of $u_{n}$ according to (3.3), with the functions $\phi_{n}$ adapted to $u$. Note that the values of $u_{n}$ on $A_{x}$ are not considered in evaluating (3.3) by the maps $\phi_{n}$.

By Hölder's inequality, the 1-Poincaré inequality implies the $p$-Poincaré inequality for all $1<$ $p<\infty$. By [26, Proposition 4.3], for each $k \in \mathbb{N}$ there is a Lipschitz function $v_{k} \in B V(X)$ such that

$$
\mu\left(\left\{y \in X: u(y) \neq v_{k}(y)\right\}\right)<1 / k .
$$

Since for any measurable set $K \subset X$ we have that the upper density of $K$ at almost every point in $X \backslash K$ is zero, by modifying the set $K_{k}=\left\{y \in X: u(y) \neq v_{k}(y)\right\}$ on a set of measure zero we can assume that $\mu\left(K_{k}\right)<1 / k$ and that for every $x \in X \backslash K_{k}$ we have

$$
\limsup _{r \rightarrow 0^{+}} \frac{\mu\left(B(x, r) \cap K_{k}\right)}{\mu(B(x, r))}=0
$$

and that $v_{k}$ is asymptotically generalized linear in the sense of [11, Theorem 10.2] (see also [11, Definition 8.1 and Definition 10.1]) and that the analysis of Proposition 4.2 holds for $x$. By further enlarging $K_{k}$ if necessary (without increasing its measure), we can also assume that $x$ is a Lebesgue point of Lip $v_{k}$. Since both $K_{k}$ and $A_{x}$ have upper density zero at $x$, from Lemma 4.7 we know that $x$ is a Lebesgue point also for Lip $\widetilde{u}$ of any Lipschitz extension $\widetilde{u}$ of $\left.u\right|_{B(x, R) \backslash\left[A_{x} \cup K_{k}\right]}$ to $B(x, R)$. Note that we then have by Lemma 4.7 that

$$
\left.\operatorname{Lip} u\right|_{B(x, R) \backslash\left(A_{x} \cup K_{k}\right)}(x)=\operatorname{Lip} \widetilde{u}(x)=\operatorname{Lip} v_{k}(x) .
$$

Thus the theory developed in [11, Section 10] is applicable for $x \in X \backslash K_{k}$. Let $N=\bigcap_{k \in \mathbb{N}} K_{k}$. Then $\mu(N)=0$, and for each $x \in X \backslash N$ the theory developed in [11, Section 10] is applicable. Therefore by [11, Theorem 10.2], this immediately implies the equality of upper and lower Lipschitz constants for $\widetilde{u}$, and this Lipschitz constant $\operatorname{Lip} u_{\infty}$ is indeed a constant on $X_{\infty}$, and that

$$
\operatorname{Lip} u_{\infty} \equiv \operatorname{Lip} \widetilde{u}(x)=\left.\operatorname{Lip} u\right|_{B(x, R) \backslash A_{x}}(x),
$$

and this constant minimal $p$-weak upper gradient, for any $1<p<\infty$, of $u_{\infty}$ is bounded above by $C g(x)$ thanks to Proposition 4.2. On the other hand, by the lower semicontinuity of BV energy, we know that $d V\left(v_{k}, \cdot\right) \leq \operatorname{Lip} v_{k} d \mu$, and so the Radon-Nikodym derivative of $V\left(v_{k}, \cdot\right)$ with respect to $\mu$, which by Lemma 4.1 is also equal to the Radon-Nikodym derivative $g$ of $V(u, \cdot)$ with respect to $\mu$ in $X \backslash K_{k}$, is bounded above by $\operatorname{Lip} v_{k}$. Therefore we have

$$
g(x) \leq \operatorname{Lip} u_{\infty} \leq C g(x) .
$$

We collect these observations below.

Theorem 4.8. Let $u \in B V(X)$. Then for $\mu$-a.e. $x \in X$ and any tangent space $\left(X_{\infty}, d_{\infty}, x_{\infty}, \mu_{\infty}\right)$, any function $u_{\infty}$ that arises as a limit adapted to $u$ at $x$ has a constant minimal p-weak upper gradient for each $p>1$ and that constant is less than $C g(x)$, where $C$ is as in Proposition 4.2. 
Furthermore, with $h$ the minimal 1-weak upper gradient of $u_{\infty}$, we have that $L /\left(4 C_{0}\right) \leq h \leq L$ where $L$ is the constant minimal p-weak upper gradient, and $C_{0}$ depends solely on the doubling and the 1-Poincaré constants of $X_{\infty}$.

Proof. For $\mu$-a.e. $x \in X$ at which $g(x)>0$, the proof of the first part of the theorem follows from the discussions above. By Proposition 4.2, at $\mu$-a.e. $x \in X$ at which $g(x)=0$ we have that for every $\delta>0$, we can extend $\left(\left.u\right|_{B(x, R) \backslash A_{x, \delta}}\right) \circ \iota_{n}^{-1}$ as a $\delta$-Lipschitz function $U_{n}$ to $Z$, and so any limit $U_{\infty}$ of a subsequence (at least one limit exists thanks to the Arzela-Ascoli theorem) is also $\delta$-Lipschitz on $Z$. It follows that $u_{\infty}=U_{\infty} \circ \iota$ is $\delta$-Lipschitz continuous on $X_{\infty}$. Since this holds for each $\delta>0$, we have that $u_{\infty}$ is 0-Lipschitz continuous, that is, it is constant. It follows then again that its minimal 1-weak upper gradient is the constant function $0=g(x)$.

Thus it now suffices to prove the last statement of the theorem at the points where $g$ is positive. From a telescoping argument for the Lipschitz function $u_{\infty}$ (see for example [21, Proof of Theorem 8.1.7(iii)]) on $X_{\infty}$, we see that whenever $\varepsilon>0$, for $z, w \in X_{\infty}$ with $d(z, w)<\varepsilon$ we have

$$
\left|u_{\infty}(z)-u_{\infty}(w)\right| \leq C_{0} d_{X_{\infty}}(z, w)\left[\mathcal{M}_{4 \varepsilon} h(z)+\mathcal{M}_{4 \varepsilon} h(w)\right]
$$

where, as in (4.1), $\mathcal{M}_{r} h(o):=\sup _{0<\rho \leq r} f_{B(o, \rho)} h d \mu_{\infty}$ for $o \in X_{\infty}$. Thus it follows from the local version of [21, Theorem 10.2.8] that $4 C_{0} \mathcal{M}_{4 \varepsilon} h$ is an upper gradient of $u_{\infty}$. Therefore by the minimality of the constant function $L$ as a $p$-weak upper gradient of $u_{\infty}$, we see that $L \leq 4 C_{0} \mathcal{M}_{4 \varepsilon} h$ for each $\varepsilon>0$. Letting $\varepsilon \rightarrow 0$ and invoking the Lebesgue differentiation theorem, we see that $L \leq 4 C_{0} h$. Finally, as $u_{\infty}$ is Lipschitz, the constant function $L$ is also equal to Lip $u_{\infty}$ which is also an upper gradient of $u_{\infty}$, and so by the minimality of $h$ as a 1 -weak upper gradient, we see that $h \leq L$, completing the proof.

Theorem 4.9. Let $u \in B V(X)$. Then for $\mu$-a.e. $x \in X$ for which $g(x)>0$, and for every tangent space $\left(X_{\infty}, d_{\infty}, x_{\infty}, \mu_{\infty}\right)$, any function $u_{\infty}$ that arises as a limit adapted to $u$ at $x$ satisfies $u_{\infty}\left(x_{\infty}\right)=0$ and

$$
\frac{g(x)}{C} \leq \underset{B(y, s)}{\operatorname{Osc}} u_{\infty} \leq \operatorname{LIP} u_{\infty} \leq C g(x)
$$

for every $y \in X_{\infty}$ and $s>0$. Furthermore, $u_{\infty}$ is a function of least gradient. For $\mu$-a.e. $x \in X$ for which $g(x)=0, u_{\infty}$ is a constant function.

Proof. The inequality $\underset{B(y, s)}{\operatorname{osc}} u_{\infty} \leq \operatorname{LIP} u_{\infty}$ is true by definition, and the inequality LIP $u_{\infty} \leq C g(x)$ follows from Proposition 4.2 and [11, Theorem 10.2] applied to the blow-up of the Lipschitz function $\widetilde{u}$. For the inequality $\frac{g(x)}{C} \leq \underset{(y, s)}{\operatorname{osc}} u_{\infty}$ we first note that by [23, Theorem 6.2.1],

$$
\operatorname{lip} \widetilde{u}(x) \leq \underset{B(y, s)}{\operatorname{Osc}} u_{\infty},
$$

where $\widetilde{u}$ is a McShane extension of $\left.u\right|_{B(x, 1) \backslash A_{x}}$ to $B(x, 1)$. Now note by Lemma 4.1 that

$$
\frac{g(x)}{C} \leq \operatorname{lip} \widetilde{u}(x)
$$

By [11, Theorem 10.2] we know that $u_{\infty}$ is generalized linear and hence is $p$-harmonic for each $p>1$ (see [11, Definition 8.1]). Letting $p \rightarrow 1^{+}$, it follows from [28, Theorem 3.3] that $u_{\infty}$ is a function of least gradient in $X_{\infty}$.

Finally, if $g(x)=0$ and $x$ is a point of density 1 for the set $\{y \in X: g(y)=0\}$, then we can choose for each $n \in \mathbb{N}$ a set $B\left(x, r_{n}\right) \backslash A_{x, 1 / n}$ as in Proposition 4.2 such that $u$ is $1 / n$-Lipschitz on 
$B\left(x, r_{n}\right) \backslash A_{x, 1 / n}$. Thus the limit function $u_{\infty}$ is $1 / n$-Lipschitz continuous for each $n \in \mathbb{N}$, and so is 0 -Lipschitz, that is, $u_{\infty}$ is constant.

The focus of the next section will be to study asymptotic behavior of the characteristic function $\chi_{E}$ of a set $E$ of finite perimeter at a boundary point. In considering such behavior, it is not possible to obtain a fruitful notion of the asymptotic limit of $\chi_{E}$ in a manner analogous to the above. Instead of considering a sequence of scaled versions of $\chi_{E}$, as with the scaled versions $u_{n}=[u-u(x)] / r_{n}$ above, we consider the scaled versions of the measures $\mu_{E}$ given by $d \mu_{E, n}:=\mu\left(B\left(x, r_{n}\right)\right)^{-1} \chi_{E} d \mu$, and study weak* limits of such measures. The rest of this section discusses how the two notions, one dealing with a scaled version of the function and the other with a scaled version of the measure, are related.

We fix a sequence $X_{n}=\left(X, d_{n}, x, \mu_{n}\right)$ that converges in the pointed measured Gromov-Hausdorff sense to a tangent space $X_{\infty}=\left(X_{\infty}, d_{\infty}, x_{\infty}, \mu_{\infty}\right)$ as discussed above, and for such a sequence we let $\nu_{n}$ be the measure on $X_{n}$ given by

$$
d \nu_{n}:=\mu\left(B\left(x, r_{n}\right)\right)^{-1}(u-u(x)) / r_{n} d \mu .
$$

We wish to show that the sequence of measures $\nu_{n}$ has a subsequence that converges to the measure $u_{\infty} d \mu_{\infty}$.

Theorem 4.10. Let $u \in B V(X)$. Then for $\mu$-a.e. $x \in X$ we have the following: if $\left(X_{\infty}, d_{\infty}, x_{\infty}, \mu_{\infty}\right)$ is any tangent space to $X$ at $x$, and $u_{\infty}$ is a function that arises as a limit adapted to $u$ at $x$, then also

$$
d \nu_{n}=\mu\left(B\left(x, r_{n}\right)\right)^{-1}(u-u(x)) / r_{n} d \mu \stackrel{*}{\rightarrow} u_{\infty} d \mu_{\infty} \quad \text { as } n \rightarrow \infty .
$$

Naturally, this weak limit is attained along the same subsequence as $u_{\infty}$ is. In addition to the connection that the above theorem makes between the way the limit function $u_{\infty}$ was obtained above and the tangent-space analysis of sets of finite perimeter in the next section, the theorem also gives an elegant way of constructing the limit function $u_{\infty}$ without having to modify the functions $\phi_{n}$ of Definition 3.1 to avoid the sets $A_{x}$.

Proof. Assume that $x$ is a Lebesgue point of $u$ such that

$$
\lim _{r \rightarrow 0} f_{B(x, r)}|g-g(x)| d \mu=0 \quad \text { and } \quad \lim _{r \rightarrow 0} \frac{V_{s}(u, B(x, r))}{\mu(B(x, r))}=0,
$$

and such that the conclusion of Lemma 4.3 holds. Let $A$ be the set of all points $y \in X$ such that for some $0<r \leq 2 d(x, y)$,

$$
f_{B(y, r)}|g-g(x)| d \mu+\frac{V_{s}(u, B(y, r))}{\mu(B(y, r))}>1 .
$$

Just as in the proof of Proposition 4.2, we get

$$
\lim _{r \rightarrow 0} \frac{\mu(A \cap B(x, r))}{\mu(B(x, r))}=0 .
$$

By Lemma 4.3 we obtain

$$
\lim _{r \rightarrow 0} \frac{1}{\mu(B(x, r))} \int_{A \cap B(x, r)} \frac{|u-u(x)|}{r} d \mu=0 .
$$


Fix $R>0$ and consider the embeddings $\iota: X_{\infty} \rightarrow Z$ and $\iota_{n}: X_{n} \rightarrow Z$. To prove the theorem, we need to show that whenever $\phi$ is a continuous function supported in $B_{Z}\left(\iota\left(x_{\infty}\right), R\right)$, we have

$$
\lim _{n \rightarrow \infty} \int_{Z} \phi \iota_{n, *}\left(d \nu_{n}\right)=\int_{Z} \phi \iota_{*}\left(u_{\infty} d \mu_{\infty}\right)
$$

Just as in Proposition 4.2, we have that for all sufficiently large $n \in \mathbb{N},\left.u\right|_{B\left(x, 2 R r_{n}\right) \backslash A}$ is $C(g(x)+1)$ Lipschitz. Then, define $\widetilde{u}$ to be the McShane extension of $\left.u\right|_{B\left(x, 2 R r_{n}\right) \backslash A}$. Just as in Theorem 4.8, we see that $u_{\infty}$ is a limit function of $(\widetilde{u}-\widetilde{u}(x)) / r_{n}$ which are all $C[g(x)+1]$-Lipschitz, and the sequence of measures $\mu\left(B\left(x, r_{n}\right)\right)^{-1} r_{n}^{-1}(\widetilde{u}-\widetilde{u}(x)) d \mu$ also converges weakly* to $u_{\infty} d \mu_{\infty}$. See Definition 3.6 and the discussion following it. It thus suffices to show that if $f_{n}:=(\widetilde{u}-\widetilde{u}(x)) / r_{n}$ and $g_{n}:=$ $(u-u(x)) / r_{n}$, then we have that $\mu\left(B\left(x, r_{n}\right)\right)^{-1}\left(f_{n}-g_{n}\right) d \mu \stackrel{*}{\rightarrow} 0$.

Let $\phi$ be a continuous function supported in the ball $B_{Z}\left(\iota\left(x_{\infty}\right), R\right)$. Then for all sufficiently large $n$,

$$
\begin{aligned}
\left|\int_{Z} \phi \iota_{n, *}\left(\left(f_{n}-g_{n}\right) d \mu_{n}\right)\right| & =\left|\int_{X_{n}} \phi\left(\iota_{n}(y)\right)\left(f_{n}-g_{n}\right)(y) d \mu_{n}(y)\right| \\
& \leq \frac{\|\phi\|_{\infty}}{\mu\left(B\left(x, r_{n}\right)\right)} \int_{B\left(x, 2 R r_{n}\right)}\left|\frac{\widetilde{u}-\widetilde{u}(x)}{r_{n}}-\frac{u-u(x)}{r_{n}}\right| d \mu \\
& \leq \frac{\|\phi\|_{\infty}}{\mu\left(B\left(x, r_{n}\right)\right)} \int_{B\left(x, 2 R r_{n}\right) \cap A}\left|\frac{\widetilde{u}-\widetilde{u}(x)}{r_{n}}\right|+\left|\frac{u-u(x)}{r_{n}}\right| d \mu .
\end{aligned}
$$

The last line follows since $\widetilde{u}(x)=u(x)$ and $\widetilde{u}=u$ on $B\left(x, 2 R r_{n}\right) \backslash A$. The first term converges to zero since $|\widetilde{u}-\widetilde{u}(x)| / r_{n} \leq C(g(x)+1) R$ on $B\left(x, 2 R r_{n}\right)$ by the Lipschitz bound for $\widetilde{u}$ and (4.7). Finally, the second term converges to zero by (4.8).

\section{Asymptotic Limits of SETS OF FINite PERIMEter}

Let $E \subset X$ be a set of finite perimeter, and fix a point $x \in \partial^{*} E$ such that Lemma 2.2 holds. We will zoom in at $x$ to study the asymptotic properties of $E$. Let $r_{n}>0$ with $r_{n} \rightarrow 0$. In this section, we always consider the sequence

$$
\left(X_{n}, d_{n}, x, \mu_{n}\right):=\left(X, \frac{1}{r_{n}} \cdot d, x, \frac{1}{\mu\left(B\left(x, r_{n}\right)\right)} \cdot \mu\right)
$$

under pointed measured Gromov-Hausdorff convergence. We also wish to study the behavior of the measure $P(E, \cdot)$ as we zoom in, so let

$$
\left(X_{n}, d_{n}, x, P_{n}(E, \cdot)\right):=\left(X, \frac{1}{r_{n}} \cdot d, x, \frac{r_{n}}{\mu\left(B\left(x, r_{n}\right)\right)} P(E, \cdot)\right) .
$$

Taking subsequences as necessary (not relabeled), we find the following measures on the limit space $\left(X_{\infty}, d_{\infty}, x_{\infty}\right)$ :

$$
\begin{gathered}
\mu_{n} \stackrel{*}{\rightarrow} \mu_{\infty}, \\
\mu_{n}(\cdot \cap E) \stackrel{*}{\rightarrow} \mu_{\infty}^{E}, \\
\mu_{n}\left(\cdot \cap E^{c}\right) \stackrel{*}{\rightarrow} \mu_{\infty}^{E^{c}}, \\
\text { and } P_{n}(E, \cdot) \stackrel{*}{\rightarrow} \pi_{\infty} .
\end{gathered}
$$

For ease of notation, we denote the ball $B_{X_{n}}(z, \rho)$ by $B_{n}(z, \rho)$; note that as a set, this is the same as the ball $B\left(z, r_{n} \rho\right)$ in $X$. In Section 3 it was noted that a tangent space $\left(X_{\infty}, d_{\infty}, x, \mu_{\infty}\right)$ always exists, is geodesic, and $\mu_{\infty}$ is doubling and supports a 1-Poincaré inequality. Note that 
$\mu_{n}(\cdot \cap E)+\mu_{n}\left(\cdot \cap E^{c}\right)=\mu_{n}$, and so $\mu_{\infty}^{E}+\mu_{\infty}^{E^{c}}=\mu_{\infty}$. The existence of $\pi_{\infty}$ follows from Lemma 2.2: by $(2.13)$, for every $k \in \mathbb{N}$,

$$
\limsup _{n \rightarrow \infty} P_{n}\left(E, B_{n}(x, k)\right)=\limsup _{n \rightarrow \infty} \frac{r_{n}}{\mu\left(B\left(x, r_{n}\right)\right)} P\left(E, B\left(x, k r_{n}\right)\right) \leq C_{d}^{1+\left\lceil\log _{2} k\right\rceil} .
$$

At various points in this section, we specify additional conditions on $x \in \partial^{*} E$ by excluding $\mathcal{H}$ negligible parts of $\partial^{*} E$.

Since $X$ is geodesic and $\mu$ is doubling, the space satisfies the following annular decay property: there exists $\delta=\delta\left(C_{d}\right) \in(0,1]$ such that for all $y \in X, r>0$, and $0<\varepsilon<1$, we have

$$
\mu(B(y, r) \backslash B(y, r(1-\varepsilon))) \leq C \varepsilon^{\delta} \mu(B(y, r)),
$$

see [9, Corollary 2.2]. In particular, this property implies that all spheres have zero $\mu$-measure.

We now define two sets in $X_{\infty}$. Let $(E)_{\infty}$ be the collection of all points $z \in X_{\infty}$ for which

$$
\lim _{r \rightarrow 0} \frac{\mu_{\infty}^{E}\left(B_{X_{\infty}}(z, r)\right)}{\mu_{\infty}\left(B_{X_{\infty}}(z, r)\right)}=1
$$

and let $\left(E^{c}\right)_{\infty}$ be the analogous collection of all points $z \in X_{\infty}$ for which

$$
\lim _{r \rightarrow 0} \frac{\mu_{\infty}^{E^{c}}\left(B_{X_{\infty}}(z, r)\right)}{\mu_{\infty}\left(B_{X_{\infty}}(z, r)\right)}=1 .
$$

Lemma 5.1. If $z \in X_{\infty}$ and $z_{n} \in X_{n}$ with $z_{n} \rightarrow z$ (or, more precisely, $\iota_{n}\left(z_{n}\right) \rightarrow \iota\left(z_{\infty}\right)$ in $Z$ ), then for every $r>0$,

$$
\mu_{\infty}\left(B_{X_{\infty}}(z, r)\right)=\lim _{n \rightarrow \infty} \mu_{n}\left(B_{n}\left(z_{n}, r\right)\right) .
$$

The analogous result holds for the measures $\mu_{n}(\cdot \cap E)$ and $\mu_{\infty}^{E}$, and for $\mu_{n}\left(\cdot \cap E^{c}\right)$ and $\mu_{\infty}^{E^{c}}$.

Proof. We prove the result for the measures $\mu_{n}(\cdot \cap E)$ and $\mu_{\infty}^{E}$; the proofs for the other two pairs are analogous. Fix $\eta>0$. By the lower semicontinuity of measure in open sets under weak* convergence (see e.g. [3, Proposition 1.62]),

$$
\mu_{\infty}^{E}\left(B_{X_{\infty}}(z, r-\eta)\right)=\mu_{\infty}^{E}\left(B_{Z}(z, r-\eta)\right) \leq \liminf _{n \rightarrow \infty}\left[\iota_{n, *} \mu_{n}(E \cap \cdot)\right]\left(B_{Z}(z, r-\eta)\right) .
$$

Here $B_{Z}(z, r-\eta)$ is the ball in $Z$ whose center is the image of $z$ under the isometric embedding $\iota$. Letting $\epsilon_{n}:=d_{Z}\left(z_{n}, z\right)$, we have $\epsilon_{n} \rightarrow 0$ and for large $n$,

$$
B_{Z}(z, r-\eta) \subset B_{Z}\left(z_{n}, r-\eta+\epsilon_{n}\right) \subset B_{Z}\left(z_{n}, r\right),
$$

where again we label the image of $z_{n}$ under the isometric embedding $\iota_{n}$ also by $z_{n}$. Now we can conclude

Thus letting $\eta \rightarrow 0$,

$$
\mu_{\infty}^{E}\left(B_{X_{\infty}}(z, r-\eta)\right) \leq \liminf _{n \rightarrow \infty} \mu_{n}\left(B_{n}\left(z_{n}, r\right) \cap E\right) .
$$

$$
\mu_{\infty}^{E}\left(B_{X_{\infty}}(z, r)\right) \leq \liminf _{n \rightarrow \infty} \mu_{n}\left(B_{n}\left(z_{n}, r\right) \cap E\right) .
$$

Again fix $\eta>0$. By upper semicontinuity of measure in compact sets under weak* convergence,

$$
\mu_{\infty}^{E}\left(B_{X_{\infty}}(z, r+2 \eta)\right) \geq \mu_{\infty}^{E}\left(\overline{B_{X_{\infty}}(z, r+\eta)}\right) \geq \limsup _{n \rightarrow \infty}\left[\iota_{n, *} \mu_{n}(E \cap \cdot)\right]\left(\overline{B_{Z}(z, r+\eta)}\right) .
$$

Again for large $n$,

$$
\left.\overline{B_{Z}(z, r+\eta)}\right) \supset B_{Z}\left(z_{n}, r+\eta-\epsilon_{n}\right) \supset B_{Z}\left(z_{n}, r\right)
$$


and we conclude

$$
\mu_{\infty}^{E}\left(B_{X_{\infty}}(z, r+2 \eta)\right) \geq \limsup _{n \rightarrow \infty} \mu_{n}\left(B_{n}\left(z_{n}, r\right) \cap E\right) .
$$

Note that the measure $\mu_{\infty}$ also satisfies the annular decay property, and that $\mu_{\infty}^{E} \leq \mu_{\infty}$, so spheres in $X_{\infty}$ do not carry positive $\mu_{\infty}^{E}$-weight; therefore letting $\eta \rightarrow 0$, we get

$$
\mu_{\infty}^{E}\left(B_{X_{\infty}}(z, r)\right) \geq \limsup _{n \rightarrow \infty} \mu_{n}\left(B_{n}\left(z_{n}, r\right) \cap E\right) .
$$

Combining this with (5.3) completes the proof.

Proposition 5.2. The sets $(E)_{\infty}$ and $\left(E^{c}\right)_{\infty}$ are disjoint. Moreover,

$$
\mu_{\infty}\left(X_{\infty} \backslash\left[(E)_{\infty} \cup\left(E^{c}\right)_{\infty}\right]\right)=0 .
$$

Proof. Suppose $z \in(E)_{\infty} \cap\left(E^{c}\right)_{\infty}$. Then

$$
\lim _{r \rightarrow 0} \frac{\mu_{\infty}^{E}\left(B_{X_{\infty}}(z, r)\right)}{\mu_{\infty}\left(B_{X_{\infty}}(z, r)\right)}=1=\lim _{r \rightarrow 0} \frac{\mu_{\infty}^{E^{c}}\left(B_{X_{\infty}}(z, r)\right)}{\mu_{\infty}\left(B_{X_{\infty}}(z, r)\right)} .
$$

By the Gromov-Hausdorff convergence, there is a sequence $x_{n} \in X_{n}$ with $x_{n} \rightarrow z$. Thus for any small enough $r>0$, Lemma 5.1 gives

$$
2 / 3<\frac{\mu_{\infty}^{E}\left(B_{X_{\infty}}(z, r)\right)}{\mu_{\infty}\left(B_{X_{\infty}}(z, r)\right)}=\lim _{n \rightarrow \infty} \frac{\mu_{n}\left(B_{n}\left(x_{n}, r\right) \cap E\right)}{\mu_{n}\left(B_{n}\left(x_{n}, r\right)\right)}=\lim _{n \rightarrow \infty} \frac{\mu\left(B\left(x_{n}, r r_{n}\right) \cap E\right)}{\mu\left(B\left(x_{n}, r r_{n}\right)\right)}
$$

and similarly

$$
2 / 3<\frac{\mu_{\infty}^{E^{c}}\left(B_{X_{\infty}}(z, r)\right)}{\mu_{\infty}\left(B_{X_{\infty}}(z, r)\right)}=\lim _{n \rightarrow \infty} \frac{\mu\left(B\left(x_{n}, r r_{n}\right) \cap E^{c}\right)}{\mu\left(B\left(x_{n}, r r_{n}\right)\right)} .
$$

Adding together, we find for all small enough $r>0$ and large enough $n$

$$
4 / 3<\frac{\mu\left(B\left(x_{n}, r r_{n}\right)\right)}{\mu\left(B\left(x_{n}, r r_{n}\right)\right)},
$$

which is not possible. Therefore $(E)_{\infty}$ and $\left(E^{c}\right)_{\infty}$ are disjoint.

Next, we show that $\mu_{\infty}\left(X_{\infty} \backslash\left[(E)_{\infty} \cup\left(E^{c}\right)_{\infty}\right]\right)=0$. To this end, we will show that the RadonNikodym derivative of $\mu_{\infty}^{E}$ with respect to $\mu_{\infty}$ is $\mu_{\infty}$-a.e. either 1 or 0 . Let this Radon-Nikodym derivative be denoted by $\varphi$. Let $A_{0}:=\left\{z \in X_{\infty}: 0<\varphi(z)<1\right\}$, and suppose that $\mu_{\infty}\left(A_{0}\right)>0$. Then there is some $R>0$ and $0<\delta<1$ such that the set

$$
A:=\left\{z \in B_{X_{\infty}}\left(x_{\infty}, R\right): \delta<\varphi(z)<1-\delta \text { and } z \text { is a Lebesgue point of } \varphi\right\}
$$

satisfies

$$
\mu_{\infty}(A)>\delta \mu_{\infty}\left(B_{X_{\infty}}\left(x_{\infty}, R\right)\right) .
$$

We fix $0<\varepsilon<R$ and consider the family of balls $B_{X_{\infty}}(z, \rho), z \in A$ and $0<\rho<\varepsilon$, such that

$$
\delta<\frac{1}{\mu_{\infty}\left(B_{X_{\infty}}(z, \rho)\right)} \int_{B_{X_{\infty}}(z, \rho)} \varphi d \mu_{\infty}<1-\delta .
$$

As every $z \in A$ is a Lebesgue point of $\varphi$, the corresponding family of closed balls is a fine cover of $A$, and hence there is a pairwise disjoint subfamily $\left\{\overline{B_{i}}\right\}_{i=1}^{\infty}$ such that $\mu_{\infty}\left(A \backslash \bigcup_{i=1}^{\infty} \overline{B_{i}}\right)=0$ and then also $\mu_{\infty}\left(A \backslash \bigcup_{i=1}^{\infty} B_{i}\right)=0$, since spheres have $\mu_{\infty}$-measure zero. Now, observe that

$$
\delta \mu_{\infty}\left(B_{X_{\infty}}\left(x_{\infty}, R\right)\right)<\mu_{\infty}(A) \leq \sum_{i=1}^{\infty} \mu_{\infty}\left(B_{i}\right),
$$


and so we can find $N \in \mathbb{N}$ such that

$$
\delta \mu_{\infty}\left(B_{X_{\infty}}\left(x_{\infty}, R\right)\right)<\sum_{i=1}^{N} \mu_{\infty}\left(B_{i}\right) .
$$

Denote the center of each $B_{i}$ by $x^{i}$. By Lemma 5.1 we can find $j_{\varepsilon} \in \mathbb{N}$ such that whenever $n \geq j_{\varepsilon}$, there are points $x_{n}^{1}, \cdots, x_{n}^{N} \in X=X_{n}$ converging to $x^{1}, \ldots, x^{N}$ respectively such that

$$
\left(1-\delta^{2}\right) \mu_{\infty}\left(B_{i}\right) \leq \mu_{n}\left(B_{n}\left(x_{n}^{i}, \operatorname{rad} B_{i}\right)\right) \leq\left(1+\delta^{2}\right) \mu_{\infty}\left(B_{i}\right) .
$$

for all $i=1, \ldots, N$. This gives

$$
\delta \frac{1-\delta^{2}}{1+\delta^{2}} \leq \frac{1-\delta^{2}}{1+\delta^{2}} \frac{1}{\mu_{\infty}\left(B_{i}\right)} \int_{B_{i}} \varphi d \mu_{\infty} \leq \frac{\mu_{n}\left(B_{n}\left(x_{n}^{i}, \operatorname{rad} B_{i}\right) \cap E\right)}{\mu_{n}\left(B_{n}\left(x_{n}^{i}, \operatorname{rad} B_{i}\right)\right)}
$$

$$
\leq \frac{1+\delta^{2}}{1-\delta^{2}} \frac{1}{\mu_{\infty}\left(B_{i}\right)} \int_{B_{i}} \varphi d \mu_{\infty} \leq \frac{1+\delta^{2}}{1+\delta} \leq 1-\delta / 2
$$

here, to obtain (5.8) we also used (5.4). We can also ensure that the collection of balls ("lifts" of $B_{i}$ to $\left.X_{n}\right)\left\{B\left(x_{n}^{i}, r_{n} \operatorname{rad} B_{i}\right)\right\}_{i=1}^{N}$ are pairwise disjoint. Inequality (5.8) tells us that if $\delta>0$ was chosen small enough, then

$$
\delta / 2 \leq \frac{\mu\left(B\left(x_{n}^{i}, r_{n} \operatorname{rad} B_{i}\right) \cap E\right)}{\mu\left(B\left(x_{n}^{i}, r_{n} \operatorname{rad} B_{i}\right)\right)} \leq 1-\delta / 2 .
$$

Now, applying the relative isoperimetric inequality (2.6) to these balls gives

$$
\delta / 2 \leq 2 C_{P} \frac{r_{n} \operatorname{rad} B_{i}}{\mu\left(B\left(x_{n}^{i}, r_{n} \operatorname{rad} B_{i}\right)\right)} P\left(E, B\left(x_{n}^{i}, r_{n} \operatorname{rad} B_{i}\right)\right) .
$$

Thus we obtain, recalling that $\operatorname{rad} B_{i}<\varepsilon<R$,

$$
\delta \sum_{i=1}^{N} \mu\left(B\left(x_{n}^{i}, r_{n} \operatorname{rad} B_{i}\right)\right) \leq 4 C_{P} \varepsilon r_{n} \sum_{i=1}^{N} P\left(E, B\left(x_{n}^{i}, r_{n} \operatorname{rad} B_{i}\right)\right) \leq 4 C_{P} \varepsilon r_{n} P\left(E, B\left(x, 2 r_{n} R\right)\right) .
$$

By (5.5) and (5.6), we now have

$$
\begin{aligned}
\delta^{2}\left(1-\delta^{2}\right) \mu_{\infty}\left(B_{X_{\infty}}\left(x_{\infty}, R\right)\right) & \leq \delta\left(1-\delta^{2}\right) \sum_{i=1}^{N} \mu_{\infty}\left(B_{i}\right) \\
& \leq \delta \sum_{i=1}^{N} \frac{\mu\left(B_{n}\left(x_{n}^{i}, r_{n} \operatorname{rad} B_{i}\right)\right)}{\mu\left(B\left(x, r_{n}\right)\right)} \\
& \leq \frac{4 C_{P} \varepsilon r_{n}}{\mu\left(B\left(x, r_{n}\right)\right)} P\left(E, B\left(x, 2 r_{n} R\right)\right) .
\end{aligned}
$$

Applying (5.7) now gives

$$
\frac{\delta^{2}\left(1-\delta^{2}\right)}{1+\delta^{2}} \frac{\mu\left(B\left(x, r_{n} R\right)\right)}{\mu\left(B\left(x, r_{n}\right)\right)} \leq \frac{4 C_{P} \varepsilon r_{n}}{\mu\left(B\left(x, r_{n}\right)\right)} P\left(E, B\left(x, 2 r_{n} R\right)\right) .
$$

By the doubling property of $\mu$ we obtain

$$
0<\frac{\delta^{2}\left(1-\delta^{2}\right)}{1+\delta^{2}} \frac{1}{4 C_{P} C_{d} \varepsilon} \leq \frac{r_{n}}{\mu\left(B\left(x, 2 r_{n} R\right)\right)} P\left(E, B\left(x, 2 r_{n} R\right)\right) .
$$


Now letting $n \rightarrow \infty$, by (2.13) we get

$$
0<\frac{\delta^{2}\left(1-\delta^{2}\right)}{1+\delta^{2}} \frac{1}{4 C_{P} C_{d} \varepsilon} \leq C_{d}
$$

for every $0<\varepsilon<1$, which is not possible. Thus $\mu\left(A_{0}\right)=0$.

Now the claim $\mu_{\infty}\left(X_{\infty} \backslash\left[(E)_{\infty} \cup\left(E^{c}\right)_{\infty}\right]\right)=0$ follows from the fact that $\mu_{\infty}^{E}+\mu_{\infty}^{E^{c}}=\mu_{\infty}$.

Note that by the above proposition and by the Radon-Nikodym theorem, we now have

$$
\mu_{\infty}^{E}(A)=\mu_{\infty}\left((E)_{\infty} \cap A\right) \quad \text { and } \quad \mu_{\infty}^{E^{c}}(A)=\mu_{\infty}\left(\left(E^{c}\right)_{\infty} \cap A\right)
$$

for Borel sets $A \subset X_{\infty}$, and moreover $\partial^{*}(E)_{\infty}=X_{\infty} \backslash\left((E)_{\infty} \cup\left(E^{c}\right)_{\infty}\right)$.

Now we wish to study the support of the asymptotic perimeter measure $\pi_{\infty}$ in $\left(X_{\infty}, d_{\infty}, x_{\infty}\right)$. We first prove a proposition that states that if a point $z \in X_{\infty}$ is in the support of $\pi_{\infty}$, then it can be seen as the limit of special points in $\partial^{*} E$. Recall from $(2.11)$ that

$$
\Sigma_{\gamma} E:=\left\{y \in X: \liminf _{r \rightarrow 0} \min \left\{\frac{\mu(B(y, r) \cap E)}{\mu(B(y, r))}, \frac{\mu\left(B(y, r) \cap E^{c}\right)}{\mu(B(y, r))}\right\} \geq \gamma\right\} \subset \partial^{*} E
$$

for some $\gamma=\gamma\left(C_{d}, C_{P}\right) \in(0,1 / 2]$. By $(2.10)$ and $(2.12)$ we know that $P(E, \cdot)$ is concentrated on $\Sigma_{\gamma} E$.

For each $m \in \mathbb{N}$, let

$$
G_{m}:=\left\{z \in \Sigma_{\gamma} E: \frac{\gamma}{2 C_{P}} \leq r \frac{P(E, B(z, r))}{\mu(B(z, r))} \leq 2 C_{d} \text { for all } 0<r<\frac{1}{m}\right\} .
$$

By Lemma 2.2 we know that

$$
\mathcal{H}\left(\partial^{*} E \backslash \bigcup_{m \in \mathbb{N}} G_{m}\right)=0,
$$

and $G_{m} \subset G_{m+1}$ for all $m \in \mathbb{N}$. Note that for every $r>0$ the map $z \mapsto P(E, B(z, r))$ is lower semicontinuous, and so $G_{m}$ is a Borel set. Combining the definitions of $\Sigma_{\gamma} E$ and $G_{m}$, for every $z \in \Sigma_{\gamma} E \cap G_{m}$ we find $r_{z}>0$ such that

$$
r P(E, B(z, r)) \leq K \min \left\{\mu(B(z, r) \cap E), \mu\left(B(z, r) \cap E^{c}\right)\right\}
$$

for all $0<r \leq r_{z}$, where $K=K\left(C_{d}, C_{P}\right)$. Hence we can refine $G_{m}$ further by considering the set

$$
G_{m}^{*}:=\left\{z \in G_{m}:(5.9) \text { holds for all } 0<r<\frac{1}{m}\right\} .
$$

Note then that $G_{m}^{*}$ is a Borel set, and that $G_{m}^{*} \subset G_{m+1}^{*}$ for $m \in \mathbb{N}$ with

$$
\mathcal{H}\left(\partial^{*} E \backslash \bigcup_{m \in \mathbb{N}} G_{m}^{*}\right)=0 .
$$

As $P(E, \cdot)$ is asymptotically doubling by $(2.13)$, we know that the Lebesgue differentiation theorem holds for the measure $P(E, \cdot)$. Hence for any fixed $m \in \mathbb{N}$, by the Lebesgue differentiation theorem, $G_{m}^{*}$ is of density 1 (with respect to the measure $P(E, \cdot)$ ) at $P(E, \cdot)$-a.e. $x \in G_{m}^{*}$. It is at such a point that we will zoom in and take our limiting measures.

Proposition 5.3. Let $\left(X_{n}, d_{n}, x, \mu_{n}\right)$ be a pointed measured Gromov-Hausdorff convergent sequence such that the base point $x$ is a point of $P(E, \cdot)$-density 1 for $G_{m}^{*}$ for some $m \in \mathbb{N}$. Suppose that $z \in X_{\infty}$ is such that

$$
\pi_{\infty}\left(B_{X_{\infty}}(z, R)\right)>0
$$


for all $R>0$. Then there is a sequence $z_{n} \in X_{n}$ that converges to $z$ (in $Z$ ) such that each $z_{n}$ is in $G_{m}^{*}$.

Proof. Fix $R>0$. By the Gromov-Hausdorff convergence there exist sequences $\epsilon_{n} \rightarrow 0$ and $x_{n} \in X_{n}=X$ such that $d_{Z}\left(x_{n}, z\right)<\epsilon_{n}$, and then by lower semicontinuity under weak* convergence, $(5.10)$

$$
\liminf _{n \rightarrow \infty} r_{n} \frac{P\left(E, B\left(x_{n},\left(R+\epsilon_{n}\right) r_{n}\right)\right)}{\mu\left(B\left(x, r_{n}\right)\right)} \geq \liminf _{n \rightarrow \infty} r_{n} \frac{P\left(E, \iota_{n}^{-1}\left(B_{Z}(z, R)\right)\right)}{\mu\left(B\left(x, r_{n}\right)\right)} \geq \pi_{\infty}\left(B_{X_{\infty}}(z, R)\right)>0 .
$$

We would like to know that there exists $\widetilde{x}_{n} \in G_{m}^{*} \cap B\left(x_{n},\left(R+\epsilon_{n}\right) r_{n}\right)$ for all sufficiently large $n \in \mathbb{N}$. Suppose that this is not the case. Then there is a subsequence $n_{k}$ such that $B\left(x_{n_{k}},\left(R+\epsilon_{n_{k}}\right) r_{n_{k}}\right)$ is disjoint from $G_{m}^{*}$. Choose $M>0$ large enough so that for all $k \in \mathbb{N}$,

$$
B\left(x_{n_{k}},\left(R+\epsilon_{n_{k}}\right) r_{n_{k}}\right) \subset B\left(x, M r_{n_{k}}\right) .
$$

As $B\left(x_{n_{k}},\left(R+\epsilon_{n_{k}}\right) r_{n_{k}}\right)$ and $B\left(x, M r_{n_{k}}\right) \cap G_{m}^{*}$ are disjoint, we have

$$
P\left(E, B\left(x_{n_{k}},\left(R+\epsilon_{n_{k}}\right) r_{n_{k}}\right)\right)+P\left(E, B\left(x, M r_{n_{k}}\right) \cap G_{m}^{*}\right) \leq P\left(E, B\left(x, M r_{n_{k}}\right)\right),
$$

which is equivalent to

$$
r_{n_{k}} \frac{P\left(E, B\left(x_{n_{k}},\left(R+\epsilon_{n_{k}}\right) r_{n_{k}}\right)\right)}{\mu\left(B\left(x, r_{n_{k}}\right)\right)}+r_{n_{k}} \frac{P\left(E, B\left(x, M r_{n_{k}}\right) \cap G_{m}^{*}\right)}{\mu\left(B\left(x, r_{n_{k}}\right)\right)} \leq r_{n_{k}} \frac{P\left(E, B\left(x, M r_{n_{k}}\right)\right)}{\mu\left(B\left(x, r_{n_{k}}\right)\right)} .
$$

Call the left-hand side of this inequality $A_{k}+B_{k}$, and the right-hand side $C_{k}$. The assumption that $x$ is a point of density 1 in $G_{m}^{*}$ implies that $B_{k} / C_{k} \rightarrow 1$ as $k \rightarrow \infty$. Thus $A_{k} / C_{k} \rightarrow 0$ as $k \rightarrow \infty$. On the other hand, by the definition of $G_{m}$, we must have $C_{k} \leq 2 M^{-1} C_{d}^{2+\log _{2}(M)}<\infty$ for large $k \in \mathbb{N}$. Therefore $A_{k} \rightarrow 0$ as $k \rightarrow \infty$, which contradicts (5.10). Thus, there is some $N_{1} \in \mathbb{N}$ such that there is a point $\widetilde{x}_{n} \in G_{m}^{*} \cap B\left(x_{n},\left(R+\epsilon_{n}\right) r_{n}\right)$ for all $n \geq N_{1}$. We rename this sequence $\widetilde{x}_{n}^{1}$. Similarly, there exists a sequence

$$
\widetilde{x}_{n}^{2} \in G_{m}^{*} \cap B\left(x_{n},\left(2^{-1} R+\epsilon_{n}\right) r_{n}\right)
$$

for all $n \geq N_{2}>N_{1}$. We continue inductively in this fashion to find for each $k \in \mathbb{N}$,

$$
\widetilde{x}_{n}^{k} \in G_{m}^{*} \cap B\left(x_{n},\left(2^{-k} R+\epsilon_{n}\right) r_{n}\right)
$$

for all $n \geq N_{k}>N_{k-1}$. Now

$$
d_{Z}\left(\widetilde{x}_{n}^{k}, z\right) \leq d_{Z}\left(\widetilde{x}_{n}^{k}, x_{n}\right)+d_{Z}\left(x_{n}, z\right) \leq 2^{-k} R+\epsilon_{n}+\epsilon_{n} .
$$

For $n \in\left[N_{k}, N_{k+1}\right)$, set $z_{n}:=\widetilde{x}_{n}^{k}$. Then $z_{n}$ has the desired properties.

Note also that the support of $\pi_{\infty}$ is contained in $X_{\infty}$; this can be seen as follows. If $z \in Z$ such that $\pi_{\infty}\left(B_{Z}(z, R)\right)>0$ for all $R>0$, then there exists a sequence $x_{n} \in X_{n}$ such that $x_{n} \rightarrow z$ in $Z$. It follows that $z \in X_{\infty}$.

We now provide growth estimates for the measure $\pi_{\infty}$.

Theorem 5.4. Consider the sequence $\left(X_{n}, d_{n}, x, \mu_{n}\right)$ such that $x$ is a point of $P(E, \cdot)$-density 1 for $G_{m}^{*}$ for some $m \in \mathbb{N}$. Suppose that $z \in X_{\infty}$ is such that

$$
\pi_{\infty}\left(B_{X_{\infty}}(z, R)\right)>0
$$

for all $R>0$. Then

$$
\frac{1}{C} \frac{\mu_{\infty}\left(B_{X_{\infty}}(z, r)\right)}{r} \leq \pi_{\infty}\left(B_{X_{\infty}}(z, r)\right) \leq C \frac{\mu_{\infty}\left(B_{X_{\infty}}(z, r)\right)}{r} \quad \text { for all } r>0
$$


where $C=C\left(C_{d}, C_{P}\right)$, and

$$
\pi_{\infty}\left((E)_{\infty} \cup\left(E^{c}\right)_{\infty}\right)=0
$$

Proof. By Proposition 5.3, there is a sequence $z_{n} \in G_{m}^{*}$ that converges to $z$ in $Z$. Fix $r>0$ and $0<\eta<r / 2$. Using the basic properties of weak* convergence as in the proof of Proposition 5.3, we find a sequence of positive numbers $\epsilon_{n}$ with $\lim _{n \rightarrow \infty} \epsilon_{n}=0$ such that

$$
\liminf _{n \rightarrow \infty} r_{n} \frac{P\left(E, B\left(z_{n},\left(r+\epsilon_{n}\right) r_{n}\right)\right)}{\mu\left(B\left(x, r_{n}\right)\right)} \geq \pi_{\infty}\left(B_{X_{\infty}}(z, r)\right) \geq \limsup _{n \rightarrow \infty} r_{n} \frac{P\left(E, B\left(z_{n},(r-\eta) r_{n}\right)\right)}{\mu\left(B\left(x, r_{n}\right)\right)} .
$$

We can rewrite the term on the right-most side of (5.13) as

$$
\limsup _{n \rightarrow \infty}(r-\eta) r_{n} \frac{P\left(E, B\left(z_{n},(r-\eta) r_{n}\right)\right)}{\mu\left(B\left(z_{n},(r-\eta) r_{n}\right)\right)} \cdot \frac{\mu\left(B\left(z_{n},(r-\eta) r_{n}\right)\right)}{(r-\eta) \mu\left(B\left(x, r_{n}\right)\right)} .
$$

Since $z_{n} \in G_{m}^{*}$, we know that

$$
(r-\eta) r_{n} \frac{P\left(E, B\left(z_{n},(r-\eta) r_{n}\right)\right)}{\mu\left(B\left(z_{n},(r-\eta) r_{n}\right)\right)} \geq \frac{\gamma}{2 C_{P}}
$$

for all large enough $n$ (that is, when $(r-\eta) r_{n}<1 / m$ ). Additionally, by Lemma 5.1,

$$
\begin{aligned}
\limsup _{n \rightarrow \infty} \frac{\mu\left(B\left(z_{n},(r-\eta) r_{n}\right)\right)}{(r-\eta) \mu\left(B\left(x, r_{n}\right)\right)} & =\frac{1}{(r-\eta)} \limsup _{n \rightarrow \infty} \frac{\mu\left(B\left(z_{n},(r-\eta) r_{n}\right)\right)}{\mu\left(B\left(x, r_{n}\right)\right)} \\
& =\frac{\mu_{\infty}\left(B_{X_{\infty}}(z, r-\eta)\right)}{r-\eta} \\
& \geq \frac{1}{C_{d}^{2}} \frac{\mu_{\infty}\left(B_{X_{\infty}}(z, r)\right)}{r}
\end{aligned}
$$

since $\mu_{\infty}$ is doubling with constant $C_{d}^{2}$. Thus by (5.13), we get

$$
\pi_{\infty}\left(B_{X_{\infty}}(z, r)\right) \geq \frac{\gamma}{2 C_{d}^{2} C_{P}} \frac{\mu_{\infty}\left(B_{X_{\infty}}(z, r)\right)}{r} .
$$

Next we rewrite the term on the left-most side of (5.13) as

$$
\liminf _{n \rightarrow \infty}\left(r+\epsilon_{n}\right) r_{n} \frac{P\left(E, B\left(z_{n},\left(r+\epsilon_{n}\right) r_{n}\right)\right)}{\mu\left(B\left(z_{n},\left(r+\epsilon_{n}\right) r_{n}\right)\right)} \cdot \frac{\mu\left(B\left(z_{n},\left(r+\epsilon_{n}\right) r_{n}\right)\right)}{\left(r+\epsilon_{n}\right) \mu\left(B\left(x, r_{n}\right)\right)} .
$$

Since $z \in G_{m}^{*}$, we know that

$$
\left(r+\epsilon_{n}\right) r_{n} \frac{P\left(E, B\left(z_{n},\left(r+\epsilon_{n}\right) r_{n}\right)\right)}{\mu\left(B\left(z_{n},\left(r+\epsilon_{n}\right) r_{n}\right)\right)} \leq 2 C_{d}
$$

for all large enough $n$. Similarly to above, we obtain

$$
\lim _{n \rightarrow \infty} \frac{\mu\left(B\left(z_{n},\left(r+\epsilon_{n}\right) r_{n}\right)\right)}{\left(r+\epsilon_{n}\right) \mu\left(B\left(x, r_{n}\right)\right)}=\frac{\mu_{\infty}\left(B_{X_{\infty}}(z, r)\right)}{r},
$$

whence from (5.13) we obtain

$$
\pi_{\infty}\left(B_{X_{\infty}}(z, r)\right) \leq 2 C_{d} \frac{\mu_{\infty}\left(B_{X_{\infty}}(z, r)\right)}{r} .
$$

This proves (5.11).

It now only remains to show (5.12). It suffices to show that when $z \in(E)_{\infty} \cup\left(E^{c}\right)_{\infty}$, for each $k \in \mathbb{N}$ there is some $r_{z}>0$ such that $\pi_{\infty}\left(B_{X_{\infty}}\left(z, r_{z}\right)\right) \leq C \mu_{\infty}\left(B_{X_{\infty}}\left(z, r_{z}\right)\right) /\left(k r_{z}\right)$, from which we 
will know (via (5.11)) that there must be $\rho_{z}>0$ with $\pi_{\infty}\left(B_{X_{\infty}}\left(z, \rho_{z}\right)\right)=0$. Fix $k \in \mathbb{N}$. Suppose $z \in(E)_{\infty}$ is in the support of $\pi_{\infty}$. Then there is some $r_{z}>0$ such that

$$
\frac{\mu_{\infty}\left(B_{X_{\infty}}\left(z, r_{z}\right) \cap\left(E^{c}\right)_{\infty}\right)}{\mu_{\infty}\left(B_{X_{\infty}}\left(z, r_{z}\right)\right)}<\frac{1}{k} .
$$

Let $\epsilon_{n} \rightarrow 0$ and $G_{m}^{*} \ni z_{n} \rightarrow z$ as given by the conclusion of Proposition 5.3. By Lemma 5.1 we have

$$
\begin{aligned}
\frac{1}{k} \lim _{n \rightarrow \infty} \frac{\mu\left(B\left(z_{n}, r_{z} r_{n}\right)\right)}{\mu\left(B\left(x, r_{n}\right)\right)}=\frac{\mu_{\infty}\left(B_{X_{\infty}}\left(z, r_{z}\right)\right)}{k} & >\mu_{\infty}\left(B_{X_{\infty}}\left(z, r_{z}\right) \cap\left(E^{c}\right)_{\infty}\right) \\
& =\lim _{n \rightarrow \infty} \frac{\mu\left(B\left(z_{n}, r_{z} r_{n}\right) \cap E^{c}\right)}{\mu\left(B\left(x, r_{n}\right)\right)} .
\end{aligned}
$$

Thus for all large enough $n \in \mathbb{N}$,

$$
\frac{1}{k} \mu\left(B\left(z_{n}, r_{z} r_{n}\right)\right) \geq \mu\left(B\left(z_{n}, r_{z} r_{n}\right) \cap E^{c}\right) .
$$

Since $z_{n} \in G_{m}^{*}$, by (5.9) we have that for all large enough $n \in \mathbb{N}$,

$$
r_{z} r_{n} \frac{P\left(E, B\left(z_{n}, r_{z} r_{n}\right)\right)}{\mu\left(B\left(z_{n}, r_{z} r_{n}\right)\right)} \leq K \frac{\mu\left(B\left(z_{n}, r_{z} r_{n}\right) \cap E^{c}\right)}{\mu\left(B\left(z_{n}, r_{z} r_{n}\right)\right)} \leq \frac{K}{k} .
$$

Thus for any $\eta>0$,

$$
\begin{aligned}
\pi_{\infty}\left(B_{X_{\infty}}\left(z, r_{z}-\eta\right)\right) \leq \liminf _{n \rightarrow \infty} r_{n} \frac{P\left(E, B\left(z_{n}, r_{z} r_{n}\right)\right)}{\mu\left(B\left(x, r_{n}\right)\right)} & \leq \frac{K}{k} \frac{1}{r_{z}} \liminf _{n \rightarrow \infty} \frac{\mu\left(B\left(z_{n}, r_{z} r_{n}\right)\right)}{\mu\left(B\left(x, r_{n}\right)\right)} \\
& =\frac{K}{k} \frac{\mu_{\infty}\left(B_{X_{\infty}}\left(z, r_{z}\right)\right)}{r_{z}}
\end{aligned}
$$

by Lemma 5.1. Since $K=K\left(C_{d}, C_{P}\right)$, letting $\eta \rightarrow 0$ gives

$$
\pi_{\infty}\left(B_{X_{\infty}}\left(z, r_{z}\right)\right) \leq \frac{K}{k} \frac{\mu_{\infty}\left(B_{X_{\infty}}\left(z, r_{z}\right)\right)}{r_{z}} .
$$

By choosing $k \in \mathbb{N}$ large enough, the above would violate the left-hand inequality of (5.11), and so $z$ cannot be in the support of $\pi_{\infty}$. Thus, there is some $\rho_{z}>0$ with $\pi_{\infty}\left(B\left(z, \rho_{z}\right)\right)=0$. Since this happens for every $z \in(E)_{\infty}$, we know that $\pi_{\infty}$ does not charge $(E)_{\infty}$. Indeed, with

$$
(E)_{\infty} \subset U:=\bigcup_{z \in(E)_{\infty}} B\left(z, \rho_{z}\right)
$$

an open set containing $(E)_{\infty}$, we have $\pi_{\infty}(U)=0$. A similar argument gives the existence of an open set $V \supset\left(E^{c}\right)_{\infty}$ with $\pi_{\infty}(V)=0$. This completes the proof.

Next we show that the set $(E)_{\infty}$ is of locally finite perimeter in the space $X_{\infty}$. Denote by $\mathcal{H}$ the co-dimension 1 Hausdorff measure in the space $\left(X_{\infty}, d_{\infty}, \mu_{\infty}\right)$.

Theorem 5.5. For all $R>0$, we have $P\left((E)_{\infty}, B_{X_{\infty}}\left(x_{\infty}, R\right)\right)<\infty$. The measures $P\left((E)_{\infty}, \cdot\right)$, $\pi_{\infty}$, and $\mathcal{H}\left(\partial^{*}(E)_{\infty} \cap \cdot\right)$ are comparable. The sets $(E)_{\infty}$ and $\left(E^{c}\right)_{\infty}$ are open in $X_{\infty}$.

Proof. To prove the first claim, we use a discrete convolution construction. Assume for simplicity that $R=1$. Fix $0<\epsilon<1 / 9$, and take a maximal $\epsilon$-separated set $\left\{z_{k}\right\}_{k=1}^{\infty} \subset X_{\infty}$. Then the balls $B_{k}:=B_{X_{\infty}}\left(z_{k}, \epsilon\right)$ cover $X_{\infty}$ and $B_{X_{\infty}}\left(z_{k}, 14 \varepsilon\right)$ have bounded overlap. For each $k$ we can find points 
$z_{k, n} \in X_{n}$ converging to $z_{k}$ (in $Z$ ). Thus, by considering a tail-end of the sequence if necessary, there is a sequence $0<\delta_{n} \rightarrow 0$ with $\delta_{n}<\epsilon$ such that $d_{Z}\left(\iota_{\infty}\left(z_{k}\right), \iota_{n}\left(z_{k, n}\right)\right)<\delta_{n}$, and by Lemma 5.1,

$$
\left(1-\delta_{n}\right) \mu_{\infty}^{E}\left(B_{X_{\infty}}\left(z_{k}, \epsilon\right)\right) \leq \mu_{n}\left(B_{n}\left(z_{k, n}, \epsilon\right) \cap E\right)
$$

and

$$
\mu_{n}\left(B_{n}\left(z_{k, n}, \epsilon\right)\right) \leq\left(1+\delta_{n}\right) \mu_{\infty}\left(B_{X_{\infty}}\left(z_{k}, \epsilon\right)\right) \leq\left(1+\delta_{n}\right)^{2} \mu_{n}\left(B_{n}\left(z_{k, n}, \epsilon\right)\right) .
$$

Observe that we need only do this for the points $z_{k} \in B_{X_{\infty}}\left(x_{\infty}, 2\right)$, of which there are only finitely many, and thus we can choose $\delta_{n}>0$ such that the above hold for all corresponding indices $k$. By the bounded overlap property of the balls $B_{X_{\infty}}\left(z_{k}, 14 \epsilon\right)$, we also have that for each such positive integer $n$, the collection of balls $B_{n}\left(z_{k, n}, 6 \epsilon\right)$ has a bounded overlap; this will be needed in the computations (5.18).

Now take a partition of unity by means of $C / \epsilon$-Lipschitz functions $\phi_{k} \in \operatorname{Lip}\left(X_{\infty} ;[0,1]\right)$ with $\operatorname{supp}\left(\phi_{k}\right) \subset B_{X_{\infty}}\left(z_{k}, 2 \epsilon\right)$ for each $k \in \mathbb{N}$; see e.g. [21, p. 104]. Let $u:=\chi_{E}$, and for each $n \in \mathbb{N}$ we set

$$
v_{n}^{\epsilon}:=\sum_{k=1}^{\infty} u_{B_{n}\left(z_{k, n}, \epsilon\right)} \phi_{k},
$$

where

$$
u_{B_{n}\left(z_{k, n}, \epsilon\right)}=f_{B_{n}\left(z_{k, n}, \epsilon\right)} u d \mu_{n}=f_{B\left(z_{k, n}, r_{n} \epsilon\right)} u d \mu=\frac{\mu\left(B\left(z_{k, n}, r_{n} \epsilon\right) \cap E\right)}{\mu\left(B\left(z_{k, n}, r_{n} \epsilon\right)\right)} .
$$

Let $l \in \mathbb{N}$ such that $B_{l} \cap B_{X_{\infty}}\left(x_{\infty}, 1\right) \neq \emptyset$. Given $y_{1}, y_{2} \in B_{l}$, we estimate

$$
\begin{aligned}
\left|v_{n}^{\epsilon}\left(y_{1}\right)-v_{n}^{\epsilon}\left(y_{2}\right)\right|= & \left|\sum_{k=1}^{\infty} u_{B_{n}\left(z_{k, n}, \epsilon\right)} \phi_{k}\left(y_{1}\right)-\sum_{k=1}^{\infty} u_{B_{n}\left(z_{k, n}, \epsilon\right)} \phi_{k}\left(y_{2}\right)\right| \\
= & \left|\sum_{k=1}^{\infty}\left(u_{B_{n}\left(z_{k, n}, \epsilon\right)}-u_{B_{n}\left(z_{l, n}, \epsilon\right)}\right)\left(\phi_{k}\left(y_{1}\right)-\phi_{k}\left(y_{2}\right)\right)\right| \\
\leq & \left.\sum_{k=1}^{\infty} \mid u_{B_{n}\left(z_{k, n}, \epsilon\right)}-u_{B_{n}\left(z_{l, n}, \epsilon\right)}\right)\left|\phi_{k}\left(y_{1}\right)-\phi_{k}\left(y_{2}\right)\right| \\
= & \sum_{\substack{k \in \mathbb{N} \\
B_{\infty}\left(z_{k}, 2 \epsilon\right) \cap B_{l} \neq \emptyset}}\left|u_{B_{n}\left(z_{k, n}, \epsilon\right)}-u_{B_{n}\left(z_{l, n}, \epsilon\right)}\right|\left|\phi_{k}\left(y_{1}\right)-\phi_{k}\left(y_{2}\right)\right| \\
\leq & C \sum_{\substack{k \in \mathbb{N} \\
B_{X_{\infty}}\left(z_{k}, 2 \epsilon\right) \cap B_{l} \neq \emptyset}}\left|u_{B_{n}\left(z_{k, n}, \epsilon\right)}-u_{B_{n}\left(z_{l, n}, \epsilon\right)}\right| \frac{d_{\infty}\left(y_{1}, y_{2}\right)}{\epsilon} .
\end{aligned}
$$

Note that for the indices $k$ in the last sum, we have $d_{\infty}\left(z_{k}, z_{l}\right) \leq 3 \epsilon$ and so $d_{n}\left(z_{k, n}, z_{l, n}\right) \leq 5 \epsilon$. Thus, each ball $B_{n}\left(z_{k, n}, \epsilon\right)$ is contained in $B_{n}\left(z_{l, n}, 6 \epsilon\right)$. By the bounded overlap property, there are at most $J$ number of balls $B_{n}\left(z_{k, n}, \epsilon\right)$ contained in $B_{n}\left(z_{l, n}, 6 \epsilon\right)$, with $J$ depending solely on the doubling constant of $\mu_{n}$ - which is the same doubling constant of $\mu$. Moreover, for each $k$ for which $B_{X_{\infty}}\left(z_{k}, 2 \epsilon\right) \cap B_{l} \neq \emptyset$, we have that

$$
\left|u_{B_{n}\left(z_{k, n}, \epsilon\right)}-u_{B_{n}\left(z_{l, n}, \epsilon\right)}\right| \leq C f_{B_{n}\left(z_{l, n}, 6 \epsilon\right)}\left|u-u_{B_{n}\left(z_{l, n}, 6 \epsilon\right)}\right| d \mu_{n} .
$$


Thus, we can continue the estimate via the Poincaré inequality:

$$
\begin{aligned}
\left|v_{n}^{\epsilon}\left(y_{1}\right)-v_{n}^{\epsilon}\left(y_{2}\right)\right| & \leq C f_{B_{n}\left(z_{l, n}, 6 \epsilon\right)}\left|u-u_{B_{n}\left(z_{l, n}, 6 \epsilon\right)}\right| d \mu_{n} \frac{d_{\infty}\left(y_{1}, y_{2}\right)}{\epsilon} \\
& \leq C \epsilon \frac{d_{\infty}\left(y_{1}, y_{2}\right)}{\epsilon} \frac{P_{n}\left(E, B_{n}\left(z_{l, n}, 6 \epsilon\right)\right)}{\mu_{n}\left(B_{n}\left(z_{l, n}, 6 \epsilon\right)\right)} .
\end{aligned}
$$

Thus, we get for $y \in B_{l}$,

$$
\operatorname{Lip} v_{n}^{\epsilon}(y) \leq C \frac{P_{n}\left(E, B_{n}\left(z_{l, n}, 6 \epsilon\right)\right)}{\mu_{n}\left(B_{n}\left(z_{l, n}, 6 \epsilon\right)\right)} .
$$

Therefore, in $B_{X_{\infty}}\left(x_{\infty}, 1\right)$,

$$
\operatorname{Lip} v_{n}^{\epsilon} \leq C \sum_{l=1}^{\infty} \chi_{B_{l}} \frac{P_{n}\left(E, B_{n}\left(z_{l, n}, 6 \epsilon\right)\right)}{\mu_{n}\left(B_{n}\left(z_{l, n}, \epsilon\right)\right)} .
$$

By the definition of pointed measured Gromov-Hausdorff convergence, $\lim _{n \rightarrow \infty} d_{Z}\left(\iota_{n}(x), \iota\left(x_{\infty}\right)\right)=$ 0 , see Definition 3.3 and the discussion preceding it. If $B_{l} \cap B_{X_{\infty}}\left(x_{\infty}, 1\right) \neq \emptyset$, then $B_{n}\left(z_{l, n}, 2 \epsilon\right) \cap$ $B_{n}(x, 1+\varepsilon) \neq \emptyset$. Hence by $(5.15)$ and by the bounded overlap of the family $B_{n}\left(z_{k, n}, 6 \epsilon\right), k \in \mathbb{N}$, we have

$$
\begin{aligned}
\int_{B_{X_{\infty}}\left(x_{\infty}, 1\right)} \operatorname{Lip} v_{n}^{\epsilon} d \mu_{\infty} & \leq C \sum_{\substack{l \in \mathbb{N} \\
B_{n}\left(z_{l, n}, 2 \epsilon\right) \cap B_{n}(x, 1+\varepsilon) \neq \emptyset}} \mu_{\infty}\left(B_{l}\right) \frac{P_{n}\left(E, B_{n}\left(z_{l, n}, 6 \epsilon\right)\right)}{\mu_{n}\left(B_{n}\left(z_{l, n}, \epsilon\right)\right)} \\
& \leq C\left(1+\delta_{n}\right) \sum_{\substack{l \in \mathbb{N} \\
B_{n}\left(z_{l, n}, 2 \epsilon\right) \cap B_{n}(x, 1+\varepsilon) \neq \emptyset}} P_{n}\left(E, B_{n}\left(z_{l, n}, 6 \epsilon\right)\right) \\
& \leq C P_{n}\left(E, B_{n}(x, 1+9 \epsilon)\right) \\
& \leq C P_{n}\left(E, B_{n}(x, 2)\right) .
\end{aligned}
$$

This remains bounded as $n \rightarrow \infty$, see (5.1). We can do the above for a sequence $\epsilon_{i} \rightarrow 0$, with $n=n(i) \rightarrow \infty$ and $\delta_{n_{i}} \rightarrow 0$, to obtain a sequence of functions $v_{i}=v_{n(i)}^{\epsilon_{i}} \in \operatorname{Lip}\left(B_{X_{\infty}}\left(x_{\infty}, 1\right)\right)$. Since $V\left(v_{i},\left(B_{X_{\infty}}\left(x_{\infty}, 1\right)\right)\right.$ is bounded by $(5.18)$, we find a subsequence, also denoted by $v_{i}$, such that $v_{i} \rightarrow w$ in $L^{1}\left(B_{X_{\infty}}\left(x_{\infty}, 1\right)\right)$, see [33, Theorem 3.7]. By lower semicontinuity,

$$
V\left(w, B_{X_{\infty}}\left(x_{\infty}, 1\right)\right) \leq \liminf _{i \rightarrow \infty} \int_{B_{X_{\infty}}\left(x_{\infty}, 1\right)} \operatorname{Lip} v_{i} d \mu_{\infty} \leq C \limsup _{n \rightarrow \infty} P_{n}\left(B_{n}(x, 2)\right) \leq \pi_{\infty}\left(B_{X_{\infty}}\left(x_{\infty}, 3\right)\right)
$$

and so $w \in B V\left(B_{X_{\infty}}\left(x_{\infty}, 1\right)\right)$. We need to check that $w=\chi_{(E)_{\infty}}$ in $L^{1}\left(B_{X_{\infty}}\left(x_{\infty}, 1\right)\right)$. To do so, fix $y \in B_{X_{\infty}}\left(x_{\infty}, 1\right) \cap(E)_{\infty}$ and fix $\eta \in(0,1)$. Then by definition of $(E)_{\infty}$, for large enough $i \in \mathbb{N}$ we have

$$
\frac{\mu_{\infty}^{E}\left(B_{X_{\infty}}\left(y, 4 \epsilon_{i}\right)\right)}{\mu_{\infty}\left(B_{X_{\infty}}\left(y, 4 \epsilon_{i}\right)\right)} \geq 1-\eta
$$

We denote the covering of $X_{\infty}$ corresponding to an index $i \in \mathbb{N}$ by $B_{k}^{i}:=B\left(z_{k}^{i}, \epsilon_{i}\right)$. It follows that for all balls $B_{k}^{i}$ with $2 B_{k}^{i}$ containing $y$, we have (note that $\mu_{\infty}$ is doubling with constant $C_{d}^{2}$ )

$$
\frac{\mu_{\infty}^{E^{c}}\left(B_{X_{\infty}}\left(z_{k}^{i}, \epsilon_{i}\right)\right)}{\mu_{\infty}\left(B_{X_{\infty}}\left(z_{k}^{i}, \epsilon_{i}\right)\right)} \leq C_{d}^{6} \frac{\mu_{\infty}^{E^{c}}\left(B_{X_{\infty}}\left(y, 4 \epsilon_{i}\right)\right)}{\mu_{\infty}\left(B_{X_{\infty}}\left(y, 4 \epsilon_{i}\right)\right)} \leq C_{d}^{6} \eta
$$


Thus by (5.14) and (5.15),

$$
\frac{\mu_{n_{i}}\left(B_{n_{i}}\left(z_{k, n_{i}}, \epsilon_{i}\right) \cap E\right)}{\mu_{n_{i}}\left(B_{n_{i}}\left(z_{k, n_{i}}, \epsilon_{i}\right)\right)} \geq \frac{1-\delta_{n_{i}}}{1+\delta_{n_{i}}} \frac{\mu_{\infty}^{E}\left(B_{X_{\infty}}\left(z_{k}^{i}, \epsilon_{i}\right)\right)}{\mu_{\infty}\left(B_{X_{\infty}}\left(z_{k}^{i}, \epsilon_{i}\right)\right)} \geq \frac{1-\delta_{n_{i}}}{1+\delta_{n_{i}}}\left(1-C_{d}^{6} \eta\right) .
$$

Now, by definition of the discrete convolutions (5.16), we have

$$
v_{i}(y) \geq \frac{1-\delta_{n_{i}}}{1+\delta_{n_{i}}}\left(1-C_{d}^{6} \eta\right) .
$$

Letting $i \rightarrow \infty$, we get

$$
w(y) \geq 1-C_{d}^{6} \eta
$$

Since $\eta>0$ was arbitrary, we conclude $w(y)=1$ (the values taken on by the functions $v_{i}$ are between 0 and 1 , so necessarily $w(y) \leq 1)$. Similarly, we get $w(y)=0$ for all $y \in\left(E^{c}\right)_{\infty}$. Also by Proposition 5.2 we know that $\mu_{\infty}\left(X_{\infty} \backslash\left((E)_{\infty} \cup\left(E^{c}\right)_{\infty}\right)\right)=0$. Thus $w=\chi_{(E)_{\infty}}$ as functions in $L^{1}\left(B_{X_{\infty}}\left(x_{\infty}, 1\right)\right)$. Recall that we are assuming $R=1$ just for convenience; we conclude that $\chi_{(E)_{\infty}} \in B V\left(B_{X_{\infty}}\left(x_{\infty}, R\right)\right)$ for all $R>0$.

Next, for $z \in X_{\infty}$ and $r>0$, by an argument analogous to that leading to (5.19), we obtain

$$
P\left((E)_{\infty}, B(z, r)\right) \leq \pi_{\infty}(z, 3 r) .
$$

From the final part of the proof of Theorem 5.4, we know that $\pi_{\infty}$ is supported inside $X \backslash(U \cup V)$, where $U$ and $V$ are (open) neighborhoods of $(E)_{\infty}$ and $\left(E^{c}\right)_{\infty}$, respectively. Conversely, if $z \in$ $X_{\infty} \backslash\left[(E)_{\infty} \cup\left(E^{c}\right)_{\infty}\right]$, which we recall is the same set as $\partial^{*}(E)_{\infty}$, then $z$ is in the support of $P\left((E)_{\infty}, \cdot\right)$ by the relative isoperimetric inequality (2.6). Thus by (5.20), $z$ is in the support of $\pi_{\infty}$. In conclusion, the support of $\pi_{\infty}$ is exactly $\partial^{*}(E)_{\infty}=X_{\infty} \backslash[U \cup V]$. Moreover, by (5.11) and Lemma 2.1 we know that $\pi_{\infty}$ is comparable to $\mathcal{H}\left(\partial^{*}(E)_{\infty} \cap \cdot\right)$. By $(2.10)$ we know that $P\left((E)_{\infty}, \cdot\right)$ is also comparable to $\mathcal{H}\left(\partial^{*}(E)_{\infty} \cap \cdot\right)$. Thus the three measures $\pi_{\infty}, P\left((E)_{\infty}, \cdot\right)$, and $\mathcal{H}\left(\partial^{*}(E)_{\infty} \cap \cdot\right)$ are all comparable. Finally, by the relative isoperimetric inequality and the fact that $P\left((E)_{\infty}, \cdot\right)$ does not see the set $U$, it follows that $U$ cannot intersect $\left(E^{c}\right)_{\infty}$. Indeed, if $U \cap\left(E^{c}\right)_{\infty}$ is non-empty, then by the construction of $U$, we can find $z \in(E)_{\infty}$ and $\rho_{z}>0$ such that $B\left(z, \rho_{z}\right) \cap\left(E^{c}\right)_{\infty}$ is non-empty. Thus we have both $\mu_{\infty}\left(B\left(z, \rho_{z}\right) \cap(E)_{\infty}\right)>0$ and $\mu_{\infty}\left(B\left(z, \rho_{z}\right) \cap\left(E^{c}\right)_{\infty}\right)>0$. Since $\mu_{\infty}\left(X_{\infty} \backslash\left[(E)_{\infty} \cup\left(E^{c}\right)_{\infty}\right]\right)=0$, it follows from the relative isoperimetric inequality (2.6) applied to the ball $B\left(z, \rho_{z}\right)$, that we must have $P\left((E)_{\infty}, U\right) \geq P\left((E)_{\infty}, B\left(z, \rho_{z}\right)\right)>0$, contradicting the fact that $P\left((E)_{\infty}, U\right)=0$. Thus $(E)_{\infty}=U$ and similarly $\left(E^{c}\right)_{\infty}=V$.

Remark 5.6. If $\mu$ is an Ahlfors $s$-regular measure for some $s>1$ (recall (2.9)), then it is straightforward to verify that $\mu_{\infty}$ is also Ahlfors $s$-regular in $X_{\infty}$, and then by Theorems 5.4 and 5.5, $P\left((E)_{\infty}, \cdot\right)$ (and $\left.\pi_{\infty}\right)$ are Ahlfors $(s-1)$-regular measures in $X_{\infty}$. This corresponds to what we get in a Euclidean space $\mathbb{R}^{n}$, for $s=n$.

\section{ASYMPtotiC QUASI-LEAST GRADIENT PROPERTY}

From Theorem 4.9 we now know that asymptotic limits ( $\mu$-a.e.) of a BV function outside of the Cantor and jump parts of the function are of least gradient. We will show in this section that at co-dimension 1 almost every point of the measure-theoretic boundary of a set $E$ of finite perimeter, any limit set $(E)_{\infty}$ is a set of quasiminimal boundary surface as defined in [25], that is, $\chi_{(E)_{\infty}}$ is of quasi-least gradient. First we develop some preliminary results that are also of independent interest. 
6.1. Asymptotic minimality for sets of finite perimeter. The following theorem shows that given a set $E$ of finite perimeter, at essentially almost every point in $\partial^{*} E$ the set $E$ is asymptotically a minimal surface; compare this to [1, Proposition 5.7], where a weaker notion of asymptotic quasiminimality is established, where the quasiminimality condition requires to compare (locally) the perimeter of $E$ with the perimeter of modifications of $E$ by balls alone.

Theorem 6.1. Let $E \subset X$ be a set of finite perimeter. Then

$$
\lim _{r \rightarrow 0}\left(\frac{\inf _{u \in B V_{c}\left(B\left(x_{0}, r\right)\right)} V\left(\chi_{E}+u, B\left(x_{0}, r\right)\right)}{P\left(E, B\left(x_{0}, r\right)\right)}\right)=1
$$

for $P(E, \cdot)$-a.e. $x \in X$.

Proof. Note that the zero function is in $B V_{c}\left(B\left(x_{0}, r\right)\right)$, and so for each $r>0$,

$$
\frac{\inf _{u \in B V_{c}\left(B\left(x_{0}, r\right)\right)} V\left(\chi_{E}+u, B\left(x_{0}, r\right)\right)}{P\left(E, B\left(x_{0}, r\right)\right)} \leq 1 .
$$

Hence to prove the theorem, it suffices to show that

$$
\liminf _{r \rightarrow 0}\left(\frac{\inf _{u \in B V_{c}\left(B\left(x_{0}, r\right)\right)} V\left(\chi_{E}+u, B\left(x_{0}, r\right)\right)}{P\left(E, B\left(x_{0}, r\right)\right)}\right) \geq 1
$$

Let

$$
A:=\left\{x \in X: \liminf _{r \rightarrow 0}\left(\frac{\inf _{u \in B V_{c}(B(x, r))} V\left(\chi_{E}+u, B(x, r)\right)}{P(E, B(x, r))}\right)<1\right\}
$$

Note that $A$ is the increasing limit of sets $A_{n}$ where

$$
A_{n}:=\left\{x \in X: \liminf _{r \rightarrow 0}\left(\frac{\inf _{u \in B V_{c}(B(x, r))} V\left(\chi_{E}+u, B(x, r)\right)}{P(E, B(x, r))}\right)<1-\frac{1}{n}\right\}, \quad n \in \mathbb{N} .
$$

It therefore suffices to show that each $A_{n}$ satisfies $P\left(E, A_{n}\right)=0$. To this end, fix $n \in \mathbb{N}$. Then for every $x \in A_{n}$, there exist $r_{i}^{x} \rightarrow 0$ and $u_{i}^{x} \in B V_{c}\left(B\left(x, r_{i}^{x}\right)\right)$ with

$$
\frac{V\left(\chi_{E}+u_{i}^{x}, B\left(x, r_{i}^{x}\right)\right)}{P\left(E, B\left(x, r_{i}^{x}\right)\right)}<1-n^{-1}
$$

Furthermore, as $P(E, X)<\infty$, for every $x \in X$ we have $P(E, \partial B(x, r))=0$ for $\mathcal{H}^{1}$-almost every $r>0$. We can therefore choose $r_{i}^{x}>0$ such that in addition to the above, $P\left(E, \partial B\left(x, r_{i}^{x}\right)\right)=0$ for every $x \in A_{n}$. This is because as $u_{i}^{x}$ has compact support in $B\left(x, r_{i}^{x}\right)$, we can choose a smaller $r_{i}^{x}$ such that the support of $u_{i}^{x}$ is still contained in this smaller ball. Fix $k \in \mathbb{N}$ such that $1 / k<\frac{1}{4} \operatorname{diam} X$. The collection $\left\{\bar{B}\left(x, r_{i}^{x}\right): 0<r_{i}^{x}<1 / k\right\}_{x \in A_{n}}$ is a fine cover of $A_{n}$, that is, for every $x \in A_{n}$ we have $\inf _{i} r_{i}^{x}=0$. By $(2.13)$ we know that $P(E, \cdot)$ is asymptotically doubling, and so it satisfies the Vitali covering theorem, see [21, Theorem 3.4.3]. So we can pick a countable pairwise disjoint collection $\left\{B_{j}^{k}=B\left(x_{j}^{k}, r_{j}^{k}\right)\right\}_{j=1}^{\infty}=: \mathcal{G}_{k}$ such that, recalling also that $P(E, \partial B)=0$ for each $B \in \mathcal{G}_{k}$,

$$
P\left(E, A_{n} \backslash \bigcup_{B \in \mathcal{G}_{k}} B\right)=P\left(E, A_{n} \backslash \bigcup_{B \in \mathcal{G}_{k}} \bar{B}\right)=0 .
$$

We use the collection of balls $B_{j}^{k}$ to perturb the function $\chi_{E}$. Recall that for each ball $B_{j}^{k}$ there is a function $u_{j}^{k} \in B V_{c}\left(B_{j}^{k}\right)$ as in (6.1). Set

$$
h_{k}:=\chi_{E}+\sum_{j=1}^{\infty} u_{j}^{k}
$$


By the 1-Poincaré inequality (2.8) for compactly supported functions, for all $j \in \mathbb{N}$

$$
\int_{B_{j}^{k}}\left|u_{j}^{k}\right| d \mu \leq C r_{j}^{k} V\left(u_{j}^{k}, B_{j}^{k}\right) \leq C r_{j}^{k}\left[V\left(\chi_{E}+u_{j}^{k}, B_{j}^{k}\right)+V\left(\chi_{E}, B_{j}^{k}\right)\right] \leq C \frac{2-n^{-1}}{k} V\left(\chi_{E}, B_{j}^{k}\right) .
$$

Therefore by the pairwise disjointness of the balls in the collection $\mathcal{G}_{k}$,

$$
\int_{X}\left|\chi_{E}-h_{k}\right| d \mu \leq \sum_{j=1}^{\infty} \int_{B_{j}^{k}}\left|u_{j}^{k}\right| d \mu \leq \frac{C}{k} V\left(\chi_{E}, X\right) .
$$

Therefore $h_{k} \rightarrow \chi_{E}$ in $L^{1}(X)$ as $k \rightarrow \infty$. By the lower semicontinuity of the total variation,

$$
V\left(\chi_{E}, X\right) \leq \liminf _{k \rightarrow \infty} V\left(h_{k}, X\right) .
$$

For ease of notation, for each $j \in \mathbb{N}$ let

$$
G_{k, j}:=\bigcup_{i=1}^{j} \bar{B}_{i}^{k} \quad \text { and } \quad h_{k, j}:=u+\sum_{i=1}^{j} u_{i}^{k} .
$$

Now

$$
\begin{aligned}
V\left(h_{k, j}, G_{k, j}\right) & \leq V\left(h_{k, j}, \bigcup_{i=1}^{j} B_{i}^{k}\right)+V\left(h_{k, j}, \bigcup_{i=1}^{j} \partial B_{i}^{k}\right) \\
& =V\left(h_{k, j}, \bigcup_{i=1}^{j} B_{i}^{k}\right)+\sum_{i=1}^{j} V\left(\chi_{E}, \partial B_{i}^{k}\right) \\
& =V\left(h_{k, j}, \bigcup_{i=1}^{j} B_{i}^{k}\right)
\end{aligned}
$$

and so $V\left(h_{k, j}, G_{k, j}\right)=V\left(h_{k, j}, \bigcup_{i=1}^{j} B_{i}^{k}\right)$. Since $G_{k, j}$ is a closed set, it follows that

$$
\begin{aligned}
V\left(h_{k, j}, X\right) & =V\left(h_{k, j}, G_{k, j}\right)+V\left(h_{k, j}, X \backslash G_{k, j}\right) \\
& =\sum_{i=1}^{j} V\left(h_{k, j}, B_{i}^{k}\right)+V\left(\chi_{E}, X \backslash G_{k, j}\right) \\
& <\left(1-n^{-1}\right) \sum_{i=1}^{j} V\left(\chi_{E}, B_{i}^{k}\right)+V\left(\chi_{E}, X \backslash G_{k, j}\right) \quad \text { by }(6.1) \\
& =V\left(\chi_{E}, X\right)-n^{-1} V\left(\chi_{E}, \bigcup_{i=1}^{j} B_{i}^{k}\right)
\end{aligned}
$$

Therefore

$$
\begin{aligned}
V\left(h_{k}, X\right) \leq \liminf _{j \rightarrow \infty} V\left(h_{k, j}, X\right) & \leq V\left(\chi_{E}, X\right)-n^{-1} \lim _{j \rightarrow \infty} V\left(\chi_{E}, \bigcup_{i=1}^{j} B_{i}^{k}\right) \\
& =V\left(\chi_{E}, X\right)-n^{-1} V\left(\chi_{E}, \bigcup_{B \in \mathcal{G}_{k}} B\right) .
\end{aligned}
$$


Set $K_{k}:=\bigcup_{B \in \mathcal{G}_{k}} B$ and $F_{k}:=A_{n} \backslash K_{k}$ for each $k \in \mathbb{N}$. We have $P\left(E, F_{k}\right)=0$ for each $k \in \mathbb{N}$ by (6.2). For $F:=\bigcup_{k=1}^{\infty} F_{k}$ and $K:=\bigcap_{k=1}^{\infty} K_{k}$ we then have $P(E, F)=0$. In light of (6.3) and (6.4), we have

$$
P(E, X) \leq P(E, X)-\liminf _{k \rightarrow \infty} n^{-1} P\left(E, K_{k}\right) \leq P(E, X)-n^{-1} P(E, K),
$$

and so $P(E, K)=0$. Since $A_{n} \subset F \cup K$, it follows that $P\left(E, A_{n}\right)=0$. This completes the proof.

A similar analysis can be carried out for functions $u \in B V(X)$ with slightly more involved computations to obtain analogous asymptotic minimality results for $u$; we do not do so here as we have a stronger result for $u$ outside its jump and Cantor sets in Theorem 4.9. In Theorem 4.9 we obtain the global least gradient property of $u_{\infty}$ on the tangent space $X_{\infty}$, whereas in Theorem 6.1 above we do not consider least gradient properties of an asymptotic (blow-up) limit of $E$ in the tangent space $X_{\infty}$, but consider the asymptotic least gradient property of $\chi_{E}$ in $X$ itself. In the next subsection, we will explore analogous properties of the blow-up limit $(E)_{\infty}$, but unlike in Theorem 6.1 we do not have $(E)_{\infty}$ to be of least gradient in $X_{\infty}$, but merely a quasi-least gradient, see Theorem 6.3. This turns out to be not merely an artifact of our proof, but a real obstacle as demonstrated by Example 6.13 below.

6.2. Quasiminimality at almost every point. In this subsection we finally prove the quasiminimality property of the asymptotic limit set $(E)_{\infty}$.

Definition 6.2. A set $E \subset X$ is said to be $K$-quasiminimal, $K \geq 1$, if for every $B(x, R) \subset X$ and every $\phi \in B V_{c}(B(x, R))$ we have

$$
\frac{1}{K} P(E, B(x, R)) \leq V\left(\chi_{E}+\phi, B(x, R)\right) .
$$

Without loss of generality and applying a truncation, one can restrict attention to $\phi$ with values in $[-1,1]$, and such that $\chi_{E}+\phi$ has values in $[0,1]$.

The asymptotic minimality of $E$ (Theorem 6.1 ) can be upgraded to quasiminimality at generic tangents of the limit set $(E)_{\infty}$. In terms of notation, here we only consider the sequence

$$
\left(X_{n}, d_{n}, x, \mu_{n}\right):=\left(X, \frac{1}{r_{n}} \cdot d, x, \frac{1}{\mu\left(B\left(x, r_{n}\right)\right)} \cdot \mu\right)
$$

under the pointed measured Gromov-Hausdorff convergence, with $r_{n} \searrow 0$, see the discussion in Section 3.

Theorem 6.3. Let $E \subset X$ be a set of finite perimeter. Then, for $P(E, \cdot)$-almost every $x \in X$ and for any space $\left(X_{\infty}, d_{\infty}, x_{\infty}, \mu_{\infty}\right)$ arising as a pointed measured Gromov-Hausdorff tangent at $x$, any set $(E)_{\infty}$ that arises as an asymptotic limit of $E$ along some sequence $r_{n} \searrow 0$ is a Kquasiminimizer. Here $K$ depends only on the constants $C_{d}, C_{P}$.

The proof involves lifting Lipschitz functions with small energy to the sequence, and a pasting argument. The desired quasiminimality estimate then follows using Theorem 6.1 for the lifted sequence. We need the following general BV approximation theorem, which is an analog of [11, Lemma 5.2] for $p=1$. We follow the arguments of Cheeger.

Proposition 6.4. Let $f \in B V(X)$. Then, there exist Lipschitz continuous $f_{i}$ with bounded Lipschitz continuous upper gradients $v_{i}$ such that $f_{i} \rightarrow f$ in $L_{l o c}^{1}(X)$ and $v_{i} d \mu \stackrel{*}{\rightarrow} d V(f, \cdot)$. 
To prove this proposition we define the following auxiliary function. For a nonnegative Borel function $g$ on $X$ we set $\mathcal{F}_{g}: X \times X \rightarrow[0, \infty]$ to be

$$
\mathcal{F}_{g}^{X}\left(x_{1}, x_{2}\right):=\mathcal{F}_{g}\left(x_{1}, x_{2}\right):=\inf _{\gamma} \int_{\gamma} g d s
$$

whenever $x_{1}, x_{2} \in X$. If $x_{1}=x_{2}$, we set $\mathcal{F}_{g}\left(x_{1}, x_{2}\right)=0$. The infimum is taken over all rectifiable curves $\gamma$ connecting $x_{1}$ to $x_{2}$. Note that by the definition of upper gradient (2.3), we have that if $g$ is an upper gradient of a function $f: X \rightarrow \mathbb{R}$, then for every $x, y \in X$,

$$
|f(x)-f(y)| \leq \mathcal{F}_{g}(x, y) \text {. }
$$

For the proof of the following lemma see [11, Lemma 5.18] or [20, pp. 13-14].

Lemma 6.5. Fix $\eta>0$. Let $g: X \rightarrow[\eta, \infty)$ be a countably valued lower semicontinuous function. Then for $g_{n} \geq \eta$ an increasing sequence of Lipschitz continuous functions on $X$ converging pointwise $g_{n} \nearrow g$, we have that for every $x, y \in X$,

$$
\mathcal{F}_{g}(x, y)=\lim _{n \rightarrow \infty} \mathcal{F}_{g_{n}}(x, y) .
$$

Moreover, such a sequence $g_{n}$ exists.

Lemma 6.6. Let $f \in B V(X)$. Then there is a sequence of Lipschitz functions $f_{k}$ on $X$ such that $f_{k} \rightarrow f$ in $L_{l o c}^{1}(X)$ and $\operatorname{lip} f_{k} d \mu \stackrel{*}{\rightarrow} d V(f, \cdot)$.

Proof. By the definition of the total variation we can find a sequence of locally Lipschitz functions $f_{k}$ and upper gradients $g_{k}=\operatorname{lip} f_{k}$ such that $f_{k} \rightarrow f$ in $L_{\mathrm{loc}}^{1}(X)$ and $\lim _{k} \int_{X} g_{k} d \mu=V(f, X)$. Multiplying with suitable cutoff functions if necessary, we can assume that the $f_{k}$ are Lipschitz. For any open set $U \subset X$, we have by the definition of the total variation that

$$
V(f, U) \leq \liminf _{k \rightarrow \infty} \int_{U} g_{k} d \mu .
$$

On the other hand, for any closed set $F \subset X$ we have

$$
V(f, X)=\lim _{k \rightarrow \infty} \int_{X} g_{k} d \mu \geq \limsup _{k \rightarrow \infty} \int_{F} g_{k} d \mu+\liminf _{k \rightarrow \infty} \int_{X \backslash F} g_{k} d \mu \geq \limsup _{k \rightarrow \infty} \int_{F} g_{k} d \mu+V(f, X \backslash F),
$$

where the last inequality again follows by the definition of the total variation. Thus

$$
\limsup _{k \rightarrow \infty} \int_{F} g_{k} d \mu \leq V(f, F)
$$

According to a standard characterization of the weak* convergence of Radon measures, see e.g. [12, p. 54], the above inequality and (6.5) together give $g_{k} d \mu \stackrel{*}{\rightarrow} d V(f, \cdot)$.

Lemma 6.7. Let $f$ be a nonnegative Lipschitz function on $X$ and $g \in L_{l o c}^{1}(X)$ a bounded countably valued lower semicontinuous upper gradient of $f$. Suppose that there is a $\tau>0$ such that $g \geq \tau$ on $X$. Then there is a sequence $f_{k}$ of Lipschitz continuous functions on $X$ with $f_{k} \rightarrow f$ in $L_{l o c}^{1}(X)$ and bounded Lipschitz continuous upper gradients $g_{k}$ of $f_{k}$ such that $g_{k} \rightarrow g$ in $L_{l o c}^{1}(X)$ and $g_{k}$ monotone increases to $g$ everywhere on $X$, and $g_{k} \geq \tau$ for each $k$.

Proof. Since $g$ is lower semicontinuous, we can find a sequence of Lipschitz continuous functions $g_{k} \geq \tau$ on $X$ such that $g_{k} \rightarrow g$ in $L_{\text {loc }}^{1}(X)$ and in addition $g_{k} \leq g_{k+1} \leq g$ on $X$ for each $k \in \mathbb{N}$. By Lemma 6.5 we know that $\mathcal{F}_{g}=\lim _{k} \mathcal{F}_{g_{k}}$ pointwise everywhere on $X \times X$. 
Next, we fix $x_{0} \in X$ and for each positive integer $i$ let $\widehat{A_{i}}$ be a maximal $1 / i$-net of $X$ such that $\widehat{A_{i}} \subset \widehat{A_{i+1}}$ for each $i \in \mathbb{N}$, and let $A_{i}=\widehat{A_{i}} \cap B\left(x_{0}, 2 i\right)$. Then $A_{i} \subset A_{i+1}$, and by the doubling property of $\mu$ we know that $A_{i}$ is a finite set for each $i$. As $g$ is bounded, we can also ensure that each $g_{k} \leq M$ and $g \leq M$ on $X$ for some positive $M$. Therefore for each $y \in X$ we know that $\mathcal{F}_{g}$ and $\mathcal{F}_{g_{k}}$ are $M C$-Lipschitz where $C$ is the quasiconvexity constant of $X$. Now, taking inspiration from the McShane extension (see also [11]), we set

$$
f_{k}(x):=\inf \left\{f(y)+\mathcal{F}_{g_{k}}(x, y): y \in A_{k}\right\} .
$$

Then $f_{k}$ is also $M C$-Lipschitz on $X$. A standard argument (see e.g. [21, p. 384]) shows that $g_{k}$ is an upper gradient of $f_{k}$.

For $x \in \bigcup_{n} A_{n}$, we choose $n \in \mathbb{N}$ such that $x \in A_{n}$; then for $k \geq n+1$ we see that $x \in A_{k}$. It follows that $f_{k}(x) \leq f(x)$. If $y \in X \backslash B(x, L)$ for some $L>0$ then as $f$ is nonnegative, $f(y)+\mathcal{F}_{g_{k}}(x, y) \geq L \tau$; thus to obtain $f_{k}(x)$ it suffices to look only at $y \in A_{k} \cap B(x, L)$ where $L=[1+f(x)] / \tau$. Let $y_{k} \in A_{k} \cap B(x, L)$ such that

$$
k^{-1}+f_{k}(x) \geq f\left(y_{k}\right)+\mathcal{F}_{g_{k}}\left(x, y_{k}\right) .
$$

Then the sequence $\left(y_{k}\right)$ lies in the compact set $\bar{B}(x, L)$ and hence has a subsequence $y_{k_{j}}$ converging to some $y_{\infty} \in \bar{B}(x, L)$. Thus $f(x) \geq \lim _{k \rightarrow \infty} f_{k}(x) \geq f\left(y_{\infty}\right)+\lim _{k \rightarrow \infty} \mathcal{F}_{g_{k}}\left(x, y_{k}\right)$. Observe that

$$
\left|\mathcal{F}_{g_{k}}\left(x, y_{k}\right)-\mathcal{F}_{g_{k}}\left(x, y_{\infty}\right)\right| \leq M C d\left(y_{k}, y_{\infty}\right) .
$$

It then follows from Lemma 6.5 that

$$
f(x) \geq \lim _{k \rightarrow \infty} f_{k}(x) \geq f\left(y_{\infty}\right)+\lim _{k \rightarrow \infty} \mathcal{F}_{g_{k}}\left(x, y_{\infty}\right)=f\left(y_{\infty}\right)+\mathcal{F}_{g}\left(x, y_{\infty}\right) \geq f(x),
$$

and it then follows that $\lim _{k \rightarrow \infty} f_{k}(x)=f(x)$. Now the uniform Lipschitz continuity of $f_{k}, k \in \mathbb{N}$ and $f$ shows that $\lim _{k} f_{k}=f$ pointwise on $X$. An appeal to the Lebesgue dominated convergence theorem (and the fact that $f_{k} \leq\|f\|_{L^{\infty}(B)}+M k<\infty$ on the ball $B=B\left(x_{0}, k\right)$ ) yields the convergence also in $L_{\text {loc }}^{1}(X)$.

The above lemmas allow us now to prove Proposition 6.4.

Proof of Proposition 6.4. By Lemma 6.6 we obtain a sequence $f_{k}$ of Lipschitz functions on $X$ with $f_{k} \rightarrow f$ in $L_{\text {loc }}^{1}(X)$ and upper gradients $g_{k}=\operatorname{lip} f_{k}$ of $f_{k}$ such that $g_{k} d \mu \stackrel{*}{\rightarrow} d V(f, \cdot)$. Note that each $g_{k}$ is bounded. By the Vitali-Carathéodory theorem, see e.g. [21, p. 108], for each $k$ we can find a bounded countably valued lower semicontinuous function $g_{k}^{\prime} \geq g_{k}$ such that $\left\|g_{k}^{\prime}-g_{k}\right\|_{L^{1}(X)} \rightarrow 0$ as $k \rightarrow \infty$. Note that automatically $g_{k}^{\prime}$ is also an upper gradient of $f_{k}$. Moreover, we now have $g_{k}^{\prime} d \mu \stackrel{*}{\rightarrow} d V(f, \cdot)$, and so we also have $\left[g_{k}^{\prime}+k^{-1}\right] d \mu \stackrel{*}{\rightarrow} d V(f, \cdot)$.

Next we apply Lemma 6.7 to obtain bounded Lipschitz functions $v_{k}$ and Lipschitz functions $F_{k}$ such that $v_{k}$ is an upper gradient of $F_{k}, F_{k} \rightarrow f$ in $L_{\text {loc }}^{1}(X)$, and $v_{k}-\left[g_{k}^{\prime}+k^{-1}\right] \rightarrow 0$ in $L_{\text {loc }}^{1}(X)$ as $k \rightarrow \infty$. It follows then also that $v_{k} d \mu \stackrel{*}{\rightarrow} d V(f, \cdot)$, completing the proof of the proposition.

We will need the following lemma from Keith [24, Proposition 4], see also [20, proof of Proposition 2.17]. This lemma is a simple consequence of the Arzelà-Ascoli theorem together with the lower semicontinuity of $g$. In the lemmas below we will consider curves $\gamma_{n}$ to be arc length parametrized in the sense that each $\gamma_{n}:[0, L] \rightarrow Z$ such that $\left.\gamma_{n}\right|_{\left[0, \ell\left(\gamma_{n}\right)\right]}$ is arc-length parametrized, and $\gamma_{n}$ is constant on $\left[\ell\left(\gamma_{n}\right), L\right]$. Here, $\ell\left(\gamma_{n}\right)$ denotes the length of $\gamma_{n}$. Of course, the limit curve $\gamma_{\infty}$ may not be arc-length parametrized in the above sense, but is sub-arc-length parametrized in the sense that $\ell\left(\left.\gamma\right|_{\left[t_{1}, t_{2}\right]}\right) \leq t_{2}-t_{1}$ for any $0 \leq t_{1}<t_{2} \leq L$. 
Lemma 6.8. [24, Proposition 4] Let $Z$ be a proper metric space and $g: Z \rightarrow \mathbb{R}$ a nonnegative lower semicontinuous function. If $L>0, K \subset Z$ a compact set, and $\left(\gamma_{n}\right)_{n}$ is a sequence of curves in $Z$ with length at most $L$ such that each $\gamma_{n}$ is contained in $K$, then there exists a rectifiable curve $\gamma_{\infty}$ so that a subsequence of $\gamma_{n}$ converges to $\gamma_{\infty}$ uniformly. For such $\gamma_{\infty}$ we also have that

$$
\int_{\gamma_{\infty}} g d s \leq \liminf _{n \rightarrow \infty} \int_{\gamma_{n}} g d s .
$$

As a corollary, we obtain the following.

Lemma 6.9. Let $g: Z \rightarrow[\tau, \infty)$ be a nonnegative lower semicontinuous function on a proper space $Z$ for some $\tau>0$, and assume that $x_{n} \rightarrow x$ and $y_{n} \rightarrow y$ are sequences of points in $Z$. Then,

$$
\mathcal{F}_{g}(x, y) \leq \liminf _{n \rightarrow \infty} \mathcal{F}_{g}\left(x_{n}, y_{n}\right) .
$$

Note that we avoid assuming $Z$ has any rectifiable curves, or that it is quasiconvex. This is necessary for our application where $Z$ is the proper metric space into which the sequence of scaled spaces $X_{i}$ and the tangent space $X_{\infty}$ embed isometrically as described in the latter part of Remark 3.2.

Proof. If the limit infimum on the right hand side of (6.6) is infinite, there is nothing to prove. So we will assume that it is finite. By passing to a subsequence, we can assume that there is some real number $M>0$ such that $\mathcal{F}_{g}\left(x_{n}, y_{n}\right) \leq M$ for all $n$. Then for every $0<\epsilon<M$, there exist curves $\gamma_{n}$ connecting $x_{n}$ and $y_{n}$ such that

$$
\tau \ell\left(\gamma_{n}\right) \leq \int_{\gamma_{n}} g d s \leq \mathcal{F}_{g}\left(x_{n}, y_{n}\right)+\epsilon \leq 2 M
$$

Since $\gamma_{n}$ connects $x_{n}$ to $y_{n}$, and these converge, respectively, to $x$ and $y$, the curves $\gamma_{n}$ lie, for sufficiently large $n$, in the closed ball $\overline{B(x, M+2 M / \tau)}$ which is compact. Then, by Lemma 6.8 , by taking a subsequence if necessary, the sequence $\gamma_{n}$ converges to some curve $\gamma_{\infty}$, and

$$
\mathcal{F}_{g}(x, y) \leq \int_{\gamma_{\infty}} g d s \leq \liminf _{n \rightarrow \infty} \int_{\gamma_{n}} g d s \leq \liminf _{n \rightarrow \infty} \mathcal{F}_{g}\left(x_{n}, y_{n}\right)+\epsilon .
$$

Since this holds for every small $\epsilon>0$ the claim follows.

Lemma 6.10. Let $\left(X_{i}, d_{i}, x_{i}, \mu_{i}\right) \rightarrow\left(X_{\infty}, d_{\infty}, x_{\infty}, \mu_{\infty}\right)$ be a sequence of scaled (from $\left.X\right)$ metric measure spaces converging in the pointed measured Gromov-Hausdorff sense. If $f$ is a nonnegative Lipschitz function on $X_{\infty}$, with a bounded Lipschitz upper gradient $v$, then there exists a subsequence, also denoted $\left(X_{i}, d_{i}, x_{i}, \mu_{i}\right)$, and uniformly Lipschitz continuous functions $f_{i}$ with Lipschitz continuous upper gradients $v_{i}$ on $X_{i}$ such that

$$
v_{i} d \mu_{i} \stackrel{*}{\rightarrow} v d \mu_{\infty}
$$

and $f$ is a limit function of $f_{i}$ in the sense of (3.3).

Proof. Without loss of generality, we can assume that $v \geq \tau$ for some positive $\tau$, since otherwise we can obtain the result by considering $\max \{v, 1 / k\}$ instead of $v$ for each positive integer $k$, and then complete the proof with the help of a diagonalization argument, letting $k \rightarrow \infty$.

Let $\hat{v}: Z \rightarrow \mathbb{R}$ be a McShane extension of the Lipschitz function $\left.v \circ \iota\right|_{\iota\left(X_{\infty}\right)} ^{-1}$ on $\iota\left(X_{\infty}\right)$ to the entirety of $Z$. Also, such an extension can be chosen to be bounded and so that $\hat{v} \geq \tau$. Let $v_{i}:=\hat{v} \circ \iota_{i}: X_{i} \rightarrow \mathbb{R}$. 
Next, let $\hat{f}: Z \rightarrow \mathbb{R}$ be constructed similarly, by first setting $\hat{f}(z):=f \circ \iota^{-1}(z)$ for $z \in \iota\left(X_{\infty}\right)$, and then taking a McShane extension to $Z$. We can choose $\hat{f}$ to be nonnegative. Next we construct the functions $f_{i}: X_{i} \rightarrow \mathbb{R}$ so that $v_{i}$ is an upper gradient of $f_{i}$ as follows. For $x \in X_{i}$ we set

$$
f_{i}(x):=\inf _{y \in X_{i}}\left[\hat{f}\left(\iota_{i}(y)\right)+\mathcal{F}_{\hat{v}}^{\iota_{i}\left(X_{i}\right)}\left(\iota_{i}(y), \iota_{i}(x)\right)\right]=\inf _{y \in X_{i}}\left[\hat{f}\left(\iota_{i}(y)\right)+\mathcal{F}_{v_{i}}^{X_{i}}(y, x)\right] .
$$

For ease of notation, we set $\mathcal{F}_{v_{i}}(x, y):=\mathcal{F}_{\hat{v}}^{\iota_{i}\left(X_{i}\right)}\left(\iota_{i}(y), \iota_{i}(x)\right)$ for $x, y \in X_{i}$. From the definition of $f_{i}$ it is clear that $f_{i}(x) \leq \hat{f}\left(\iota_{i}(x)\right)$ for each $x \in X_{i}$. Also, $f_{i}$ is nonnegative, and has $v_{i}$ as an upper gradient.

We will now show that $f$ is a limit function of $f_{i}$. To do so, we need to show for every $r>0$,

$$
\lim _{i \rightarrow \infty}\left\|f-f_{i} \circ \phi_{i}\right\|_{L^{\infty}\left(B_{X_{\infty}}\left(x_{\infty}, r\right)\right)}=0,
$$

where $\phi_{i}$ are the approximating maps from Definition 3.1. Suppose this is not the case. Then there is some $r>0$ and some $\delta>0$ such that, by passing to a subsequence if needed, we have

$$
\liminf _{i \rightarrow \infty}\left\|f-f_{i} \circ \phi_{i}\right\|_{L^{\infty}\left(B_{X_{\infty}}\left(x_{\infty}, r\right)\right)}>\delta .
$$

Thus, for each $i$ there is a point $x_{i} \in B_{X_{\infty}}\left(x_{\infty}, r\right)$ such that

$$
\left|f\left(x_{i}\right)-f_{i}\left(\phi_{i}\left(x_{i}\right)\right)\right|>\delta \text {. }
$$

Since $X_{\infty}$ is proper and $x_{i} \in B_{X_{\infty}}\left(x_{\infty}, r\right)$ for all $i$, there is a subsequence, also denoted with the index $i$, such that $x_{i} \rightarrow x \in X_{\infty}$. Fix $\delta>0$. Then from the definition of $f_{i}\left(\phi_{i}\left(x_{i}\right)\right)$, we have $y_{i} \in X_{i}$ such that

$$
\left|f_{i}\left(\phi_{i}\left(x_{i}\right)\right)-\hat{f}\left(\iota_{i}\left(y_{i}\right)\right)-\mathcal{F}_{v_{i}}\left(y_{i}, \phi_{i}\left(x_{i}\right)\right)\right| \leq \delta / 4 \text {. }
$$

Combining the above with (6.8) we get

$$
\left|f\left(x_{i}\right)-\hat{f}\left(\iota_{i}\left(y_{i}\right)\right)-\mathcal{F}_{v_{i}}\left(y_{i}, \phi_{i}\left(x_{i}\right)\right)\right| \geq \delta / 2 .
$$

By (6.9) we have $\hat{f}\left(\iota_{i}\left(y_{i}\right)\right)+\mathcal{F}_{v_{i}}\left(y_{i}, \phi_{i}\left(x_{i}\right)\right) \leq f_{i}\left(\phi_{i}\left(x_{i}\right)\right)+\delta / 4 \leq \hat{f}\left(\iota_{i}\left(\phi_{i}\left(x_{i}\right)\right)\right)+\delta / 4$, and so the triangle inequality gives

$$
\left|f\left(x_{i}\right)-\hat{f}\left(\iota_{i}\left(\phi_{i}\left(x_{i}\right)\right)\right)\right|+\hat{f}\left(\iota_{i}\left(\phi_{i}\left(x_{i}\right)\right)\right)-\hat{f}\left(\iota_{i}\left(y_{i}\right)\right)-\mathcal{F}_{v_{i}}\left(y_{i}, \phi_{i}\left(x_{i}\right)\right) \geq \delta / 4 .
$$

For the first term, note that $f\left(x_{i}\right)=\hat{f}\left(\iota\left(x_{i}\right)\right)$, and from (3.1) we get $\lim _{i \rightarrow \infty} d_{Z}\left(\iota\left(x_{i}\right), \iota_{i}\left(\phi_{i}\left(x_{i}\right)\right)\right)=0$, and thus from the Lipschitz continuity of $\hat{f}$,

$$
\lim _{i \rightarrow \infty}\left|\hat{f}\left(\iota\left(x_{i}\right)\right)-\hat{f}\left(\iota_{i}\left(\phi_{i}\left(x_{i}\right)\right)\right)\right|=0 .
$$

Since $\lim _{i} x_{i}=x$, we also have

$$
\begin{aligned}
d_{Z}\left(\iota_{i}\left(\phi_{i}\left(x_{i}\right)\right), \iota(x)\right) & \leq d_{Z}\left(\iota_{i}\left(\phi_{i}\left(x_{i}\right)\right), \iota\left(x_{i}\right)\right)+d_{Z}\left(\iota\left(x_{i}\right), \iota(x)\right) \\
& =d_{Z}\left(\iota_{i}\left(\phi_{i}\left(x_{i}\right)\right), \iota\left(x_{i}\right)\right)+d_{X_{\infty}}\left(x_{i}, x\right) \rightarrow 0 \quad \text { as } i \rightarrow \infty .
\end{aligned}
$$

Therefore the sequence of real numbers $\hat{f}\left(\iota_{i}\left(\phi_{i}\left(x_{i}\right)\right)\right)$ is bounded, that is, there is some $M>\delta>$ 0 such that $\sup _{i} \hat{f}\left(\iota_{i}\left(\phi_{i}\left(x_{i}\right)\right)\right) \leq M$. The functions $v_{i}$ are bounded from below by $\tau$ and $f$ is nonnegative. Therefore, if $d\left(\phi_{i}\left(x_{i}\right), y_{i}\right)>2 M / \tau$, then

$$
\hat{f}\left(\iota_{i}\left(y_{i}\right)\right)+\mathcal{F}_{v_{i}}\left(y_{i}, \phi_{i}\left(x_{i}\right)\right) \geq \mathcal{F}_{v_{i}}^{X_{i}}\left(y_{i}, \phi_{i}\left(x_{i}\right)\right) \geq 2 M>\hat{f}\left(\iota_{i}\left(\phi_{i}\left(x_{i}\right)\right)\right)+\delta \geq f_{i}\left(\phi_{i}\left(x_{i}\right)\right)+\delta,
$$

which would violate the choice of $y_{i}$, (6.9). Hence we must have $d\left(\phi_{i}\left(x_{i}\right), y_{i}\right) \leq 2 M / \tau$. As the sequence $\iota_{i}\left(\phi_{i}\left(x_{i}\right)\right)$ lies in a ball, in $Z$, centered at $\iota(x)$ by $(6.12)$, we see then that the sequence $\iota_{i}\left(y_{i}\right)$ also lies in a ball centered at $\iota(x)$. Therefore, by the properness of $Z$, there is a subsequence, 
also denoted with the index $i$, and a point $\hat{y} \in Z$ such that $\lim _{i} \iota_{i}\left(y_{i}\right)=\hat{y}$. As $y_{i} \in X_{i}$ and $X_{i}$ converges to the metric space $X_{\infty}$, it follows that $\hat{y}=\iota(y)$ for some $y \in X_{\infty}$. Then by Lemma 6.9 we get $\mathcal{F}_{\hat{v}}^{Z}(\iota(x), \iota(y)) \leq \liminf _{i \rightarrow \infty} \mathcal{F}_{\hat{v}}^{Z}\left(\iota_{i}\left(\phi_{i}\left(x_{i}\right)\right), \iota_{i}\left(y_{i}\right)\right)$. Note that $\mathcal{F}_{v_{i}}\left(y_{i}, \phi_{i}\left(x_{i}\right)\right)=$ $\mathcal{F}_{\hat{v}}^{\iota_{i}\left(X_{i}\right)}\left(\iota_{i}\left(y_{i}\right), \iota_{i}\left(\phi_{i}\left(x_{i}\right)\right)\right)$, which is not the same as $\mathcal{F}_{\hat{v}}^{Z}\left(\iota_{i}\left(y_{i}\right), \iota_{i}\left(\phi_{i}\left(x_{i}\right)\right)\right)$. However, we have that $\mathcal{F}_{\hat{v}}^{Z}\left(\iota_{i}\left(y_{i}\right), \iota_{i}\left(\phi_{i}\left(x_{i}\right)\right)\right) \leq \mathcal{F}_{\hat{v}}^{\iota_{i}\left(X_{i}\right)}\left(\iota_{i}\left(y_{i}\right), \iota_{i}\left(\phi_{i}\left(x_{i}\right)\right)\right)$. Now by (6.10) and (6.11), we obtain

$$
\begin{aligned}
\frac{\delta}{4}+\mathcal{F}_{\hat{v}}^{Z}(\iota(x), \iota(y)) & \leq \frac{\delta}{4}+\liminf _{i} \mathcal{F}_{\hat{v}}^{Z}\left(\iota_{i}\left(y_{i}\right), \iota_{i}\left(\phi_{i}\left(x_{i}\right)\right)\right) \\
& \leq \lim _{i}\left[\hat{f}\left(\iota_{i}\left(\phi_{i}\left(x_{i}\right)\right)\right)-\hat{f}\left(\iota_{i}\left(y_{i}\right)\right)\right]=\hat{f}(\iota(x))-\hat{f}(\iota(y))=f(x)-f(y) .
\end{aligned}
$$

We now use the specific structure of $Z$; by [22], we can choose $Z$ to be the completion of pairwise disjoint union of $X_{i}, i \in \mathbb{N}$. With such a choice, it follows that if $\gamma$ is a non-constant rectifiable curve in $Z$, then either $\gamma$ lies entirely in $\iota_{i}\left(X_{i}\right)$ for some positive integer $i$, or else $\gamma$ lies entirely in $\iota\left(X_{\infty}\right)$. It follows that

$$
\mathcal{F}_{\hat{v}}^{Z}(\iota(x), \iota(y))=\mathcal{F}_{\hat{v}}^{\iota\left(X_{\infty}\right)}(\iota(x), \iota(y))=\mathcal{F}_{v}^{X}(x, y) .
$$

Hence from the above inequality we obtain

$$
\mathcal{F}_{v}^{X_{\infty}}(x, y)<\frac{\delta}{4}+\mathcal{F}_{v}^{X_{\infty}}(x, y) \leq f(x)-f(y) \leq|f(x)-f(y)|,
$$

which is not possible as $v$ is an upper gradient of $f$. Thus (6.7) is false, and so $f=\lim _{i} f_{i}$ as desired.

Finally, we show that $v_{i} d \mu_{i} \stackrel{*}{\rightarrow} v d \mu_{\infty}$ as follows. Pick a test function $\phi \in C_{c}(Z)$. Then also $\phi \hat{v} \in C_{c}(Z)$. Using this fact and the fact that $\iota_{i, *} \mu_{i} \stackrel{*}{\rightarrow} \iota_{*} \mu_{\infty}$, we get

$$
\lim _{i \rightarrow \infty} \int_{Z} \phi \iota_{i, *}\left(v_{i} d \mu_{i}\right)=\lim _{i \rightarrow \infty} \int_{Z} \phi \hat{v} d \iota_{i, *} \mu_{i}=\int_{Z} \phi \hat{v} d \iota_{*} \mu_{\infty}=\int_{Z} \phi \iota_{*}\left(v d \mu_{\infty}\right) .
$$

We also need the following lemma, which stitches two given BV functions along an annulus to yield a BV function whose BV energy is controllable.

Lemma 6.11. [33, Lemma 3.3] Let $f \in B V(X), x \in X, 0<a<b \leq R$, and $g \in B V(B(x, b))$. Then there exists a $2 /(b-a)$-Lipschitz function $\eta: X \rightarrow[0,1]$ with compact support in $B(x, b)$, and such that $\eta=1$ on $B(x, a)$ such that $h=\eta g+(1-\eta) f \in B V(X)$ with

$$
V(h, B(x, R)) \leq V(f, B(x, R) \backslash \overline{B(x, a)})+V(g, B(x, b))+\frac{2}{b-a} \int_{B(x, b) \backslash B(x, a)}|f-g| d \mu .
$$

Finally, we can conclude the proof of Theorem 6.3 .

Proof of Theorem 6.3. Let $x \in \Sigma_{\gamma}$ be a point where the conclusions of Theorem 6.1, Lemma 2.2 and Theorem 5.5 hold. We will show that the corresponding asymptotic set $(E)_{\infty}$ is $K$-quasiminimal for some $K$, which will be determined at the end of the proof. Since $P(E, \cdot)$-almost every $x \in X$ is such a point, this concludes the proof. Let $R>0, z \in X_{\infty}$, and $\varphi \in B V_{c}\left(B_{X_{\infty}}(z, R)\right)$. By slightly decreasing $R$ if necessary, we can assume that $P\left((E)_{\infty}, \partial B_{X_{\infty}}(z, R)\right)=0$.

From Theorem 6.1, there is some $r_{0}>0$ such that that for every $r_{0}>r>0$ there is some positive $\varepsilon_{r}$ such that $\lim _{r \rightarrow 0^{+}} \varepsilon_{r}=0$ and whenever $\psi \in B V_{c}\left(B_{X}(x, r)\right)$, we have

$$
P\left(E, B_{X}(x, r)\right) \leq\left(1+\varepsilon_{r}\right) V\left(\chi_{E}+\psi, B_{X}(x, r)\right) .
$$

By a standard truncation argument, we can assume without loss of generality that $0 \leq \chi_{(E)_{\infty}}+$ $\varphi \leq 1$. By Proposition 6.4 we can find a sequence of Lipschitz function-Lipschitz upper gradient 
pairs $f_{i}, v_{i}$ on $X_{\infty}$, with each $v_{i}$ bounded, such that $f_{i} \rightarrow \chi_{(E)_{\infty}}+\varphi$ in $L_{\text {loc }}^{1}\left(X_{\infty}\right)$ and $v_{i} d \mu_{\infty} \stackrel{*}{\rightarrow}$ $d V\left(\chi_{(E)_{\infty}}+\varphi, \cdot\right)$. Next, for each positive integer $i$ we apply Lemma 6.10 to obtain lifts $f_{i, n}, v_{i, n}$ to $X_{n}$ such that $v_{i, n} d \mu_{n} \stackrel{*}{\rightarrow} v_{i} d \mu_{\infty}$ and $f_{i, n} \rightarrow f_{i}$. Further, by truncating each $f_{i}$ and $f_{i, n}$, we can also assume $0 \leq f_{i}, f_{i, n} \leq 1$.

By passing to a subsequence of $\left(X_{n}, d_{n}, x, \mu_{n}\right)$ if necessary, with $\rho$ fixed and chosen in the interval $\left[2\left[R+d_{X_{\infty}}\left(z, x_{\infty}\right)\right], 3\left[R+d_{X_{\infty}}\left(z, x_{\infty}\right)\right]\right]$, we have $\rho r_{n}<r_{0}$ and that

$$
\sup _{y, w \in B_{X_{\infty}}\left(x_{\infty}, \rho\right)}\left|d_{n}\left(\phi_{n}(y), \phi_{n}(w)\right)-d_{X_{\infty}}(y, w)\right|<\frac{1}{n},
$$

and

$$
B_{n}(x, \rho) \subset \underset{y \in \phi_{n}\left(B_{X_{\infty}}\left(x_{\infty}, \rho+1 / n\right)\right)}{\bigcup_{n}(y, 1 / n) .}
$$

For each $n$ we set $x_{n}:=\phi_{n}(z)$. By choosing $\rho$ appropriately, we can also ensure

$$
\pi_{\infty}\left(\partial B_{X_{\infty}}\left(x_{\infty}, \rho\right)\right)=0 .
$$

Then by the above,

$$
\frac{d_{X}\left(x, x_{n}\right)}{r_{n}}=d_{n}\left(x, x_{n}\right) \leq d_{X_{\infty}}\left(x_{\infty}, z\right)+\frac{1}{n} .
$$

Fix $\tau \in(0,1)$. We now use Lemma 6.11 to stitch $f_{i, n}$ on $B_{n}\left(x_{n}, R\right)$ to $\chi_{E}$ on $B_{n}(x, \rho) \backslash B_{n}\left(x_{n},[1+\right.$ $\tau] R)$ using the Lipschitz function $\eta_{n}$ to obtain $h_{i, n}:=\eta_{n} f_{i, n}+\left(1-\eta_{n}\right) \chi_{E}$. Then, since $\rho r_{n}<r_{0}$, we know that

$$
P\left(E, B_{X}\left(x, \rho r_{n}\right)\right) \leq\left(1+\varepsilon_{\rho r_{n}}\right) V\left(h_{i, n}, B_{X}\left(x, \rho r_{n}\right)\right) .
$$

Note by Lemma 6.11 that

$$
\begin{aligned}
V\left(h_{i, n}, B_{n}(x, \rho)\right) \leq & P_{n}\left(E, B_{n}(x, \rho) \backslash B_{n}\left(x_{n}, R\right)\right)+V\left(f_{i, n}, B_{n}\left(x_{n},[1+\tau] R\right)\right) \\
& +\frac{2}{\tau R} \int_{B_{n}\left(x_{n},[1+\tau] R\right) \backslash B_{n}\left(x_{n}, R\right)}\left|f_{i, n}-\chi_{E}\right| d \mu_{n} .
\end{aligned}
$$

Note that $h_{i, n}-\chi_{E}$ has compact support on $B_{n}(x, \rho)$ for large enough $n$ since we can ensure $B_{n}\left(x_{n},[1+\tau] R\right) \subset B_{n}(x, \rho)$. Combining this with the (asymptotic) minimality of $\chi_{E}$ at $x$ as explained above, we obtain that

$$
\begin{aligned}
P_{n}\left(E, B_{n}(x, \rho)\right) \leq[1 & \left.+\varepsilon_{\rho r_{n}}\right]\left(P_{n}\left(E, B_{n}(x, \rho) \backslash B_{n}\left(x_{n}, R\right)\right)+\int_{B_{n}\left(x_{n},[1+\tau] R\right)} v_{i, n} d \mu_{n}\right. \\
& \left.+\frac{2}{\tau R} \int_{B_{n}\left(x_{n},[1+\tau] R\right) \backslash B_{n}\left(x_{n}, R\right)}\left|f_{i, n}-\chi_{E}\right| d \mu_{n}\right) .
\end{aligned}
$$

In the above, we have also used the fact that as $v_{i, n}$ is an upper gradient of $f_{i, n}$, we have $d V\left(f_{i, n}, \cdot\right) \leq$ $v_{i, n} d \mu_{n}$.

Recall that $\chi_{E}$ is either 0 or 1 on $X_{\infty}$, and $0 \leq f_{i, n}, f_{i} \leq 1$, and so we have

$$
\left|f_{i, n}-\chi_{E}\right|=\left(1-f_{i, n}\right) \chi_{E}+\left(1-\chi_{E}\right) f_{i, n}
$$

and

$$
\left|f_{i}-\chi_{(E)_{\infty}}\right|=\left(1-f_{i}\right) \chi_{(E)_{\infty}}+\left(1-\chi_{(E)_{\infty}}\right) f_{i} .
$$


Thus, since $\chi_{E} d \mu_{n} \stackrel{*}{\rightarrow} \chi_{E_{\infty}} d \mu_{\infty}$ and since $\mu_{\infty}$ gives measure zero to every sphere due to the geodesic property and doubling of $X_{\infty}$ (recall (5.2)), we get

$$
\begin{aligned}
\lim _{n \rightarrow \infty} & \int_{B_{n}\left(x_{n},[1+\tau] R\right) \backslash B_{n}\left(x_{n}, R\right)}\left|f_{i, n}-\chi_{E}\right| d \mu_{n} \\
& =\lim _{n \rightarrow \infty} \int_{B_{n}\left(x_{n},[1+\tau] R\right) \backslash B_{n}\left(x_{n}, R\right)}\left[\left(1-f_{i, n}\right) \chi_{E}+\left(1-\chi_{E}\right) f_{i, n}\right] d \mu_{n} \\
& =\int_{B_{X_{\infty}}(z,[1+\tau] R) \backslash B_{X_{\infty}}(z, R)}\left[\left(1-f_{i}\right) \chi_{(E)_{\infty}}+\left(1-\chi_{(E)_{\infty}}\right) f_{i}\right] d \mu_{\infty} \\
& =\int_{B_{X_{\infty}}(z,[1+\tau] R) \backslash B_{X_{\infty}}(z, R)}\left|f_{i}-\chi_{(E)_{\infty}}\right| d \mu_{\infty} .
\end{aligned}
$$

Now letting $n \rightarrow \infty$ and using (6.14), we obtain

$$
\begin{aligned}
\pi_{\infty}\left(B_{X_{\infty}}\left(x_{\infty}, \rho\right)\right) \leq & \pi_{\infty}\left(B_{X_{\infty}}\left(x_{\infty}, \rho\right) \backslash B_{X_{\infty}}(z, R)\right)+\int_{B_{X_{\infty}}(z,[1+\tau] R)} v_{i} d \mu_{\infty} \\
& +\frac{2}{\tau R} \int_{B_{X_{\infty}}(z,[1+\tau] R) \backslash B_{X_{\infty}}(z, R)}\left|f_{i}-\chi_{(E)_{\infty}}\right| d \mu_{\infty} .
\end{aligned}
$$

Thus we get

$$
\pi_{\infty}\left(B_{X_{\infty}}(z, R)\right) \leq \int_{B_{X_{\infty}}(z,[1+\tau] R)} v_{i} d \mu_{\infty}+\frac{2}{\tau R} \int_{B_{X_{\infty}}(z,[1+\tau] R) \backslash B_{X_{\infty}}(z, R)}\left|f_{i}-\chi_{(E)_{\infty}}\right| d \mu_{\infty} .
$$

Now letting $i \rightarrow \infty$ gives

$$
\pi_{\infty}\left(B_{X_{\infty}}(z, R)\right) \leq V\left(\chi_{(E)_{\infty}}+\varphi, B_{X_{\infty}}(x,[1+2 \tau] R)\right)
$$

where we used the fact that $f_{i} \rightarrow \chi_{(E)_{\infty}}$ in $L_{\mathrm{loc}}^{1}\left(X_{\infty}\right)$ and $v_{i} d \mu_{\infty} \stackrel{*}{\rightarrow} d V\left(\chi_{(E)_{\infty}}+\varphi, \cdot\right)$. Now letting $\tau \rightarrow 0$ and finally using the assumption $P\left((E)_{\infty}, \partial B_{X_{\infty}}(z, R)\right)=0$ we obtain

$$
\pi_{\infty}\left(B_{X_{\infty}}(z, R)\right) \leq V\left(\chi_{(E)_{\infty}}+\varphi, B_{X_{\infty}}(z, R)\right) .
$$

Now by Theorem 5.5 we have

$$
P\left((E)_{\infty}, B_{X_{\infty}}(z, R)\right) \leq C V\left(\chi_{(E)_{\infty}}+\varphi, B_{X_{\infty}}(z, R)\right)
$$

where $C$ is the comparison constant that connects $\pi_{\infty}$ to $P\left((E)_{\infty}, \cdot\right)$. Thus choosing $K=C$ yields the desired outcome. This completes the proof.

6.3. Concluding remarks. In Section 4 we have shown that any asymptotic limit, at $\mu$-almost every point, of a BV function is a function of least gradient on a corresponding tangent space $X_{\infty}$ and is Lipschitz continuous with a constant minimal $p$-weak upper gradient. In Section 6 we have shown that given a set $E$ of finite perimeter in $X$, at $\mathcal{H}$-almost every point of its measure-theoretic boundary we have the existence of an asymptotic limit set $(E)_{\infty} \subset X_{\infty}$ such that this asymptotic limit set is of quasiminimal boundary surface (that is, $\chi_{(E)_{\infty}}$ is of quasi-least gradient).

Remark 6.12. If $u \in B V(X)$, from the co-area formula we know that for almost every $t \in \mathbb{R}$ its super-level set

$$
E_{t}:=\{x \in X: u(x)>t\}
$$

is of finite perimeter in $X$. Let $\mathbb{R}_{F}$ be the collection of all $t \in \mathbb{R}$ for which $E_{t}$ is of finite perimeter, and let $A \subset \mathbb{R}_{F}$ be a countable dense subset of $\mathbb{R}_{F}$. For each $t \in A$ let $K_{t}$ be the collection of all points in $X$ at which the conclusion of Theorem 6.3 fails for $E_{t}$; then $\mathcal{H}\left(\bigcup_{t \in A} K_{t}\right)=0$. Let 
$x \in S_{u} \backslash \bigcup_{t \in A} K_{t}$, where $S_{u}$ is the jump set of $u$. Note that if $x \in X \backslash \partial^{*} E_{t}$, then for every tangent space $X_{\infty}$ based at that point, the corresponding set $\left(E_{t}\right)_{\infty}$ is either all of $X_{\infty}$ or is empty, and hence does satisfy the conclusion of Theorem 6.3. Thus we have here that $K_{t} \subset \partial^{*} E_{t}$. A Cantor diagonalization argument gives us for each $t \in A$ an asymptotic limit $\left(E_{t}\right)_{\infty} \subset X_{\infty}$, with $X_{\infty}$ a tangent space to $X$ based at $x$, of the set $E_{t}$. We know then that each $\left(E_{t}\right)_{\infty}$ is of quasiminimal boundary in $X_{\infty}$ in the sense of [25], with the quasiminimality constant $K$ independent of $t$. Moreover, note that if $t_{1}, t_{2} \in A$ such that $t_{1}<t_{2}$, then $E_{t_{2}} \subset E_{t_{1}}$ and so by the construction of $\left(E_{t}\right)_{\infty}$ we have that $\left(E_{t_{2}}\right)_{\infty} \subset\left(E_{t_{1}}\right)_{\infty}$. Indeed, by the definition of $(E)_{\infty}$ from the discussion before Lemma 5.1, we have that when $z \in X_{\infty}$ for which $z \in\left(E_{t_{2}}\right)_{\infty}$, we have $\mu_{\infty}^{E_{t_{2}}} \leq \mu_{\infty}^{E_{t_{1}}}$ on $X_{\infty}$ (because $E_{t_{2}} \subset E_{t_{1}} \subset X$ ), and so

$$
1=\lim _{r \rightarrow 0^{+}} \frac{\mu_{\infty}^{E_{t_{2}}}\left(B_{X_{\infty}}(z, r)\right)}{\mu_{\infty}\left(B_{X_{\infty}}(z, r)\right)} \leq \lim _{r \rightarrow 0^{+}} \frac{\mu_{\infty}^{E_{t_{1}}}\left(B_{X_{\infty}}(z, r)\right)}{\mu_{\infty}\left(B_{X_{\infty}}(z, r)\right)} \leq 1
$$

and so we must have $z \in\left(E_{t_{1}}\right)_{\infty}$. In this discussion, recall that we have fixed $x \in S_{u} \backslash \bigcup_{t \in A} K_{t}$. We can now set

$$
u_{\infty}(z):=\sup \left\{t \in A: z \in\left(E_{t}\right)_{\infty}\right\} .
$$

An argument as in the proof of [29, Theorem 4.10] tells us that $u_{\infty}$ is of quasi-least gradient in $X_{\infty}$. It would be interesting to know in which sense, if any, is this $u_{\infty}$ an asymptotic limit of $u$ at $x$.

The limit set $E_{\infty}$ is a quasi-minimizer according to Theorem 6.3 , in contrast to the minimizer property of the limit $u_{\infty}$ of $u$ at the absolutely continuous point of $\|D u\|$, see Theorem 4.9. The next example shows that this disconnect is real and is not an artifact of our proof.

Example 6.13. For positive integers $n$ let $a_{n}=1 / n$ ! and $b_{n}=-a_{n}$. Let $X=\mathbb{R}$ be equipped with the Euclidean metric and with a weighted measure $d \mu=w d \mathcal{L}^{1}$. Let us choose the weight $w$ so that

$$
w(x)= \begin{cases}2 & \text { if } b_{2 n-1}<x \leq b_{2 n} \text { or } a_{2 n} \leq x<a_{2 n-1} \\ 1 & \text { otherwise }\end{cases}
$$

Then if we choose the base point $x=0$ and the sequence of scales $r_{n}=1 /(2 n-1)$ !, we can see that the limit space $X_{\infty}=\mathbb{R}$ is equipped with the measure $\mu_{\infty}$ given by $d \mu_{\infty}=\left(\chi_{[-1,1]}+\frac{1}{2} \chi_{\mathbb{R} \backslash[-1,1]}\right) d \mathcal{L}^{1}$. If we take $E=(-\infty, 0]$, then $E$ is of finite perimeter with perimeter measure $P(E, \cdot)$ the Dirac measure supported at 0 . The limit set $E_{\infty}=(-\infty, 0)$ is quasiminimal, but is not a minimal set as $F:=(-\infty, 1)$ has a smaller perimeter measure; here, the perimeter measure $P_{\infty}\left(E_{\infty}, X_{\infty}\right)=1$ whereas $P_{\infty}\left(F, X_{\infty}\right)=\frac{1}{2}$, and note that $E_{\infty} \Delta F$ is a relatively compact subset of $X_{\infty}$.

Remark 6.14. In Definition 6.2 of quasiminimality we used balls $B(x, R)$. The study undertaken in [25] is applicable to functions satisfying this definition; however, the notion of quasiminimality given in [25] is slightly stronger, namely whenever $\varphi$ is a compactly supported $B V$ function on $X$, we have

$$
V(u, \operatorname{supp}(\varphi)) \leq K V(u+\varphi, \operatorname{supp}(\varphi))
$$

The proof given in Subsection 6.2 can be easily adapted to prove that $\chi_{(E)_{\infty}}$ satisfies this stronger version, but the proof gets messy, and hence we gave the relatively more transparent proof showing that $\chi_{(E)_{\infty}}$ satisfies Definition 6.2. To prove the stronger quasiminimality criterion of [25], one first modifies the stitching lemma (Lemma 6.11) by replacing $B(x, a), B(x, b)$ with open sets $U, V$ with $U \Subset V$ and considering $\eta$ to be a Lipschitz function with $\eta=1$ on $U, \eta=0$ on $X \backslash V$. The term $2 /(b-a)$ is then replaced with a constant $C$ that depends solely on $U, V$. Next, in the proof of quasiminimality, one replaces $B_{X_{\infty}}(z,[1+\tau] R)$ with $U_{\tau}$ where $U$ is the support of $\varphi$ 
and $U_{\tau}=\left\{y \in X_{\infty}: d_{X_{\infty}}(y, U)<\tau\right\}$. In this case, $B_{n}\left(x_{n},[1+\tau] R\right)$ is replaced with a suitable approximation of $U_{\tau}$ in $X_{n}$, ensuring that this approximating open set is contained within $B_{n}(x, \rho)$ where $\rho=2\left[\operatorname{diam}_{X_{\infty}}(U)+\operatorname{dist}\left(U, x_{\infty}\right)\right]$.

Remark 6.15. While we have assumed throughout this paper that $X$ is a geodesic space, we can omit this additional assumption and assume a weaker 1-Poincaré inequality where the ball $B$ on the right-hand side of (2.4) is replaced by a concentric ball $\lambda B$ with radius $\lambda$ times the radius of $B$, see [21, Section 8.1]. The reason for this is as follows: a weak 1-Poincaré inequality implies that the space is quasiconvex (that is, every pair of points $x, y \in X$ can be joined by a rectifiable curve of length at most $C d(x, y)$ with $C$ depending solely on the doubling and Poincaré constants), and then a bi-Lipschitz change in the metric will allow the space to become geodesic. In geodesic spaces, a weak Poincaré inequality can be promoted to be a strong Poincaré inequality, that is, $\lambda=1$. This is discussed in [21, Theorem 9.1.15] and in [19]. The class of functions of bounded variation is invariant under a bi-Lipschitz metric change. Thus the assumptions of geodesicity and the strong version of the Poincaré inequality are not restrictions, only conveniences. This bi-Lipschitz change in the metric on $X$ would induce a bi-Lipschitz change in the tangent space $X_{\infty}$, with a bi-Lipschitz equivalent geodesic limit metric on $X_{\infty}$ obtained as a limit of re-scaled geodesics metrics on $X$. We obtain that the asymptotic limit function $u_{\infty}$ as in Theorem 4.9 is of least gradient with respect to this length metric on $X_{\infty}$, and therefore is of quasi-least gradient with respect to the original metric on the tangent space $X_{\infty}$.

\section{REFERENCES}

[1] L. Ambrosio, Fine properties of sets of finite perimeter in doubling metric measure spaces, Calculus of variations, nonsmooth analysis and related topics. Set-Valued Anal. 10 (2002), 111-128.

[2] L. Ambrosio and S. Di Marino, Equivalent definitions of BV space and of total variation on metric measure spaces, J. Funct. Anal. 266 (2014), 4150-4188.

[3] L. Ambrosio, N. Fusco, and D. Pallara, Functions of bounded variation and free discontinuity problems. Oxford Mathematical Monographs. The Clarendon Press, Oxford University Press, New York, 2000.

[4] L. Ambrosio, R. Ghezzi, and V. Magnani, BV functions and sets of finite perimeter in sub-Riemannian manifolds, Ann. Inst. H. Poincaré Anal. Non Linéaire 32 (2015), 489-517.

[5] L. Ambrosio, M. Miranda, Jr., and D. Pallara, Special functions of bounded variation in doubling metric measure spaces, Calculus of variations: topics from the mathematical heritage of E. De Giorgi, 1-45, Quad. Mat., 14, Dept. Math., Seconda Univ. Napoli, Caserta, 2004.

[6] L. Ambrosio and P. Tilli, Topics on analysis in metric spaces, Oxford Lecture Series in Mathematics and its Applications, 25. Oxford University Press, Oxford, 2004.

[7] A. Bellaïche, The tangent space in sub-Riemannian geometry, In Bellaïche A., Risler JJ. (eds), Sub-Riemannian Geometry, Progress in Mathematics 144 Birkhäuser Basel (1996). https://doi.org/10.1007/978-3-0348-9210-0_1

[8] A. Björn and J. Björn, Nonlinear potential theory on metric spaces, EMS Tracts in Mathematics, 17. European Mathematical Society (EMS), Zürich, 2011.

[9] S. Buckley, Is the maximal function of a Lipschitz function continuous?, Ann. Acad. Sci. Fenn. Math. 24 (1999), 519-528.

[10] D. Burago, Y. Burago, and S. Ivanov, A course in metric geometry, Graduate Studies in Mathematics 33, American Mathematical Society, Providence, RI, 2001. http://www.math.psu.edu/petrunin/papers/akp-papers/bbi.pdf

[11] J. Cheeger, Differentiability of Lipschitz functions on metric measure spaces, Geom. Funct. Anal. 9 (1999), 428-517.

[12] L. C. Evans, R. F. Gariepy, Measure Theory and Fine Properties of Functions, Studies in Advanced Mathematics 5, CRC Press, 1992.

[13] B. Franchi, R. Serapioni, and F. Serra-Cassano, Rectifiability and perimeter in the Heisenberg group, Math. Ann. 321 (2001), 479-531. 
[14] B. Franchi, R. Serapioni, and F. Serra-Cassano, On the structure of finite perimeter sets in step 2 Carnot groups, J. Geom. Anal. 13 (2003), 421-466.

[15] B. Franchi, R. Serapioni, and F. Serra-Cassano, Regular hypersurfaces, intrinsic perimeter and implicit function theorem in Carnot groups, Comm. Anal. Geom. 11 (2003), 909-944.

[16] M. Gromov, Groups of polynomial growth and expanding maps. Publ. Math. I.H.É.S. 53 (1981), 53-78.

[17] H. Hakkarainen, J. Kinnunen, P. Lahti, and P. Lehtelä, Relaxation and integral representation for functionals of linear growth on metric measure spaces Anal. Geom. Metr. Spaces 4 (2016), 288-313.

[18] H. Hakkarainen, R. Korte, P. Lahti, and N. Shanmugalingam, Stability and continuity of functions of least gradient, Analysis and Geometry in Metric Spaces 3 (2015), 123-139.

[19] J. Heinonen, Lectures on Analysis on Metric Spaces, Universitext, Springer, 2001.

[20] J. Heinonen and P. Koskela, Quasiconformal maps in metric spaces with controlled geometry, Acta Math. 181 (1998) 1-61.

[21] J. Heinonen, P. Koskela, N. Shanmugalingam, and J. Tyson, Sobolev spaces on metric measure spaces: an approach based on upper gradients, New Mathematical Monographs 27, Cambridge University Press, 2015.

[22] D. Herron, Gromov-Hausdorff distance for pointed metric spaces, J. Anal. 24 (2016), 1-38.

[23] S. Keith, A differentiable structure for metric measure spaces, Adv. Math. 183 (2004), 271-315.

[24] S. Keith, Modulus and the Poincaré inequality on metric measure spaces, Math. Z., 245 (2003), 255-292.

[25] J. Kinnunen, R. Korte, A. Lorent, and N. Shanmugalingam, Regularity of sets with quasiminimal boundary surfaces in metric spaces, J. Geom. Anal. 23 (2013), 1607-1640.

[26] J. Kinnunen, R. Korte, N. Shanmugalingam, and H. Tuominen, Pointwise properties of functions of bounded variation in metric spaces, Rev. Mat. Complut. 27 (2014), 41-67.

[27] R. Korte and P. Lahti, Relative isoperimetric inequalities and sufficient conditions for finite perimeter on metric spaces, Ann. Inst. H. Poincaré Anal. Non Linéaire 31 (2014), 129-154.

[28] R. Korte, P. Lahti, X. Li, and N. Shanmugalingam, Notions of Dirichlet problem for functions of least gradient in metric measure spaces, Rev. Mat. Iberoam. 35 (2019), no. 6, 1603-1648.

[29] P. Lahti, L. Malý, N. Shanmugalingam, and G. Speight, Domains in metric measure spaces with boundary of positive mean curvature, and the Dirichlet problem for functions of least gradient, J. Geom. Anal. 29 (2019), no. 4, 3176-3220.

[30] E. LeDonne, A primer on Carnot groups: homogeneous groups, CC spaces, and regularity of their isometries, preprint, https://arxiv.org/pdf/1604.08579.pdf

[31] E. LeDonne, Metric spaces with unique tangents, Ann. Acad. Sci. Math. Fenn. 36 (2011), 683-694.

[32] V. Magnani, Elements of geometric measure theory on sub-Riemannian groups, Dissertation, Scuola Normale Superiore, Pisa, 2002.

[33] M. Miranda, Jr., Functions of bounded variation on "good" metric spaces, J. Math. Pures Appl. (9) 82 (2003), 975-1004.

[34] J. Mitchell, On Carnot-Carathéodory metrics, J. Differential Geom. 21 (1985), 35-45.

Addresses:

S.E.-B.: Research Unit of Mathematical Sciences, P.O.Box 3000, FI-90014 Oulu

E-mail: sylvester.eriksson-bique@oulu.fi

J.T.G.: Department of Mathematics and Statistics, Saint Louis University, Ritter Hall 307, 220 N. Grand Blvd., St. Louis, MO-63103, U.S.A.

E-mail: jim.gill@slu.edu

P.L.: Academy of Mathematics and Systems Science, Chinese Academy of Sciences, Beijing 100190, PR China. 
E-mail: panulahti@amss.ac.cn

N.S.: Department of Mathematical Sciences, P.O. Box 210025, University of Cincinnati, Cincinnati, OH 45221-0025, U.S.A.

E-mail: shanmun@uc.edu 